Supporting Information for

\title{
Spectroscopic and Computational Assessment of Silicon's Electrophilicity in Phosphinosilylium Cations
}

\author{
Kathleen M. Rileył, Andreas L. Wierschenł, Stephen J. Lee ${ }^{\ddagger}$, Michel R. Gagné ${ }^{\star}$ \\ ${ }^{\dagger}$ Caudill Laboratories, Department of Chemistry, University of North Carolina at Chapel Hill, \\ Chapel Hill, North Carolina, 27599-3290, United States \\ ‡U.S. Army Research Office, P.O. Box 12211, Research Triangle Park, North Carolina, 27709, \\ United States
}

\section{TABLE OF CONTENTS}

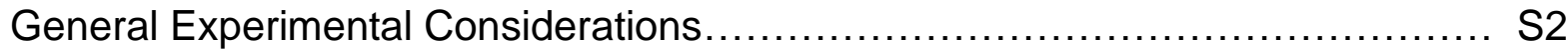

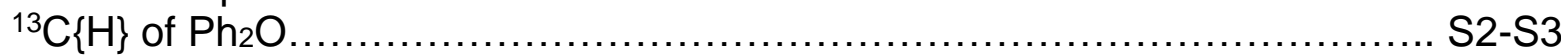

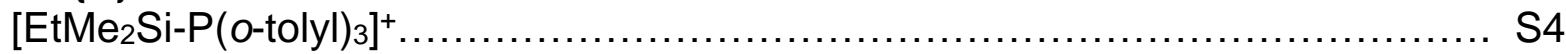

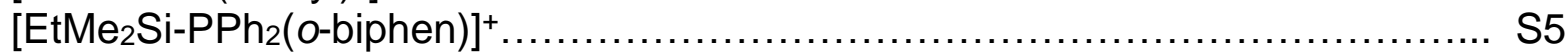

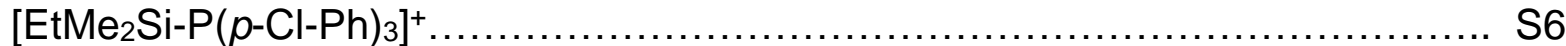

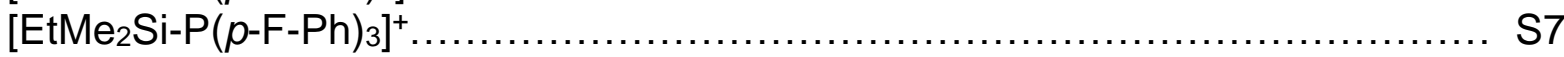

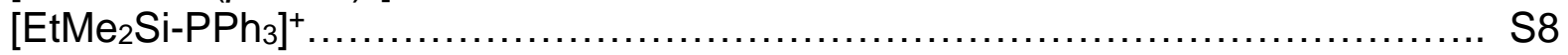

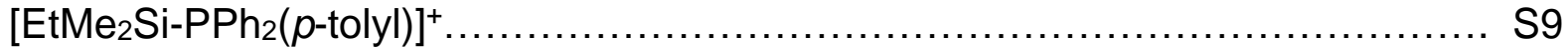

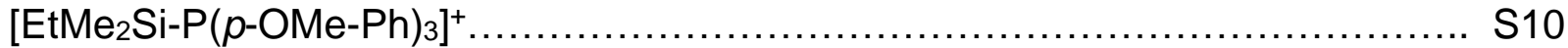

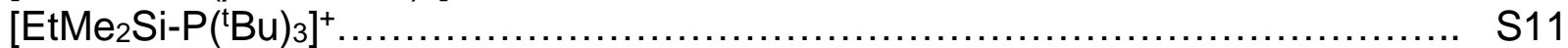

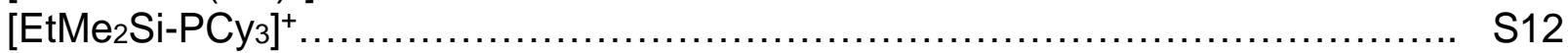

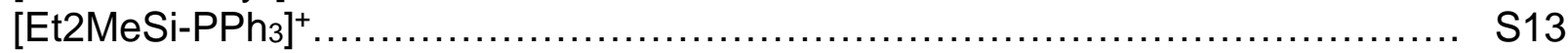

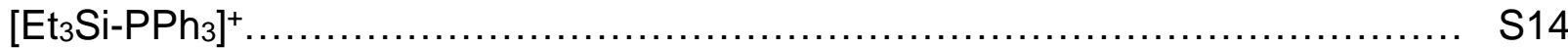

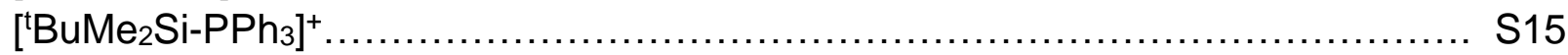

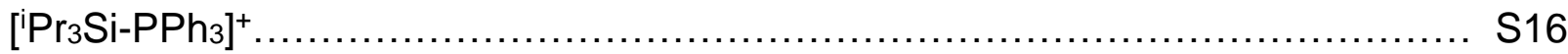

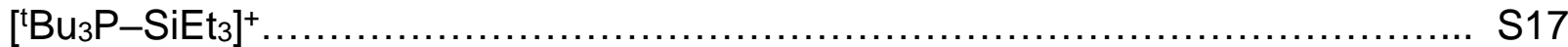

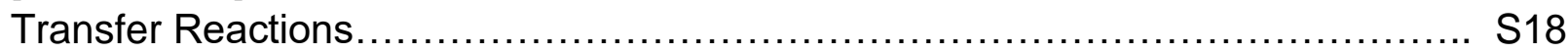

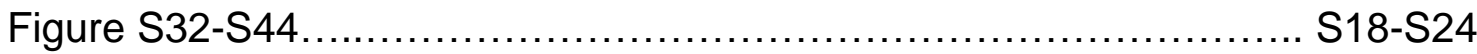

Silylicity Calculation Tables S1-S2 ....................................... S24-S25

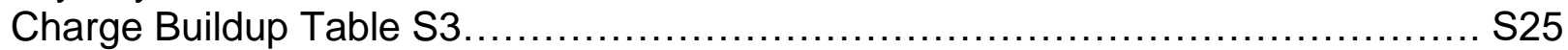

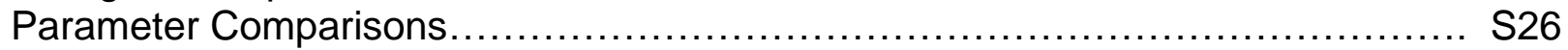

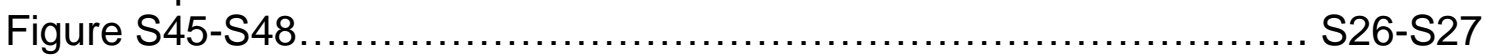

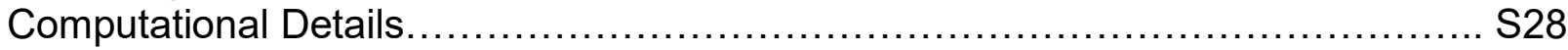

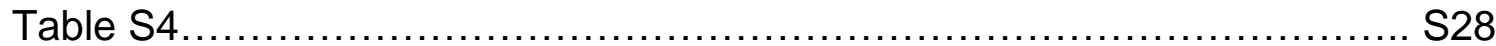

X-Ray Diffraction Experimental Data ....................................... S29-S42

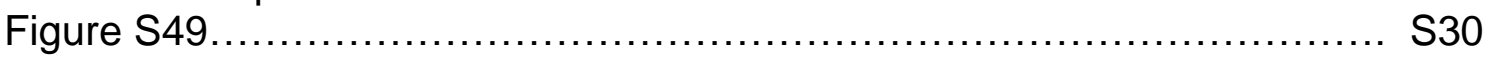

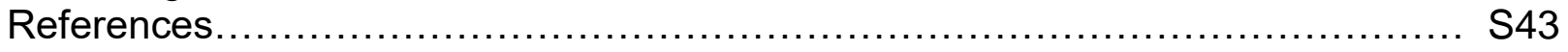


General Experimental Considerations. Phosphinosilylium syntheses were performed at room temperature $\left(22^{\circ} \mathrm{C}\right)$ in oven-dried $\left(130^{\circ} \mathrm{C}\right)$ NMR tubes in a nitrogen filled glovebox. Deuterated solvents were purchased from Cambridge Isotope Laboratories Inc. and degassed prior to use in the glovebox. The ortho-difluorobenzene (DFB) and diphenylether were dried by distillation over $\mathrm{CaH}_{2}$ and degassed prior to use in the box.

NMR spectra was obtained using a Bruker Avance 600,500 , or $400 \mathrm{MHz}$ or Bruker NEO 400 or $600 \mathrm{MHz}$ spectrometer at standard temperature and pressure. Chemical shifts were recorded as $\delta$ in ppm. ${ }^{1} \mathrm{H},{ }^{29} \mathrm{Si}-\mathrm{HMBC}$ NMR spectra were used to obtain ${ }^{29} \mathrm{Si}$ chemical shifts with tetramethylsilane in 1:5 DFB:Toluene- $d_{8}$ as an external reference. Residual deuterated solvent protons were used as reference in ${ }^{1} \mathrm{H}$ NMR while phosphoric acid was used as an external reference for ${ }^{31} \mathrm{P}$ NMR. Multiplicity is defined as follows: ssinglet, d-doublet, t-triplet, q-quartet, p-pentet, m-multiplet.

Synthesis of [EtMe2Si-PR $]^{+}$(1a-m). In a glovebox, diphenyl ether (1 eq) was added to a 1:5 difluorobenzene:Toluene-ds mixture. This mixture was added to $1 \mathrm{eq}$ of tritylB $\left(\mathrm{C}_{6} \mathrm{~F}_{5}\right)_{4}$ and mixed thoroughly. Ethyldimethylsilane $(1.1 \mathrm{eq})$ was then added to the solution until the solution went colorless. ${ }^{1}$ This solution was added to the appropriate phosphine and transferred to an oven-dried or flame-dried NMR tube. In each case, the aromatic ${ }^{1} \mathrm{H}$ peaks were obscured by the solvent.

Silylium Transfer Reactions. In a glovebox, the appropriate phosphinosilylium was generated, and dissolved in a 1:5 difluorobenzene:Toluene-d8 mixture $\left(\mathrm{R}_{3} \mathrm{Si}_{-} \mathrm{PR}_{3}{ }^{+}(\mathbf{1 a}-\mathbf{m})\right)$. After initial analysis, the NMR tube was returned to the glovebox. The appropriate phosphine for silylium transfer was dissolved in a 1:5 difluorobenzene:Toluene-d8 mixture, added to the NMR tube containing the phosphinosilylium, and allowed to react for 24 hours to reach equilibrium.

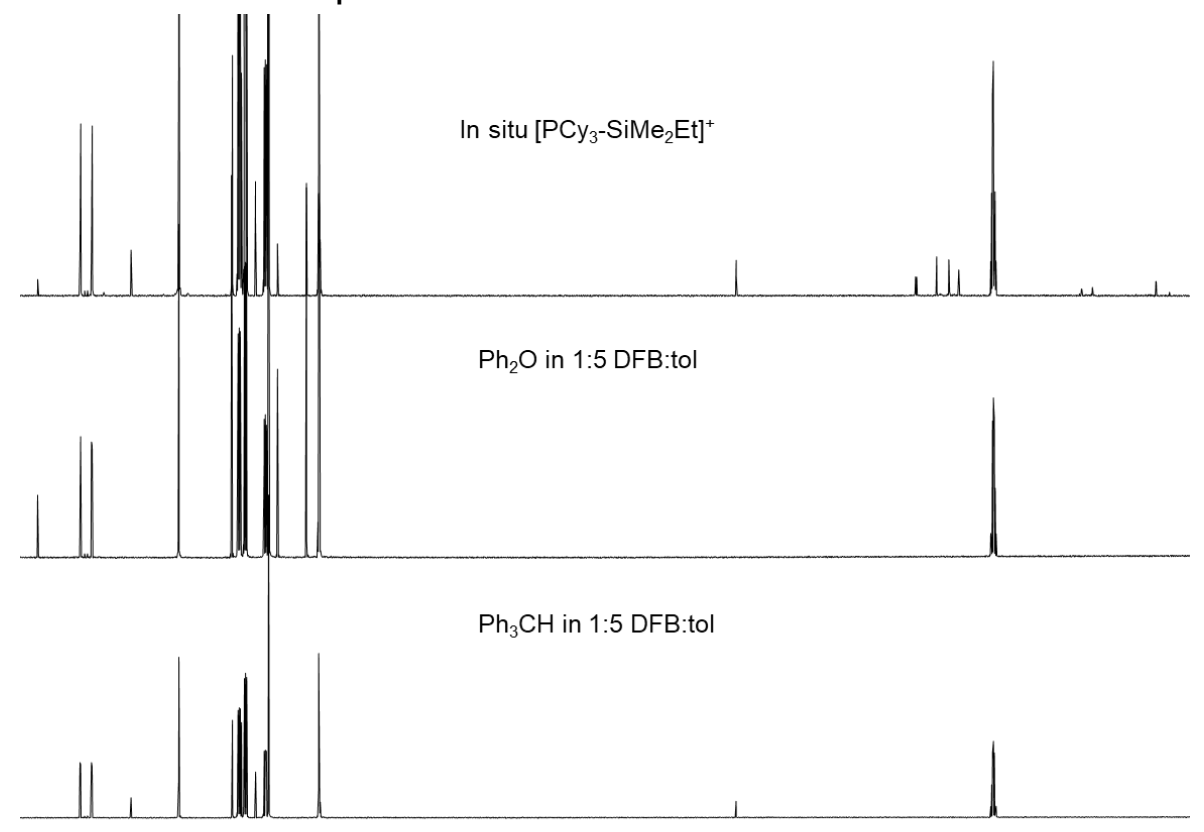

Figure $\mathrm{S} 1 .{ }^{13} \mathrm{C}\{\mathrm{H}\}$ NMR of in situ $\left[\mathrm{Cy}_{3} \mathrm{P}-\mathrm{SiMe}_{2} \mathrm{Et}\right]^{+}, \mathrm{Ph}{ }_{2} \mathrm{O}$, and $\mathrm{Ph}_{3} \mathrm{CH}$ in 1:5 DFB:toluene indicating no interaction of the two with in situ generation of phosphinosilyliums. 

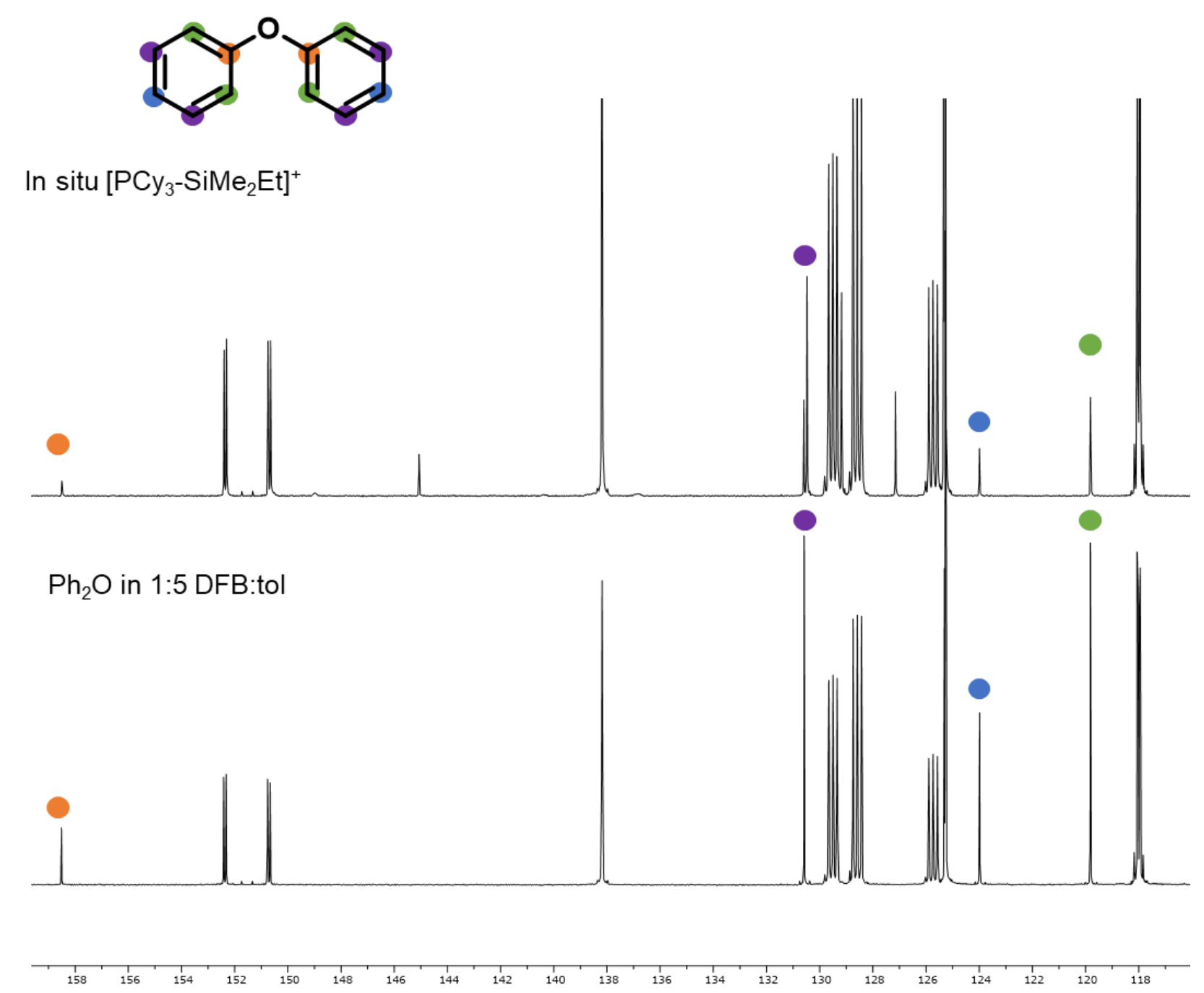

Figure S2. Labeled $\mathrm{Ph}_{2} \mathrm{O}$ in ${ }^{13} \mathrm{C}\{\mathrm{H}\} \mathrm{NMR}$ spectra where no chemical shift changes are observed indicating no interference after phosphine addition. 
[EtMe2Si-P(o-tolyl) $]^{+}\left[\mathrm{B}\left(\mathrm{C}_{6} \mathrm{~F}_{5}\right)_{4}\right]^{-}(1 \mathrm{a})$

${ }^{1} \mathrm{H}$ NMR $\left(600 \mathrm{MHz}, 1: 5 \mathrm{DFB}: T o l u e n e-d_{8}\right): \delta 1.86$ (s, $9 \mathrm{H}, \mathrm{CH}_{3}-\mathrm{Ar}$ ), 0.75 (td, ${ }^{3} J_{H H}=7.9$, $\left.{ }^{4} J_{P H}=1.9 \mathrm{~Hz}, 3 \mathrm{H}, \mathrm{Si}-\mathrm{CH}_{2} \mathrm{CH}_{3}\right), 0.58\left(\mathrm{qd},{ }^{3} J_{H H}=8.0,{ }^{3} J_{P H}=2.8 \mathrm{~Hz}, 2 \mathrm{H}, \mathrm{Si}-\mathrm{CH}_{2} \mathrm{CH}_{3}\right), 0.18$ $\left(\mathrm{d},{ }^{3} \mathrm{JPH}=8.0 \mathrm{~Hz}, 6 \mathrm{H}, \mathrm{Si}-\mathrm{CH}_{3}\right) .{ }^{31} \mathrm{P}\left\{{ }^{1} \mathrm{H}\right\} \mathrm{NMR}\left(243 \mathrm{MHz}, 1: 5 \mathrm{DFB}: T o l u e n e-d_{8}\right): \delta 2.5$ (s). ${ }^{29} \mathrm{Si} \mathrm{NMR}\left(99 \mathrm{MHz}, 1: 5 \mathrm{DFB}: T o l u e n e-d_{8}\right)$ : $\delta 21.8$ (s).

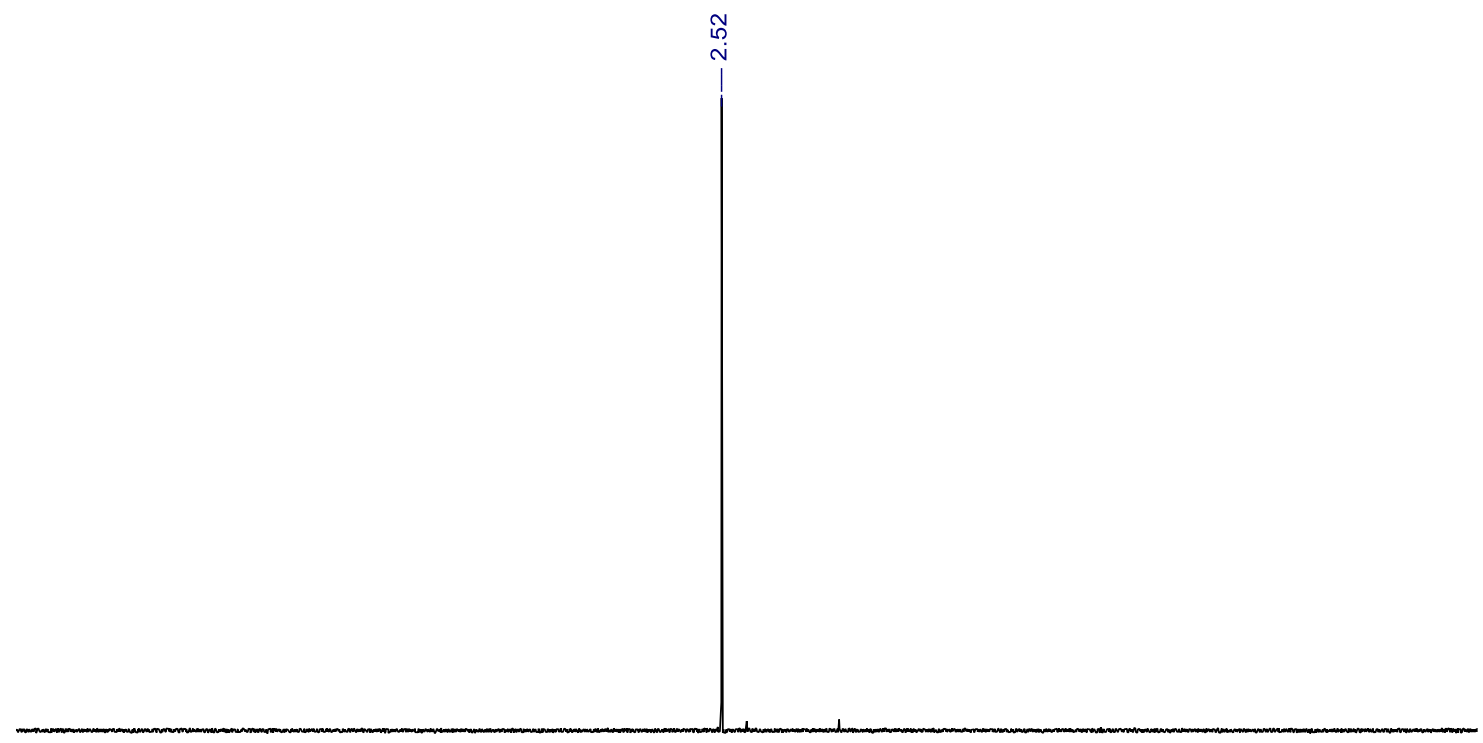

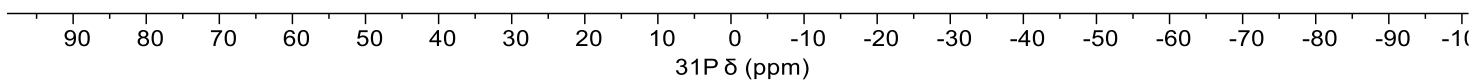

Figure S3. ${ }^{31} \mathrm{P}\{\mathrm{H}\}$ NMR spectrum of in situ $\left[\mathrm{EtMe} 2 \mathrm{Si}-\mathrm{P}(0 \text {-tolyl })_{3}\right]^{+}\left[\mathrm{B}\left(\mathrm{C}_{6} \mathrm{~F}_{5}\right)_{4}\right]^{-}$.

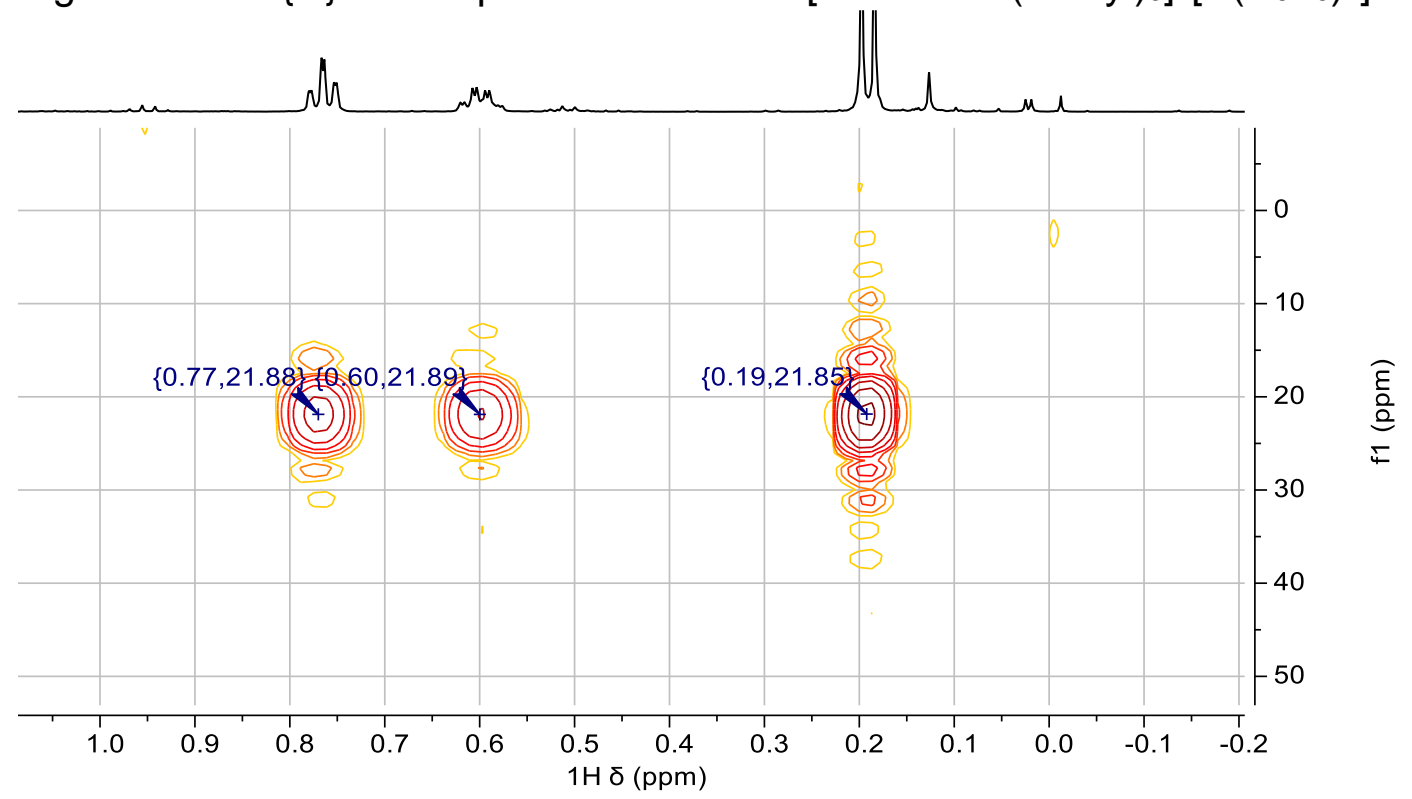

Figure S4. ${ }^{1} \mathrm{H},{ }^{29} \mathrm{Si}-\mathrm{HMBC}$ NMR spectrum of in situ [EtMer Si-P $(0$-tolyl $\left.) 3\right]^{+}\left[\mathrm{B}\left(\mathrm{C}_{6} \mathrm{~F}_{5}\right)_{4}\right]^{-}$. 
[EtMe2 Si-PPh 2 (o-biphen) $]^{+}\left[\mathrm{B}\left(\mathrm{C}_{6} \mathrm{~F}_{5}\right)_{4}\right]^{-}$(1b)

${ }^{1} \mathrm{H}$ NMR $\left(600 \mathrm{MHz}, 1: 5 \mathrm{DFB}: T o l u e n e-d_{8}\right): \delta 0.56\left(\mathrm{td},{ }^{3} J_{H H}=8.0,{ }^{4} J_{P H}=1.5 \mathrm{~Hz}, 3 \mathrm{H}, \mathrm{Si}-\right.$ $\left.\mathrm{CH}_{2} \mathrm{CH}_{3}\right), 0.27\left(\mathrm{qd},{ }^{3} \mathrm{~J}_{\mathrm{HH}}=7.9,{ }^{3} J_{P H}=3.6 \mathrm{~Hz}, 2 \mathrm{H}, \mathrm{Si}-\mathrm{CH}_{2} \mathrm{CH}_{3}\right),-0.16\left(\mathrm{~d},{ }^{3} \mathrm{~J}_{P H}=8.0 \mathrm{~Hz}\right.$, $\left.6 \mathrm{H}, \mathrm{Si}-\mathrm{CH}_{3}\right) .{ }^{31} \mathrm{P}\left\{{ }^{1} \mathrm{H}\right\}$ NMR $\left(243 \mathrm{MHz}, 1: 5 \mathrm{DFB}: T o l u e n e-d_{8}\right): \delta-0.86$ (s). ${ }^{29} \mathrm{Si}$ NMR $(99$ $\mathrm{MHz}, 1: 5$ DFB:Toluene- $\left.d_{8}\right): \delta 20.4(\mathrm{~s})$.

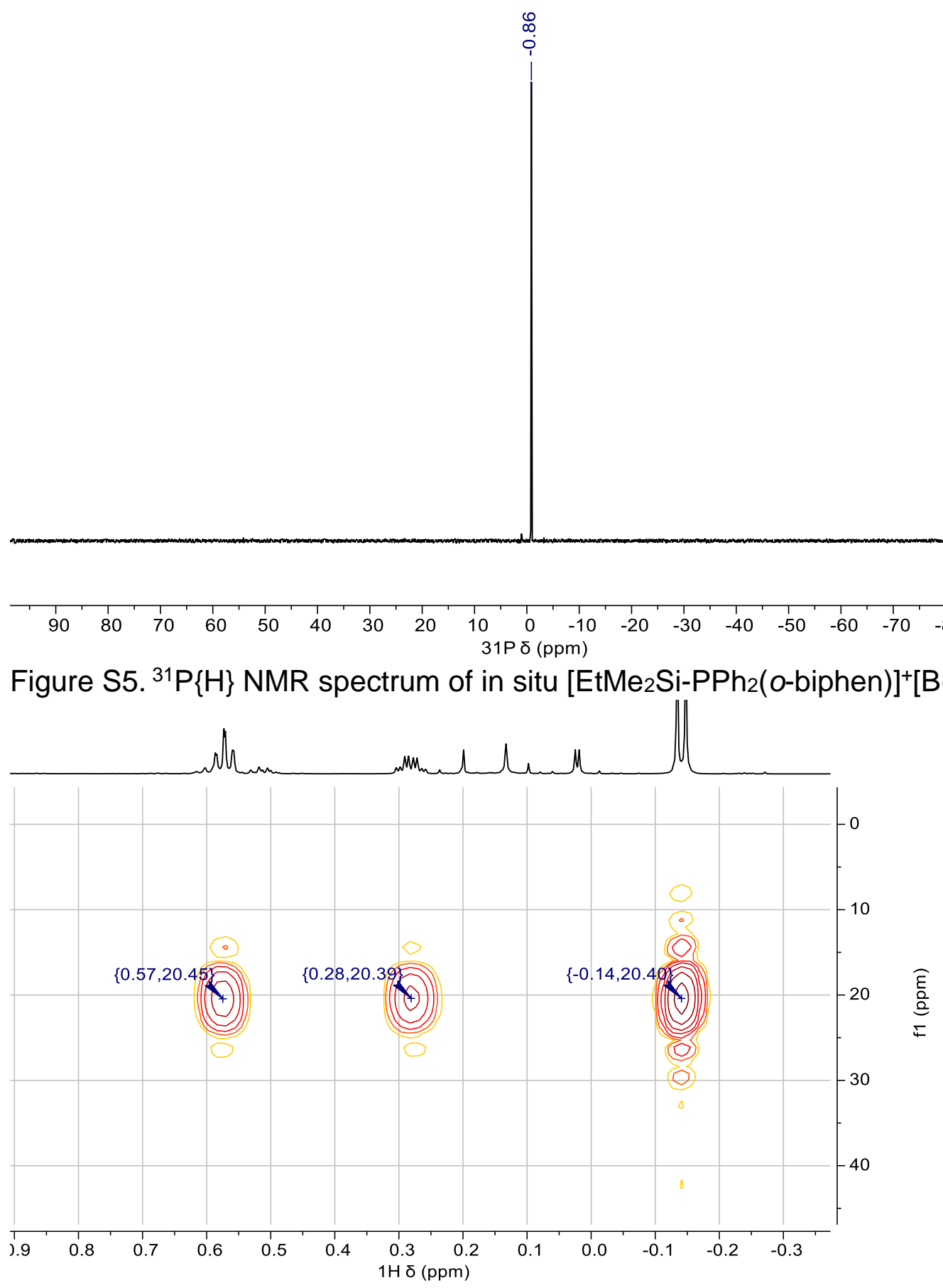

Figure S6. ${ }^{1} \mathrm{H},{ }^{29} \mathrm{Si}-\mathrm{HMBC}$ NMR spectrum of in situ [EtMe $2 \mathrm{Si}-\mathrm{PPh} 2(0-$ biphen $\left.)\right]^{+}\left[\mathrm{B}\left(\mathrm{C}_{6} \mathrm{~F}_{5}\right)_{4}\right]^{-}$. 
$\left[\mathrm{EtMe}{ }_{2} \mathrm{Si}-\mathrm{P}(\mathrm{p}-\mathrm{Cl}-\mathrm{Ph})_{3}\right]^{+}\left[\mathrm{B}\left(\mathrm{C}_{6} \mathrm{~F}_{5}\right)_{4}\right]^{-}(\mathbf{1 C})$

${ }^{1} \mathrm{H}$ NMR $\left(600 \mathrm{MHz}, 1: 5 \mathrm{DFB}: T o l u e n e-d_{8}\right): \delta 0.70\left(\mathrm{td},{ }^{3} \mathrm{~J}_{H H}=7.8,{ }^{4} \mathrm{~J}_{P H}=1.3 \mathrm{~Hz}, 3 \mathrm{H}, \mathrm{Si}-\right.$ $\left.\mathrm{CH}_{2} \mathrm{CH}_{3}\right), 0.59\left(\mathrm{~m}, 2 \mathrm{H}, \mathrm{Si}-\mathrm{CH}_{2} \mathrm{CH}_{3}\right), 0.17\left(\mathrm{~d},{ }^{3} J_{P H}=8.6 \mathrm{~Hz}, 6 \mathrm{H}, \mathrm{Si}_{-} \mathrm{CH}_{3}\right) \cdot{ }^{31} \mathrm{P}\left\{{ }^{1} \mathrm{H}\right\} \mathrm{NMR}$ (243 MHz, 1:5 DFB:Toluene- $d_{8}$ ): $\delta$-3.6 (s). $\left.{ }^{29} \mathrm{Si} \mathrm{NMR} \mathrm{(99} \mathrm{MHz,} \mathrm{1:5} \mathrm{DFB:Toluene-} d_{8}\right): \delta$ $18.3(\mathrm{~s})$.

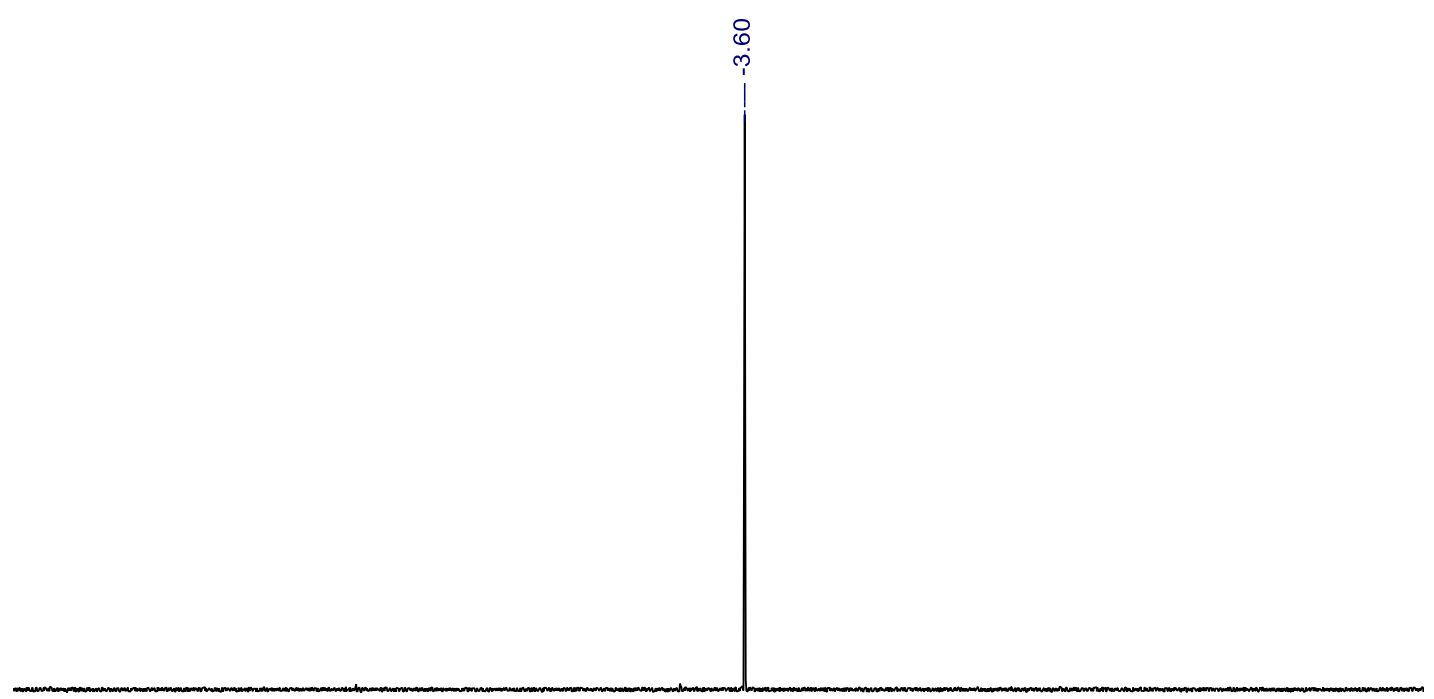

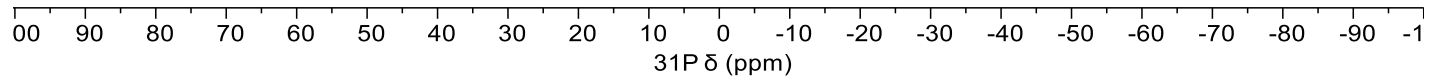

Figure $\mathrm{S} 7 .{ }^{31} \mathrm{P}\{\mathrm{H}\} \mathrm{NMR}$ spectrum of in situ $\left[\mathrm{EtMe} 2 \mathrm{Si}-\mathrm{P}(p-\mathrm{Cl}-\mathrm{Ph})_{3}\right]^{+}\left[\mathrm{B}\left(\mathrm{C}_{6} \mathrm{~F}_{5}\right)_{4}\right]^{-}$.

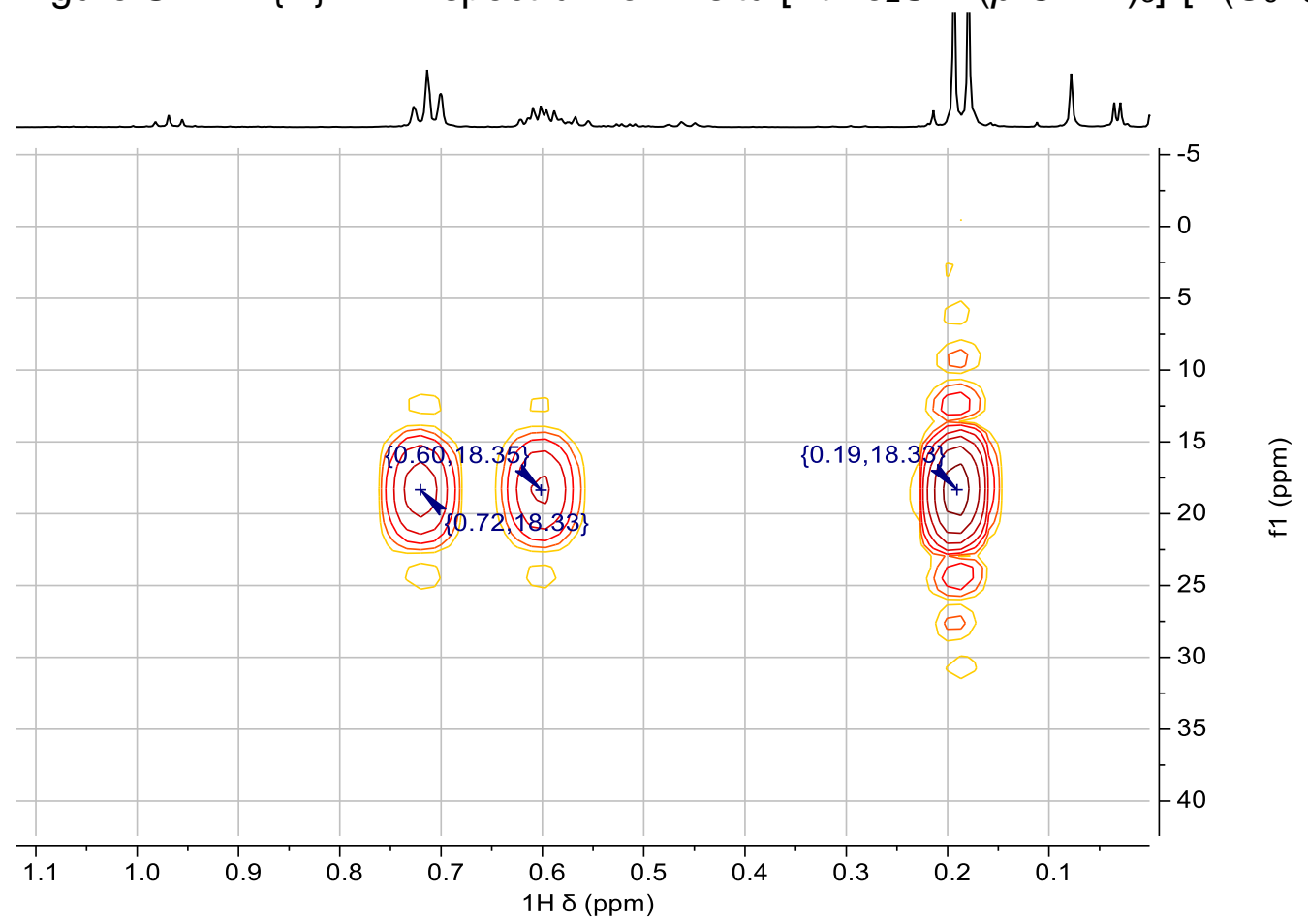

Figure S8. ${ }^{1} \mathrm{H},{ }^{29} \mathrm{Si}-\mathrm{HMBC}$ NMR spectrum of in situ $\left[\mathrm{EtMe} 2 \mathrm{Si}-\mathrm{P}(p-\mathrm{Cl}-\mathrm{Ph})_{3}\right]^{+}\left[\mathrm{B}\left(\mathrm{C}_{6} \mathrm{~F}_{5}\right)_{4}\right]^{-}$. 
$\left[\mathrm{EtMe}{ }_{2} \mathrm{Si}-\mathrm{P}(\boldsymbol{p}-\mathrm{F}-\mathrm{Ph})_{3}\right]^{+}\left[\mathrm{B}\left(\mathrm{C}_{6} \mathrm{~F}_{5}\right)_{4}\right]^{-}(\mathbf{1 d})$

${ }^{1} \mathrm{H}$ NMR $\left(600 \mathrm{MHz}, 1: 5 \mathrm{DFB}: T o l u e n e-d_{8}\right): \delta 0.70\left(\mathrm{td},{ }^{3} J_{H H}=7.8,{ }^{4} J_{P H}=1.3 \mathrm{~Hz}, 3 \mathrm{H}, \mathrm{Si}-\right.$ $\left.\mathrm{CH}_{2} \mathrm{CH}_{3}\right), 0.60\left(\mathrm{~m}, 2 \mathrm{H}, \mathrm{Si}-\mathrm{CH}_{2} \mathrm{CH}_{3}\right), 0.17\left(\mathrm{~d},{ }^{3}{ }_{\mathrm{PH}} \mathrm{h}=8.6 \mathrm{~Hz}, 6 \mathrm{H}, \mathrm{Si}-\mathrm{CH}_{3}\right) .{ }^{31} \mathrm{P}\left\{{ }^{1} \mathrm{H}\right\} \mathrm{NMR}$ (243 MHz, 1:5 DFB:Toluene- $d_{8}$ ): $\delta-4.1$ (s). ${ }^{29} \mathrm{Si} \mathrm{NMR} \mathrm{(99} \mathrm{MHz,} \mathrm{1:5} \mathrm{DFB:Toluene-} d_{8}$ ): $\delta$ 17.8 (s).

$\begin{array}{lllllllllllllllllllll}00 & 90 & 80 & 70 & 60 & 50 & 40 & 30 & 20 & \begin{array}{c}10 \\ 31 \mathrm{P} \delta(\mathrm{ppm})\end{array} & -20 & -30 & -40 & -50 & -60 & -70 & -80 & -90 & -11\end{array}$

Figure S9. ${ }^{31} \mathrm{P}\{\mathrm{H}\}$ NMR spectrum of in situ $\left[\mathrm{EtMe}_{2} \mathrm{Si}-\mathrm{P}(p-\mathrm{F}-\mathrm{Ph})_{3}\right]^{+}\left[\mathrm{B}\left(\mathrm{C}_{6} \mathrm{~F}_{5}\right)_{4}\right]^{-}$.

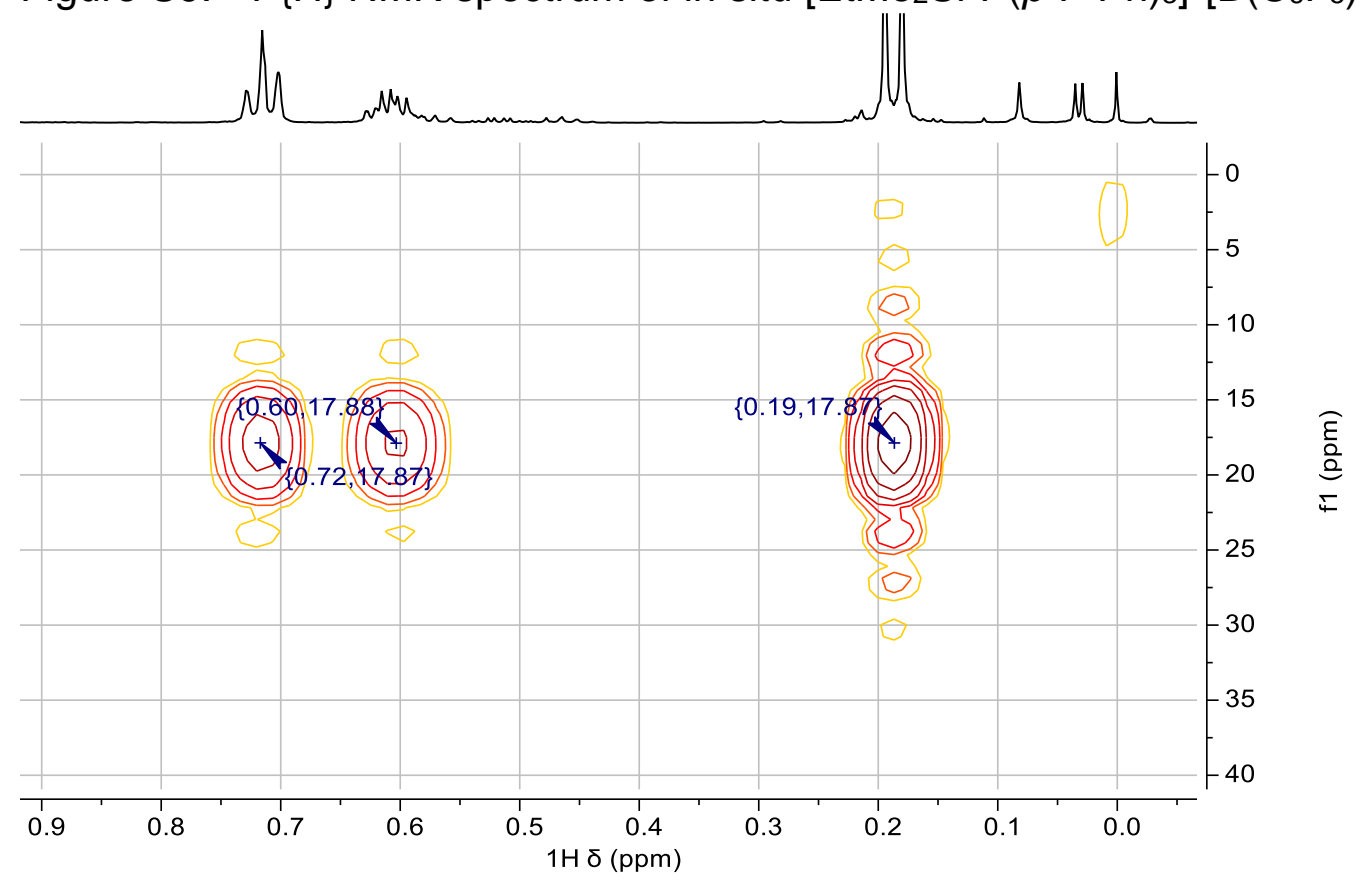

Figure $\mathrm{S} 10 .{ }^{1} \mathrm{H},{ }^{29} \mathrm{Si}-\mathrm{HMBC}$ NMR spectrum of in situ $\left[\mathrm{EtMe} 2 \mathrm{Si}-\mathrm{P}(p-\mathrm{F}-\mathrm{Ph})_{3}\right]^{+}\left[\mathrm{B}\left(\mathrm{C}_{6} \mathrm{~F}_{5}\right)_{4}\right]^{-}$. 
$\left[\mathrm{EtMe}_{2} \mathrm{Si}-\mathrm{PPh}_{3}\right]^{+}\left[\mathrm{B}\left(\mathrm{C}_{6} \mathrm{~F}_{5}\right)_{4}\right]^{-}(\mathbf{1 e})$

${ }^{1} \mathrm{H}$ NMR $\left(500 \mathrm{MHz}, 1: 5 \mathrm{DFB}:\right.$ Toluene- $\left.d_{8}\right): \delta 0.65\left(\mathrm{t},{ }^{3} \mathrm{~J}_{\mathrm{HH}}=7.4 \mathrm{~Hz}, 3 \mathrm{H}, \mathrm{Si}-\mathrm{CH}_{2} \mathrm{CH}_{3}\right), 0.58$ (t, $\left.{ }^{3} \mathrm{~J}_{\mathrm{HH}}=7.0 \mathrm{~Hz}, 2 \mathrm{H}, \mathrm{Si}-\mathrm{CH}_{2} \mathrm{CH}_{3}\right), 0.14\left(\mathrm{~s}, 6 \mathrm{H}, \mathrm{Si}-\mathrm{CH}_{3}\right) .{ }^{31} \mathrm{P}\left\{{ }^{1} \mathrm{H}\right\} \mathrm{NMR}(202 \mathrm{MHz}, 1: 5$ DFB:Toluene- $d_{8}$ ): $\delta-3.1$ (s). ${ }^{29} \mathrm{Si}$ NMR (99 MHz, 1:5 DFB:Toluene- $\left.d_{8}\right): \delta 16.4$ (s).

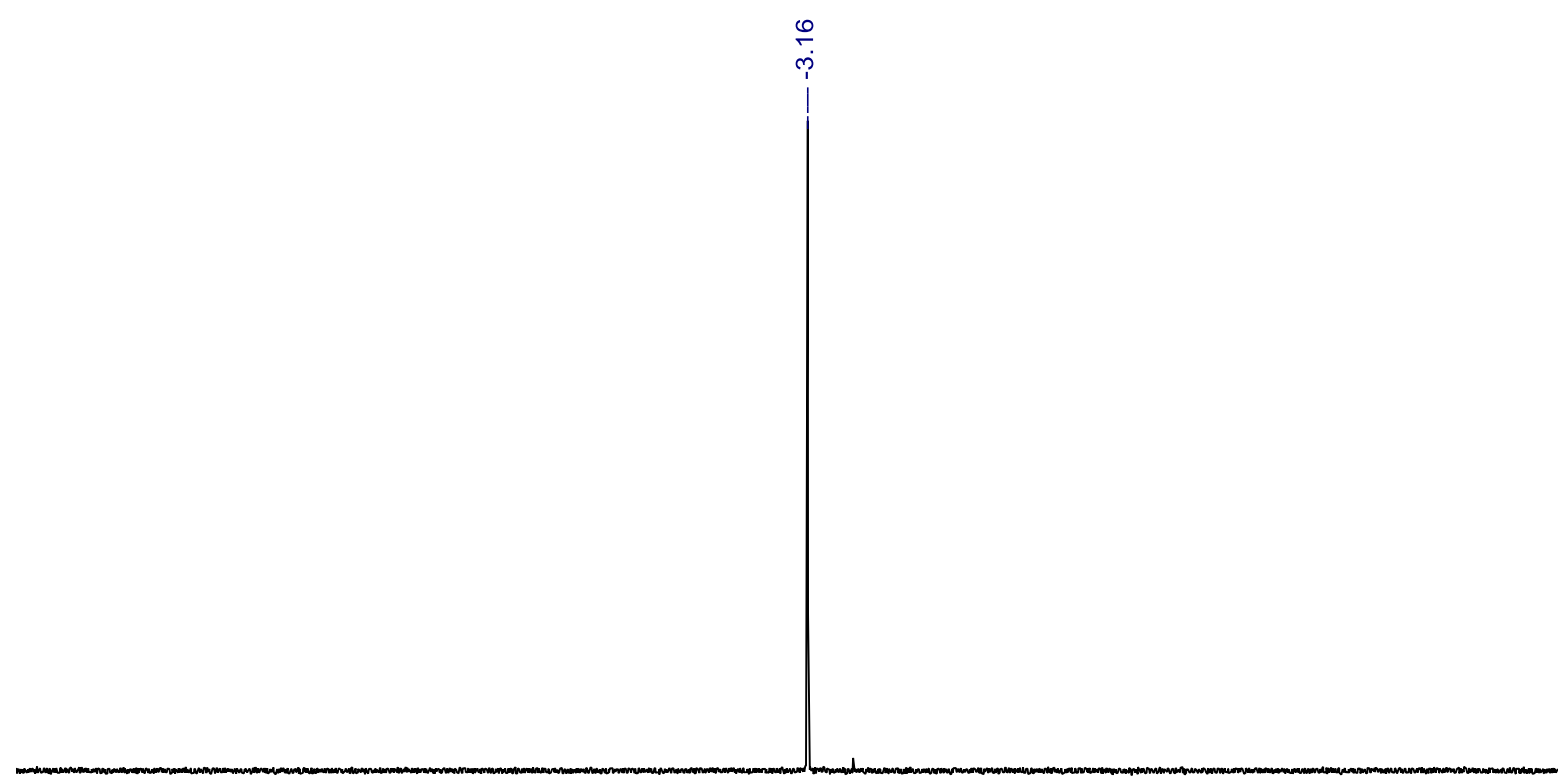

$\begin{array}{llllllllllllllllllll}90 & 80 & 70 & 60 & 50 & 40 & 30 & 20 & \begin{array}{c}10 \\ 31 \mathrm{P} \delta(\mathrm{ppm})\end{array} & -10 & -20 & -30 & -40 & -50 & -60 & -70 & -80 & -90 & -11\end{array}$

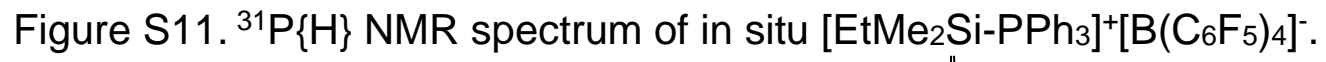

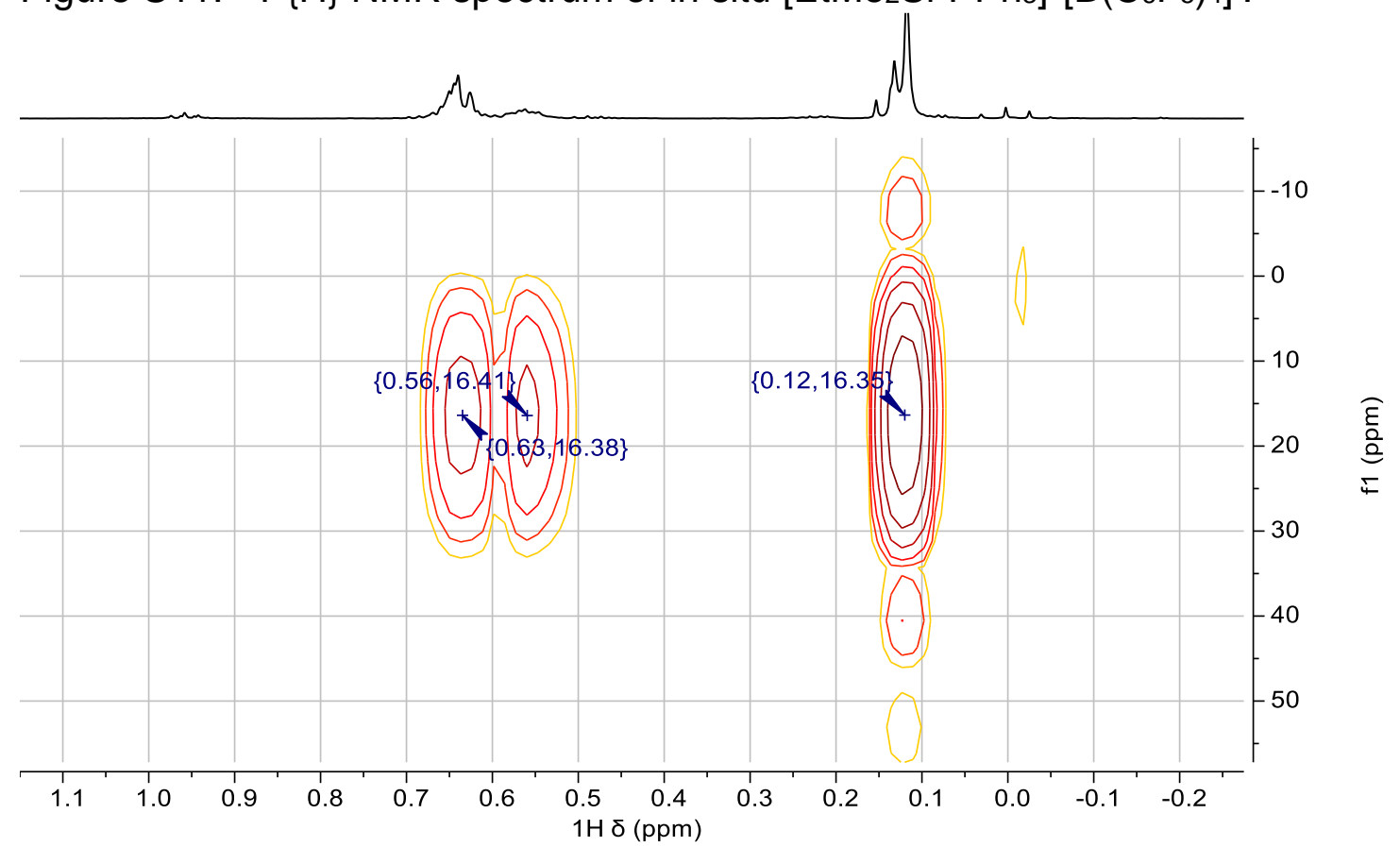

Figure $\mathrm{S} 12 .{ }^{1} \mathrm{H},{ }^{29} \mathrm{Si}-\mathrm{HMBC}$ NMR spectrum of in situ [EtMe2 $\left.\mathrm{Si}-\mathrm{PPh}_{3}\right]^{+}\left[\mathrm{B}\left(\mathrm{C}_{6} \mathrm{~F}_{5}\right)_{4}\right]^{-}$. 
[EtMe $\mathrm{Si}^{-P P h_{2}}(p \text {-tolyl) }]^{+}\left[\mathrm{B}\left(\mathrm{C}_{6} \mathrm{~F}_{5}\right)_{4}\right]^{-}(\mathbf{1 f})$

${ }^{1} \mathrm{H}$ NMR $\left(600 \mathrm{MHz}, 1: 5 \mathrm{DFB}: T o l u e n e-d_{8}\right): \delta 2.12$ (s, 3H, $\mathrm{CH}_{3}-\mathrm{Ar}$ ), 0.67 (td, ${ }^{3} J_{H H}=7.7$, $\left.{ }^{4} J_{P H}=1.4 \mathrm{~Hz}, 3 \mathrm{H}, \mathrm{Si}-\mathrm{CH}_{2} \mathrm{CH}_{3}\right), 0.61\left(\mathrm{~m}, 2 \mathrm{H}, \mathrm{Si}-\mathrm{CH}_{2} \mathrm{CH}_{3}\right), 0.17\left(\mathrm{~d},{ }^{3}{ }_{\mathrm{PH}}=8.2 \mathrm{~Hz}, 6 \mathrm{H}, \mathrm{Si}-\right.$ $\left.\mathrm{CH}_{3}\right) .{ }^{31} \mathrm{P}\left\{{ }^{1} \mathrm{H}\right\}$ NMR (243 MHz, 1:5 DFB:Toluene- $\left.d_{8}\right): \delta-3.5$ (s). ${ }^{29} \mathrm{Si} \mathrm{NMR}(99 \mathrm{MHz}, 1: 5$ DFB:Toluene- $\left.d_{8}\right): \delta 15.4(\mathrm{~s})$.

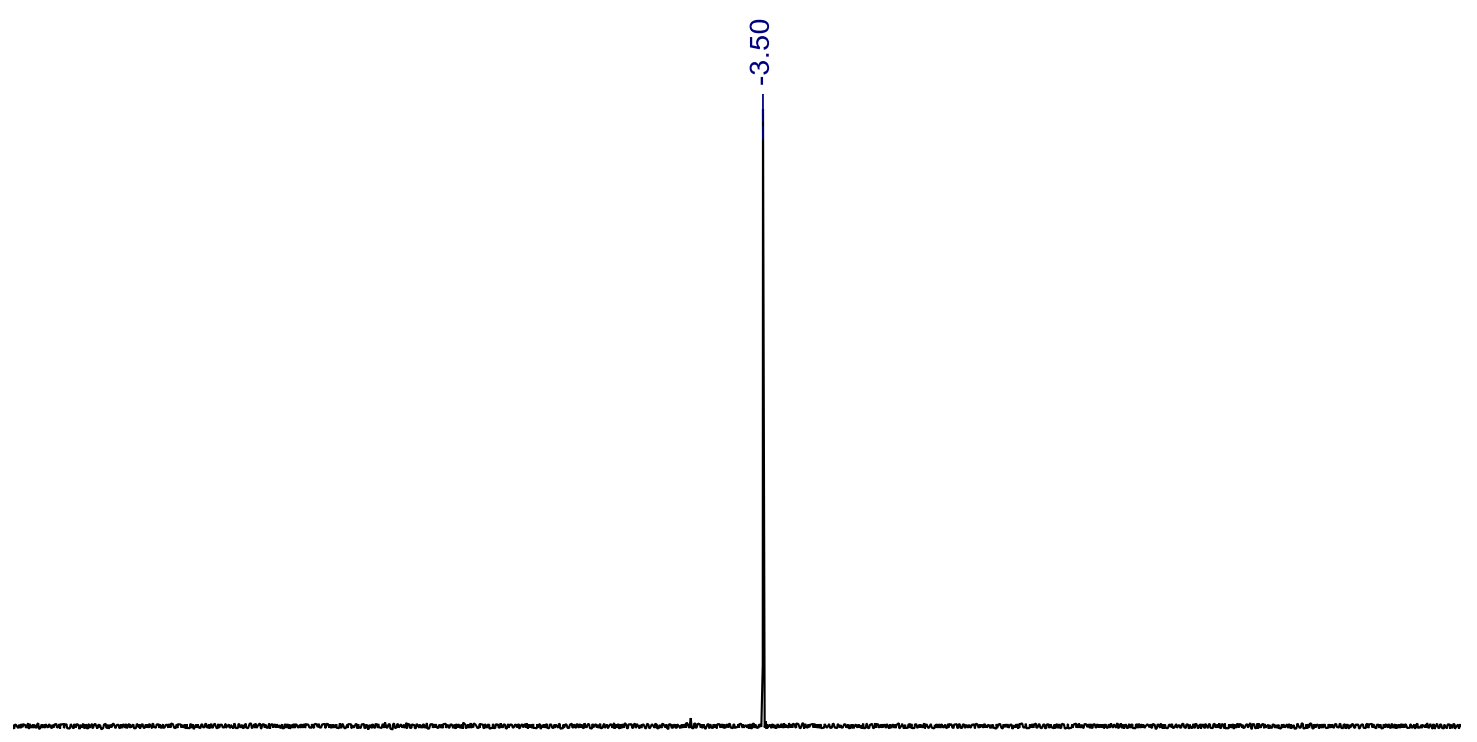

$\begin{array}{lllllllllllllllllllllllll}00 & 90 & 80 & 70 & 60 & 50 & 40 & 30 & 20 & 10 & 0 & -10 & -20 & -30 & -40 & -50 & -60 & -70 & -80 & -90 & -11 \\ 31 \mathrm{P} \delta(\mathrm{ppm}) & & & \end{array}$

Figure $\mathrm{S} 14 .{ }^{31} \mathrm{P}\{\mathrm{H}\}$ NMR spectrum of in situ [EtMe $2 \mathrm{Si}-\mathrm{PPh}_{2}(p$-tolyl $\left.)\right]^{+}\left[\mathrm{B}\left(\mathrm{C}_{6} \mathrm{~F}_{5}\right)_{4}\right]^{\text {. }}$.

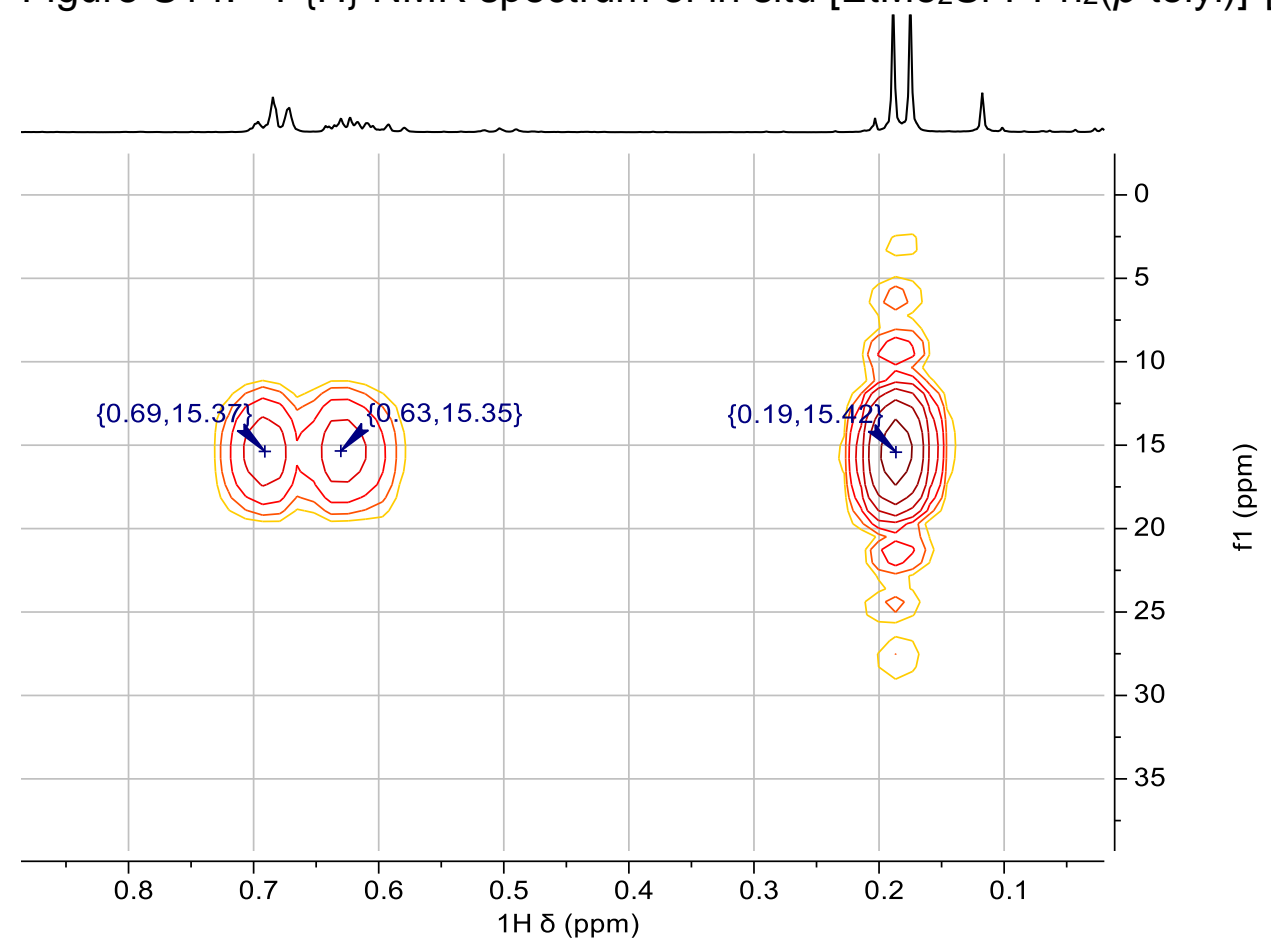

Figure $\mathrm{S} 15 .{ }^{1} \mathrm{H},{ }^{29} \mathrm{Si}-\mathrm{HMBC}$ NMR spectrum of in situ $\left[\mathrm{EtMe}_{2} \mathrm{Si}_{-} \mathrm{PPh}_{2}(p \text {-tolyl })\right]^{+}\left[\mathrm{B}\left(\mathrm{C}_{6} \mathrm{~F}_{5}\right) 4\right]^{-}$. 


\section{[EtMe $\left.{ }_{2} \mathrm{Si}-\mathrm{P}(\boldsymbol{p}-\mathrm{OMe}-\mathrm{Ph})_{3}\right]^{+}\left[\mathrm{B}\left(\mathrm{C}_{6} \mathrm{~F}_{5}\right)_{4}\right]^{-}(\mathbf{g})$}

${ }^{1} \mathrm{H}$ NMR (600 MHz, 1:5 DFB:Toluene-d8): $\delta 3.43\left(\mathrm{~s}, 9 \mathrm{H}, \mathrm{OCH}_{3}\right), 0.75(\mathrm{~m}, 3 \mathrm{H}$, Si$\left.\mathrm{CH}_{2} \mathrm{CH}_{3}\right), 0.69\left(\mathrm{~m}, 2 \mathrm{H}, \mathrm{Si}-\mathrm{CH}_{2} \mathrm{CH}_{3}\right), 0.24\left(\mathrm{~d},{ }^{3} \mathrm{~J}_{\mathrm{PH}}=8.1 \mathrm{~Hz}, 6 \mathrm{H}, \mathrm{Si}-\mathrm{CH}_{3}\right) .{ }^{31} \mathrm{P}\left\{{ }^{1} \mathrm{H}\right\} \mathrm{NMR}$ (243 MHz, 1:5 DFB:Toluene-d8): $\delta$-5.5 (s). ${ }^{29}$ Si NMR (99 MHz, 1:5 DFB:Toluene-dz): $\delta$ 14.4 (s).

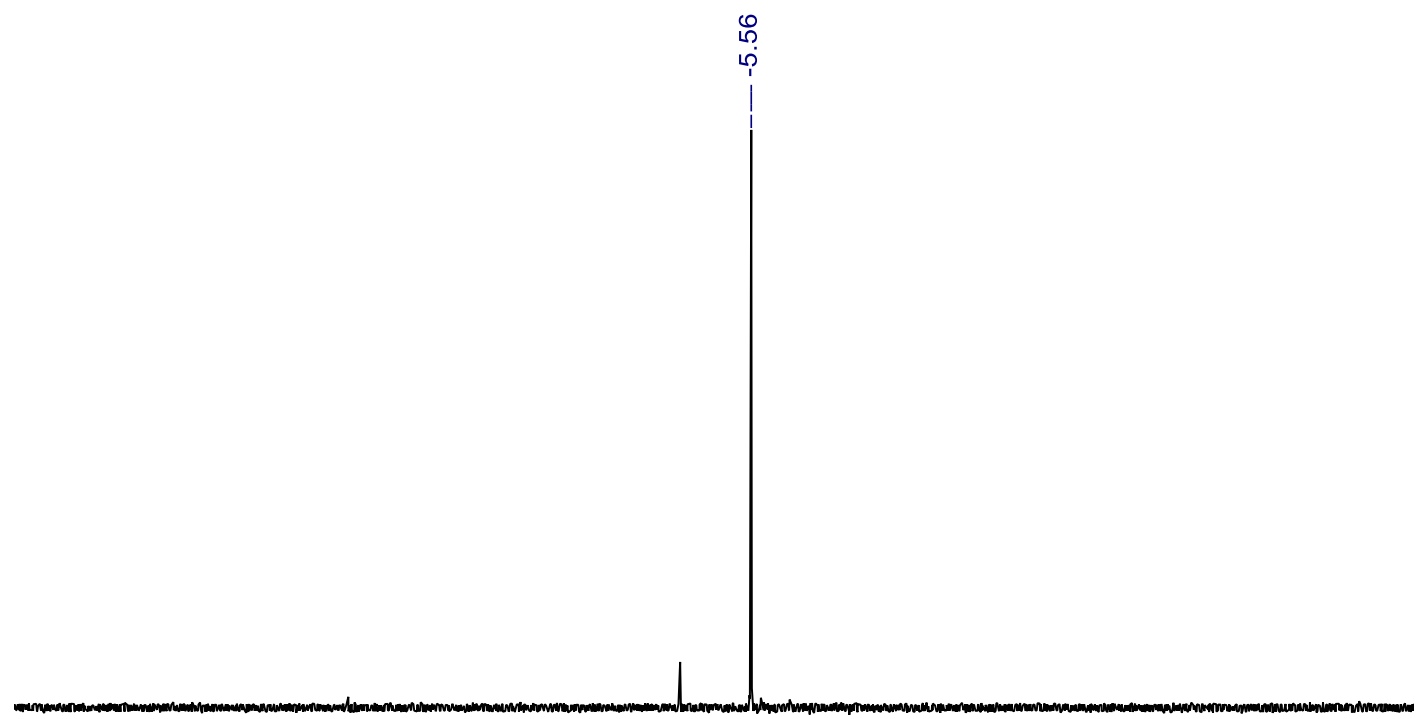

$\begin{array}{llllllllllllllllllll}90 & 80 & 70 & 60 & 50 & 40 & 30 & 20 & 10 & 0 & -10 & -20 & -30 & -40 & -50 & -60 & -70 & -80 & -90 & -1 \\ 31 \mathrm{P}(\mathrm{ppm}) & & & & \end{array}$

Figure S16. ${ }^{31} \mathrm{P}\{\mathrm{H}\}$ NMR spectrum of in situ [EtMe $\left.\mathrm{Si}-\mathrm{P}(p-\mathrm{OMe}-\mathrm{Ph})_{3}\right]^{+}\left[\mathrm{B}\left(\mathrm{C}_{6} \mathrm{~F}_{5}\right)_{4}\right]^{-}$.

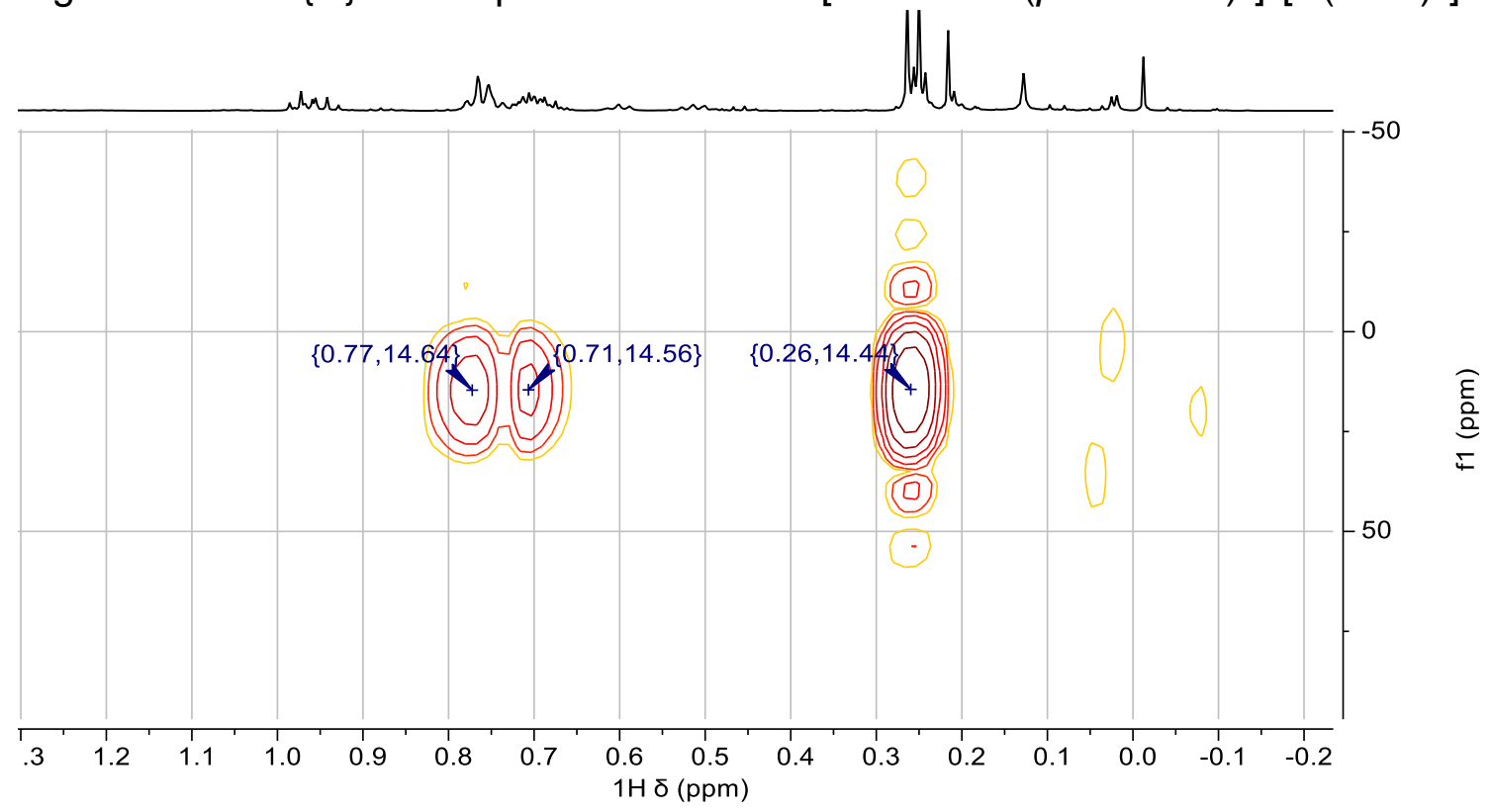

Figure $\mathrm{S} 17 .{ }^{1} \mathrm{H},{ }^{29} \mathrm{Si}-\mathrm{HMBC}$ NMR spectrum of in situ [EtMe $\left.2 \mathrm{Si}-\mathrm{P}(p-\mathrm{OMe}-\mathrm{Ph})_{3}\right]^{+}\left[\mathrm{B}\left(\mathrm{C}_{6} \mathrm{~F}_{5}\right)_{4}\right]^{-}$. 
$\left[\mathrm{EtMe}{ }_{2} \mathrm{Si}-\mathrm{P}\left({ }^{\mathrm{t}} \mathrm{Bu}\right)_{3}\right]^{+}\left[\mathrm{B}\left(\mathrm{C}_{6} \mathrm{~F}_{5}\right)_{4}\right]^{-}(\mathbf{1} \mathrm{h})$

${ }^{1} \mathrm{H}$ NMR $\left(600 \mathrm{MHz}, 1: 5 \mathrm{DFB}: T o l u e n e-d_{8}\right): \delta 0.98\left(\mathrm{~d},{ }^{3} \mathrm{~J}_{H H}=14.1 \mathrm{~Hz}, 27 \mathrm{H},-\mathrm{C}_{4} \mathrm{H}_{9}\right), 0.72$ (m, 3H, Si-CH $\left.\mathrm{CH}_{3}\right), 0.65\left(\mathrm{~m}, 2 \mathrm{H}, \mathrm{Si}-\mathrm{CH}_{2} \mathrm{CH}_{3}\right), 0.17$ (d, $\left.{ }^{3}{ }_{\mathrm{PH}}=5.1 \mathrm{~Hz}, 6 \mathrm{H}, \mathrm{Si}-\mathrm{CH}_{3}\right)$. ${ }^{31} \mathrm{P}\left\{{ }^{1} \mathrm{H}\right\}$ NMR (243 MHz, 1:5 DFB:Toluene- $\left.d 8\right): \delta 30.5$ (s). ${ }^{29} \mathrm{Si}$ NMR $(99 \mathrm{MHz}, 1: 5$ DFB:Toluene- $\left.d_{8}\right): \delta 15.1$ (s).
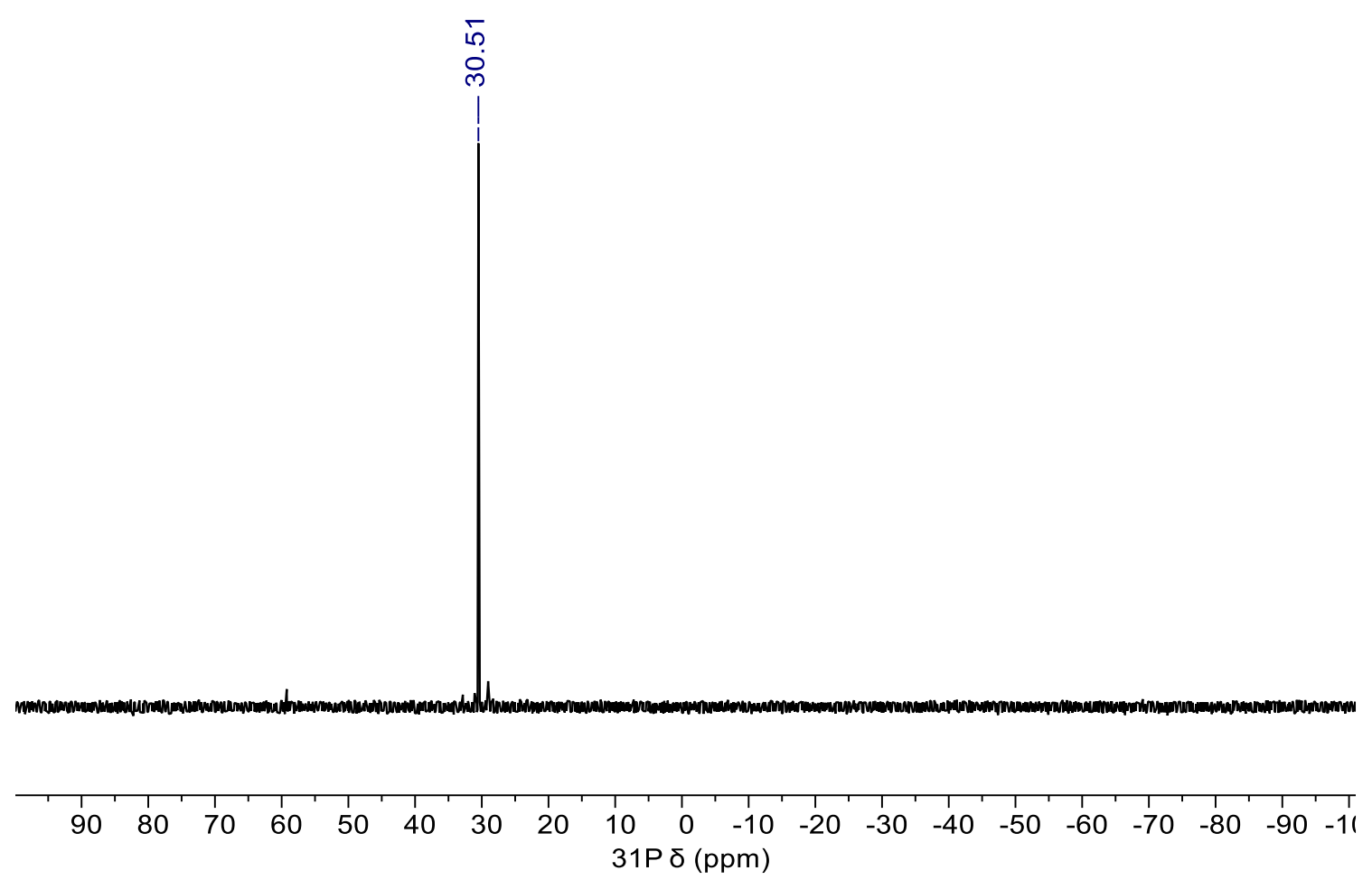

Figure $\mathrm{S} 18 .{ }^{31} \mathrm{P}\{\mathrm{H}\}$ NMR spectrum of in situ $\left[\mathrm{EtMe} 2 \mathrm{Si}-\mathrm{P}\left({ }^{\mathrm{B}} \mathrm{Bu}\right)_{3}\right]^{+}\left[\mathrm{B}\left(\mathrm{C}_{6} \mathrm{~F}_{5}\right)_{4}\right]^{-}$.

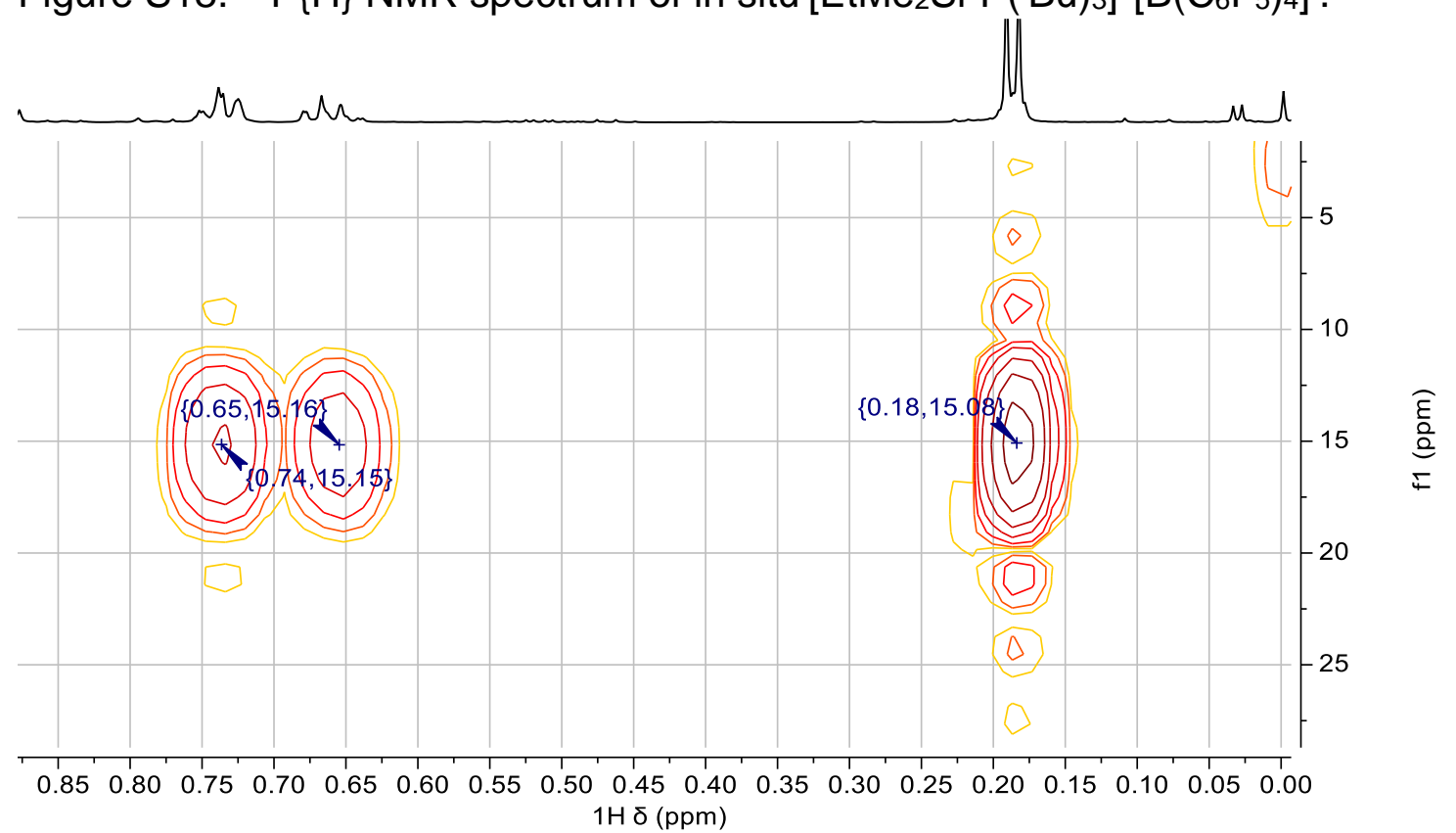

Figure $\mathrm{S} 19 .{ }^{1} \mathrm{H},{ }^{29} \mathrm{Si}-\mathrm{HMBC} \mathrm{HNMR}$ spectrum of in situ $\left[\mathrm{EtMe} 2 \mathrm{Si}-\mathrm{P}\left({ }^{\mathrm{B}} \mathrm{Bu}\right)_{3}\right]^{+}\left[\mathrm{B}\left(\mathrm{C}_{6} \mathrm{~F}_{5}\right)_{4}\right]^{-}$. 
[EtMe2Si-PCy $]^{+}\left[\mathrm{B}\left(\mathrm{C}_{6} \mathrm{~F}_{5}\right)_{4}\right]^{-}(\mathbf{1 i})$

${ }^{1} \mathrm{H}$ NMR (600 MHz, 1:5 DFB:Toluene- $\left.d_{8}\right): \delta 1.90$ (qt, $\left.{ }^{3} J_{H H}=12.7,2.8 \mathrm{~Hz}, 3 \mathrm{H}, \mathrm{Cy}\right), 1.66$ (m, 6H, Cy), 1.56 (m, 9H, Cy), 1.15 (qt, $\left.{ }^{3} \mathrm{~J}_{\mathrm{HH}}=12.2,3.5 \mathrm{~Hz}, 6 \mathrm{H}, \mathrm{Cy}\right), 1.04$ (m, 9H, Cy), 0.79 (td, $\left.{ }^{3} J_{H H}=7.9 \mathrm{~Hz},{ }^{4} J_{P H} 1.6 \mathrm{~Hz}, 3 \mathrm{H}, \mathrm{Si}-\mathrm{CH}_{2} \mathrm{CH}_{3}\right), 0.58\left(\mathrm{qd},{ }^{3} \mathrm{~J}_{\mathrm{HH}}=7.8,{ }^{3} J_{P H} 1.6 \mathrm{~Hz}\right.$, $\left.2 \mathrm{H}, \mathrm{Si}-\mathrm{CH}_{2} \mathrm{CH}_{3}\right), 0.15\left(\mathrm{~d},{ }^{3} J_{P H}=6.1 \mathrm{~Hz}, 6 \mathrm{H}, \mathrm{Si}-\mathrm{CH}_{3}\right) \cdot{ }^{13} \mathrm{C}\{\mathrm{H}\} \mathrm{NMR}(151 \mathrm{MHz}, 1: 5$ DFB:Toluene- $\left.d_{8}\right)$ : $\delta 31.5$ (d, $\left.J=25.3 \mathrm{~Hz}, \mathrm{Cy}\right), 28.6$ (d, $\left.J=3.6 \mathrm{~Hz}, \mathrm{Cy}\right), 26.8(\mathrm{~d}, J=11.2$ $\mathrm{Hz}, \mathrm{Cy}$ ), 25.4 (s, Cy), 7.72 (d, J=7.8 Hz, Si- $\mathrm{CH}_{2} \mathrm{CH}_{3}$ ), 6.16 (d, J=4.9 Hz, Si-CH${ }_{2} \mathrm{CH}_{3}$ ), $2.99\left(\mathrm{~d}, J=7.8 \mathrm{~Hz}, \mathrm{Si}-\mathrm{CH}_{3}\right) \cdot{ }^{31} \mathrm{P}\left\{{ }^{1} \mathrm{H}\right\}$ NMR $\left(243 \mathrm{MHz}, 1: 5 \mathrm{DFB}: T o l u e n e-d_{8}\right): \delta-0.65$ (s). ${ }^{29} \mathrm{Si} \mathrm{NMR}\left(99 \mathrm{MHz}, 1: 5 \mathrm{DFB}: T o l u e n e-d_{8}\right): \delta 10.6$ (s).

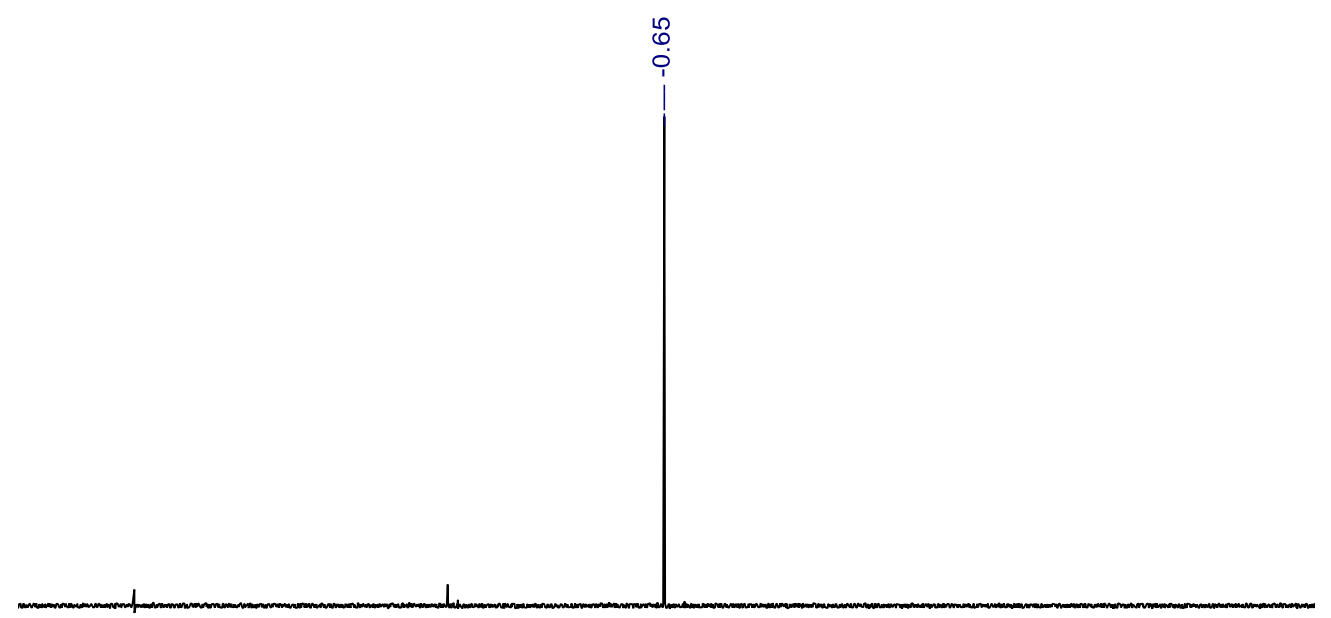

\begin{tabular}{lllllllllllllllllllll}
\hline 00 & 90 & 80 & 70 & 60 & 50 & 40 & 30 & 20 & 10 & 0 & -10 & -20 & -30 & -40 & -50 & -60 & -70 & -80 & -90 & -10 \\
$31 \mathrm{P}(\mathrm{ppm})$ & & &
\end{tabular}

Figure S20. ${ }^{31} \mathrm{P}\{\mathrm{H}\}$ NMR spectrum of in situ $\left[\mathrm{EtMe}_{2} \mathrm{Si}-\mathrm{PC} \mathrm{y}_{3}\right]^{+}\left[\mathrm{B}\left(\mathrm{C}_{6} \mathrm{~F}_{5}\right)_{4}\right]^{-}$.

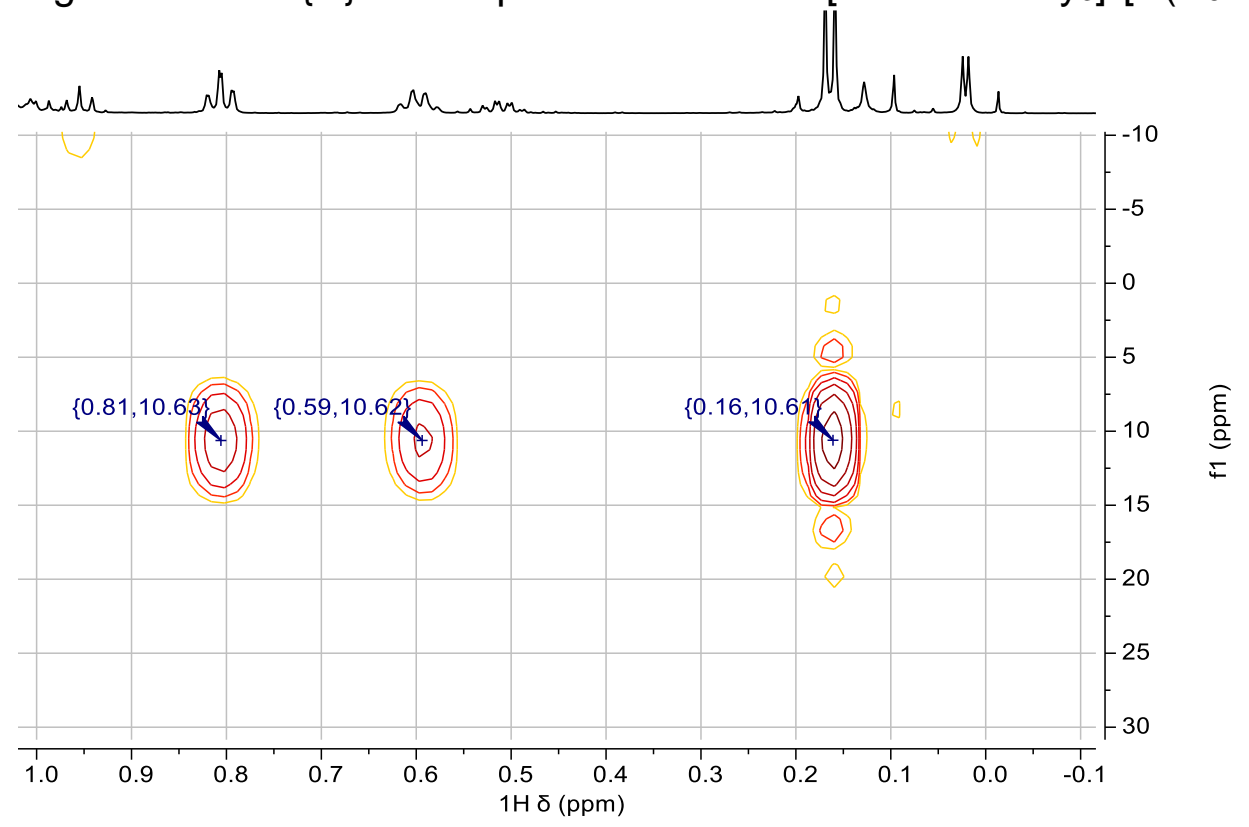

Figure $\mathrm{S} 21 .{ }^{1} \mathrm{H},{ }^{29} \mathrm{Si}-\mathrm{HMBC} \mathrm{HNMR}$ spectrum of in situ $\left[\mathrm{EtMe}_{2} \mathrm{Si}-\mathrm{PC} \mathrm{y}_{3}\right]^{+}\left[\mathrm{B}\left(\mathrm{C}_{6} \mathrm{~F}_{5}\right)_{4}\right]^{-}$. 
$\left[\mathrm{Et}_{2} \mathrm{MeSi}-\mathrm{PPh}_{3}\right]^{+}\left[\mathrm{B}\left(\mathrm{C}_{6} \mathrm{~F}_{5}\right)_{4}\right]^{-}(\mathbf{1 j})$

${ }^{1} \mathrm{H}$ NMR $\left(400 \mathrm{MHz}, 1: 5 \mathrm{DFB}: T o l u e n e-d_{8}\right): \delta 0.64\left(\mathrm{~m}, 10 \mathrm{H}, \mathrm{Si}-\left(\mathrm{CH}_{2} \mathrm{CH}_{3}\right)_{2}\right), 0.15\left(\mathrm{~d},{ }^{3} J_{P H}=\right.$ $\left.8.3 \mathrm{~Hz}, 3 \mathrm{H}, \mathrm{Si}-\mathrm{CH}_{3}\right) .{ }^{31} \mathrm{P}\left\{{ }^{1} \mathrm{H}\right\} \mathrm{NMR}\left(162 \mathrm{MHz}, 1: 5 \mathrm{DFB}: T o l u e n e-d_{8}\right): \delta-3.2$ (s). ${ }^{29} \mathrm{Si} \mathrm{NMR}$ (99 MHz, 1:5 DFB:Toluene-ds): $\delta 17.4$ (s).

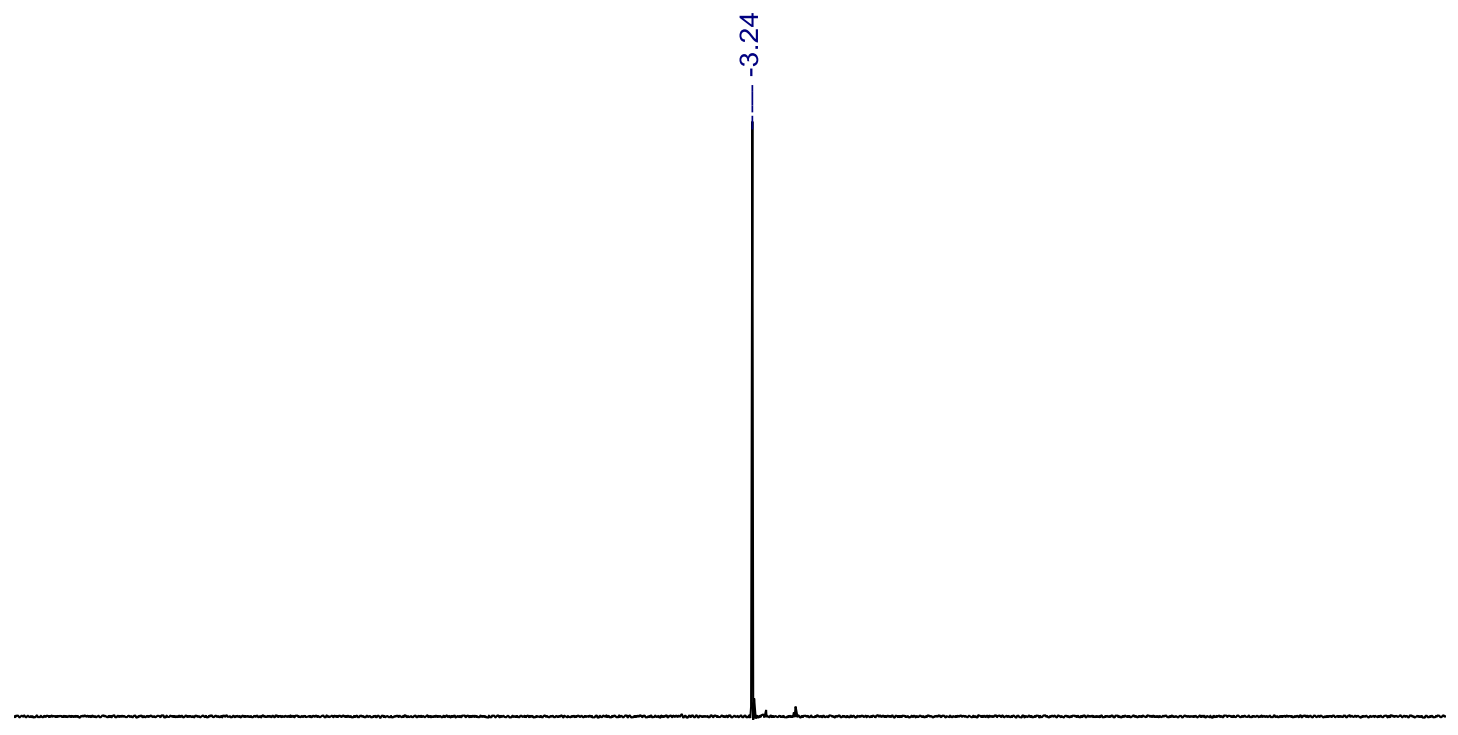

\begin{tabular}{lllllllllllllllllllll}
\hline 00 & 90 & 80 & 70 & 60 & 50 & 40 & 30 & 20 & $\begin{array}{c}10 \\
10\end{array}$ & 0 & -10 & -20 & -30 & -40 & -50 & -60 & -70 & -80 & -90 & -1 \\
$31 \mathrm{P} \delta(\mathrm{ppm})$ & & &
\end{tabular}

Figure S22. ${ }^{31} \mathrm{P}\{\mathrm{H}\}$ NMR spectrum of in situ $\left[\mathrm{Et}_{2} \mathrm{MeSi}-\mathrm{PPh}_{3}\right]^{+}\left[\mathrm{B}\left(\mathrm{C}_{6} \mathrm{~F}_{5}\right)_{4}\right]^{-}$.

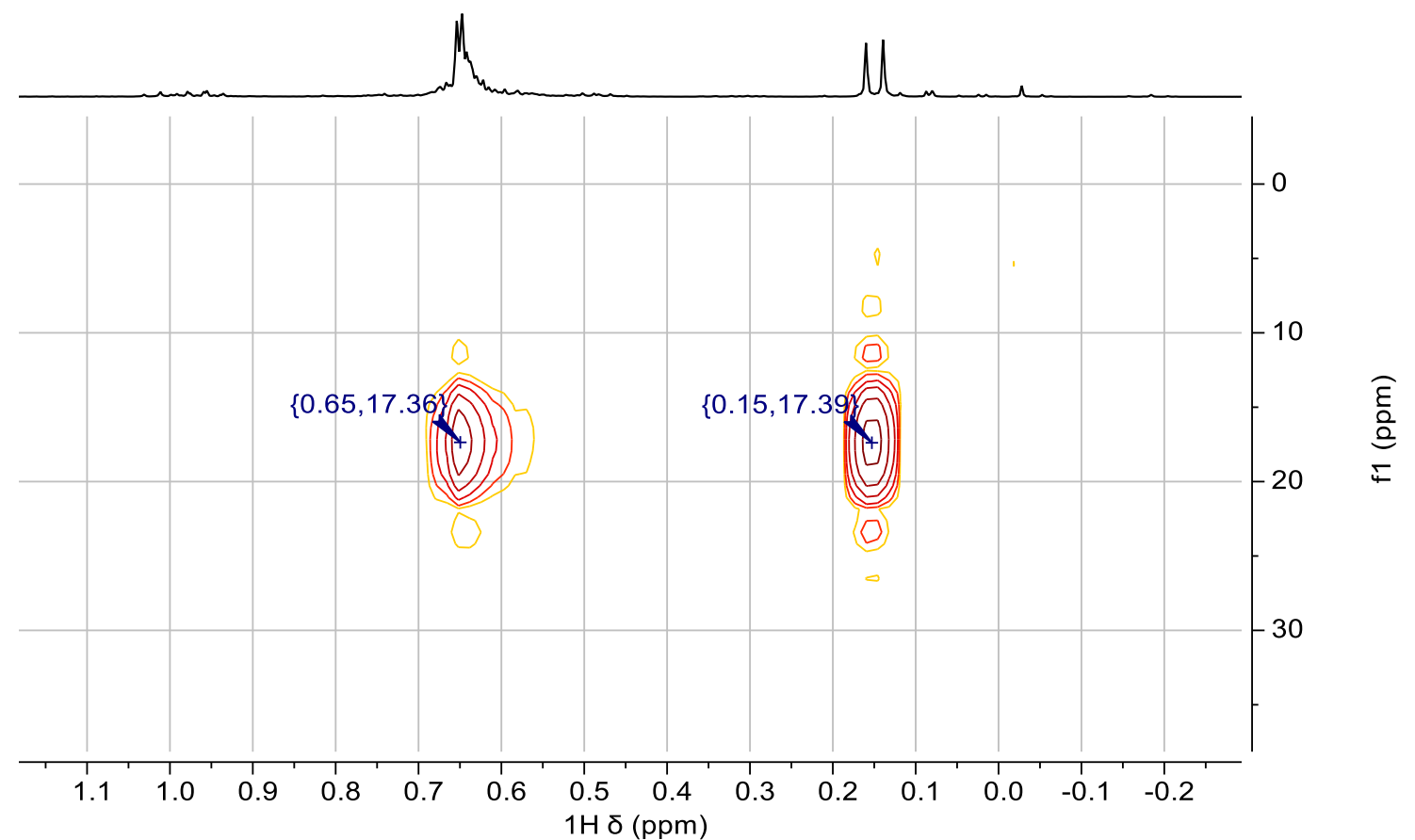

Figure S23. ${ }^{1} \mathrm{H},{ }^{29} \mathrm{Si}-\mathrm{HMBC} \mathrm{HNMR}$ spectrum of in situ $\left[\mathrm{Et}_{2} \mathrm{MeSi}-\mathrm{PPh}_{3}\right]^{+}\left[\mathrm{B}\left(\mathrm{C}_{6} \mathrm{~F}_{5}\right)_{4}\right]^{-}$. 
$\left[\mathrm{Et}_{3} \mathrm{Si}-\mathrm{PPh}\right]^{+}\left[\mathrm{B}\left(\mathrm{C}_{6} \mathrm{~F}_{5}\right)_{4}\right]^{-}(\mathbf{1 k})$

${ }^{1} \mathrm{H}$ NMR $\left(500 \mathrm{MHz}, 1: 5 \mathrm{DFB}: T o l u e n e-d_{8}\right): \delta 0.71\left(\mathrm{~m}, 15 \mathrm{H}, \mathrm{Si}-\left(\mathrm{CH}_{2} \mathrm{CH}_{3}\right)_{3}\right) .{ }^{31} \mathrm{P}\left\{{ }^{1} \mathrm{H}\right\} \mathrm{NMR}$ (202 MHz, 1:5 DFB:Toluene- $d_{8}$ ): $\delta$-3.4 (s). $\left.{ }^{29} \mathrm{Si} \mathrm{NMR} \mathrm{(99} \mathrm{MHz,} \mathrm{1:5} \mathrm{DFB:Toluene-} d_{8}\right): \delta$ 19.0 (s).

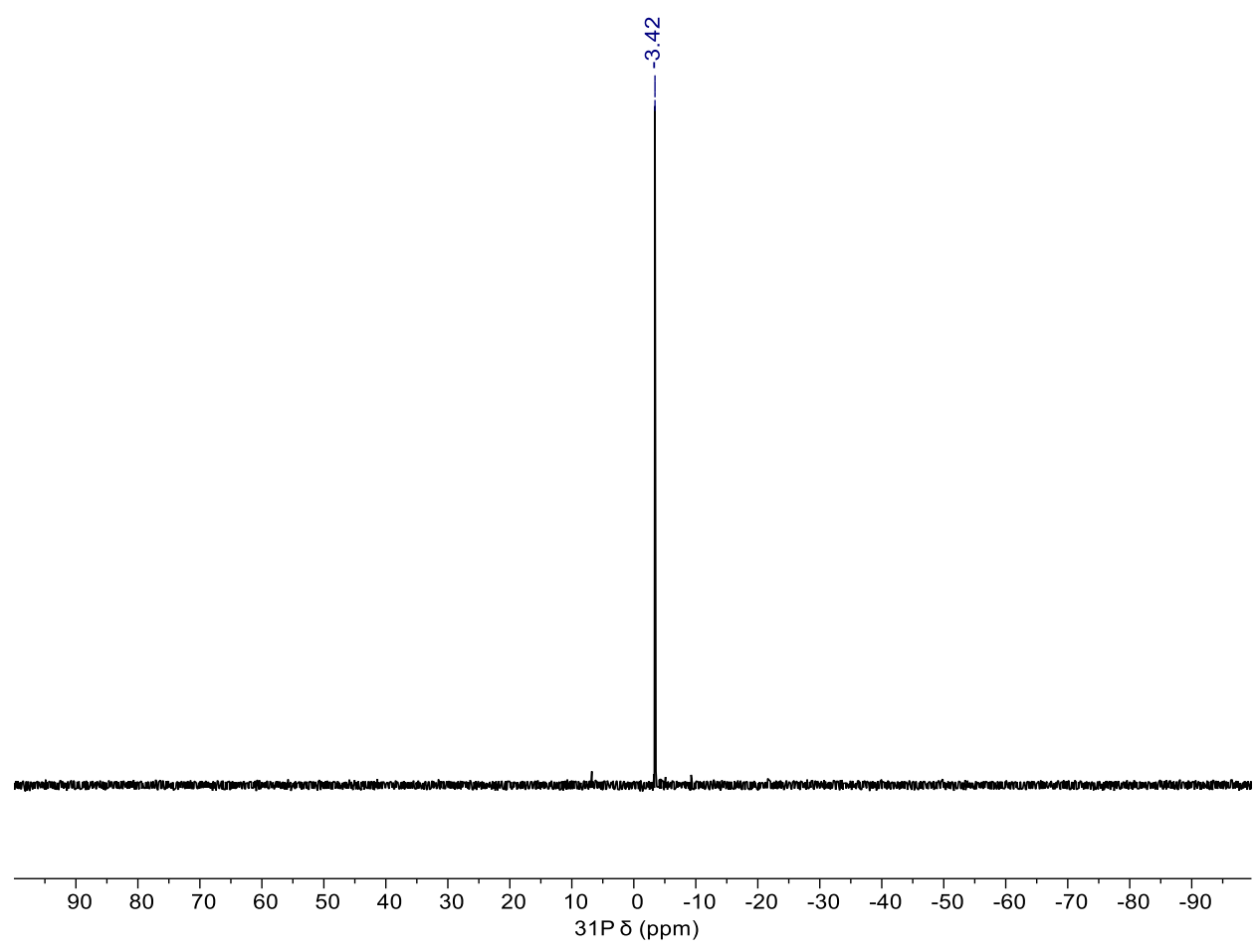

Figure $\mathrm{S} 24 .{ }^{31} \mathrm{P}\{\mathrm{H}\}$ NMR spectrum of in situ $\left[\mathrm{Et}_{3} \mathrm{Si}-\mathrm{PPh}_{3}\right]^{+}\left[\mathrm{B}\left(\mathrm{C}_{6} \mathrm{~F}_{5}\right)_{4}\right]^{\text {. }}$.

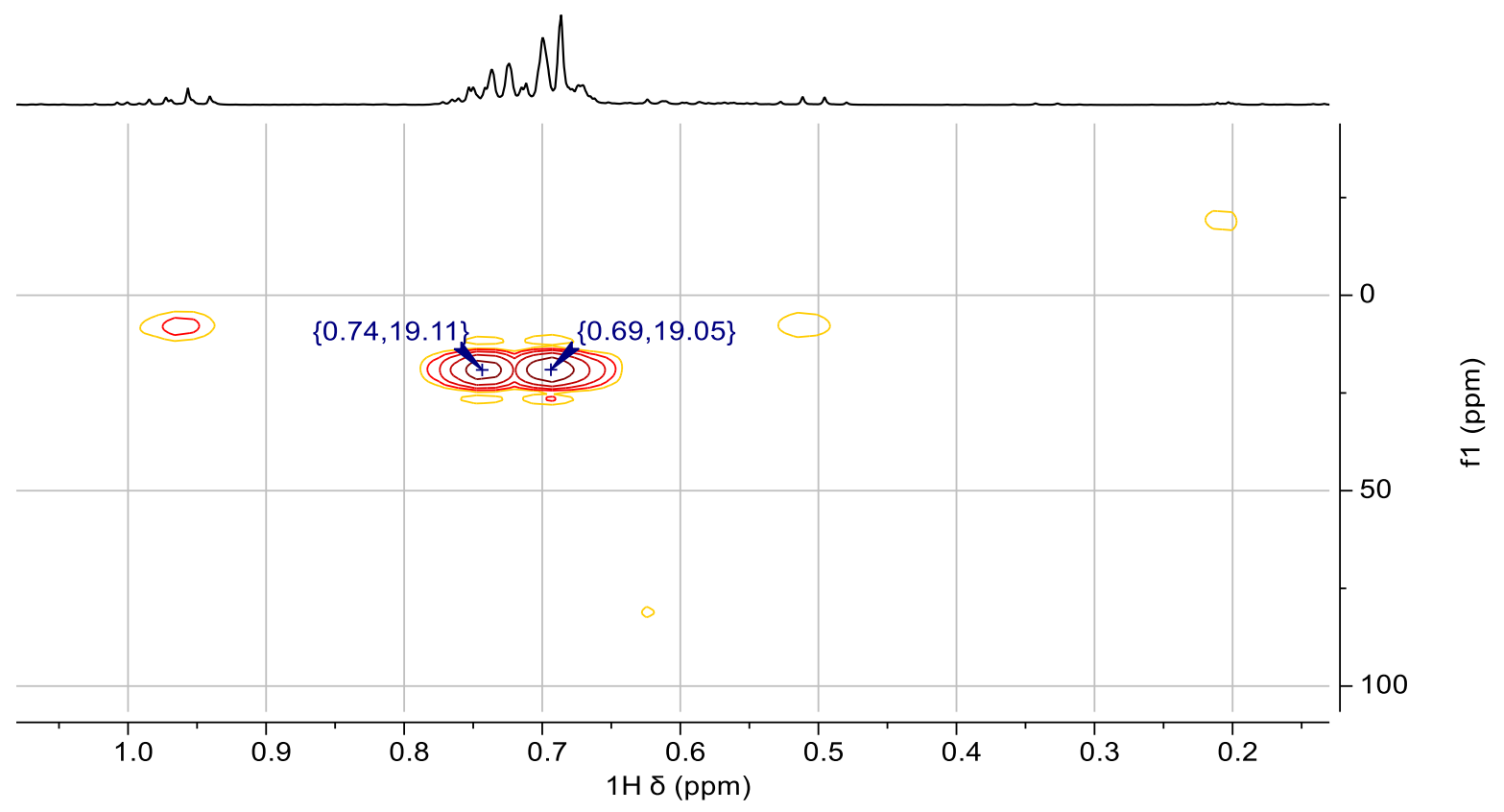

Figure $\mathrm{S} 25 .{ }^{1} \mathrm{H},{ }^{29} \mathrm{Si}-\mathrm{HMBC} \mathrm{HNMR}$ spectrum of in situ $\left[\mathrm{Et}{ }_{3} \mathrm{Si}-\mathrm{PPh}_{3}\right]^{+}\left[\mathrm{B}\left(\mathrm{C}_{6} \mathrm{~F}_{5}\right) 4\right]^{-}$. 


\section{$\left.{ }^{\mathrm{t} B u M e}{ }_{2} \mathrm{Si}-\mathrm{PPh}_{3}\right]^{+}\left[\mathrm{B}\left(\mathrm{C}_{6} \mathrm{~F}_{5}\right)_{4}\right]^{-}(1 \mathrm{I})$}

${ }^{1} \mathrm{H}$ NMR (400 MHz, 1:5 DFB:Toluene- $\left.d_{8}\right): \delta 0.58\left(\mathrm{~s}, 9 \mathrm{H}, \mathrm{Si}{ }^{\mathrm{t}} \mathrm{Bu}\right), 0.21$ (d, ${ }^{3} \mathrm{~J}_{P H}=8.3 \mathrm{~Hz}$, $\left.6 \mathrm{H}, \mathrm{Si}-\left(\mathrm{CH}_{3}\right)_{2}\right) .{ }^{31} \mathrm{P}\left\{{ }^{1} \mathrm{H}\right\}$ NMR (162 MHz, 1:5 DFB:Toluene- $\left.d_{8}\right): \delta-1.8(\mathrm{~s}) .{ }^{29} \mathrm{Si}$ NMR $(99$ $\mathrm{MHz}, 1: 5$ DFB:Toluene- $\left.d_{8}\right): \delta 20.8$ (s).

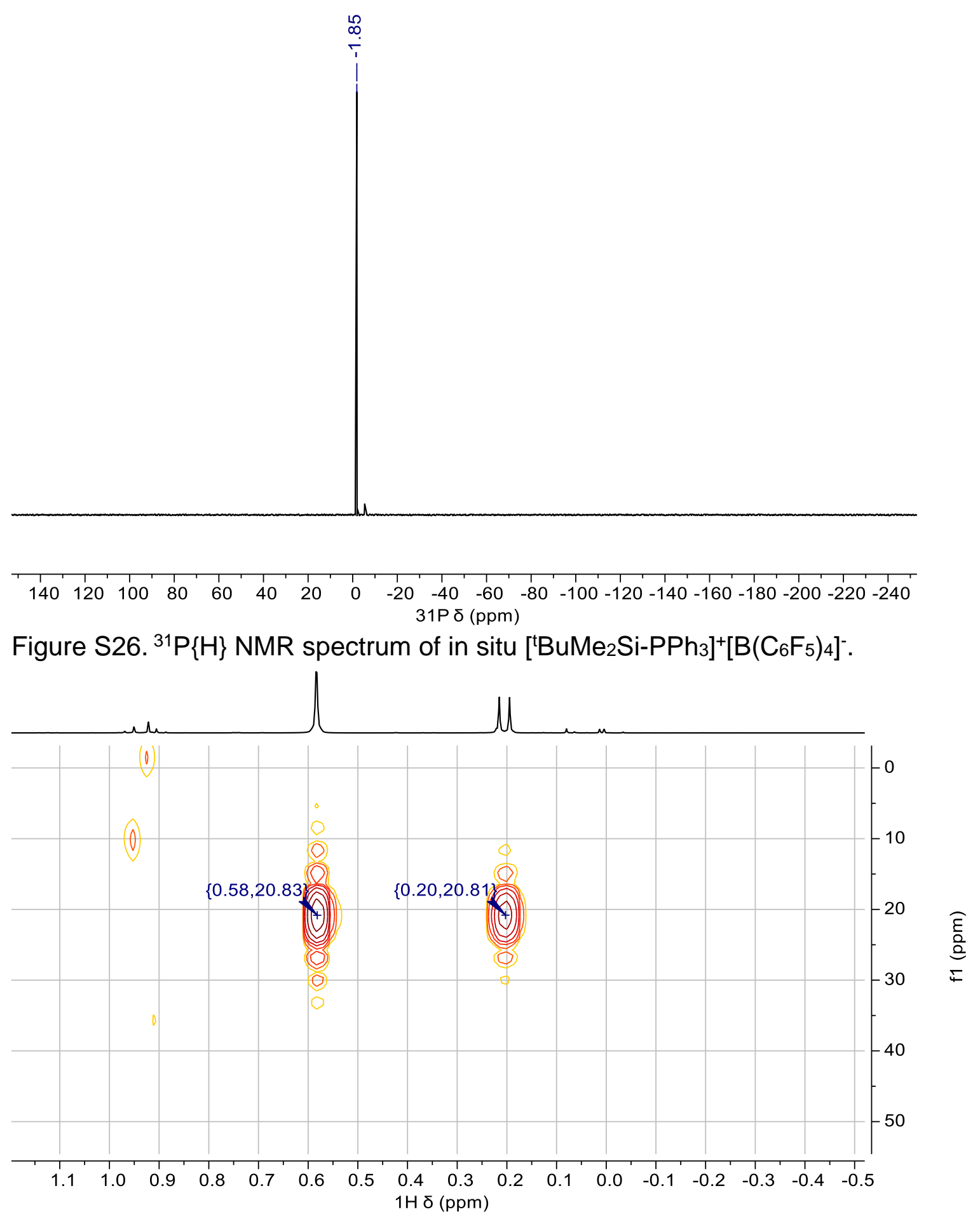

Figure S27. ${ }^{1} \mathrm{H},{ }^{29} \mathrm{Si}-\mathrm{HMBC} \mathrm{HNMR}$ spectrum of in situ [ $\left.{ }^{\mathrm{B} u M} \mathrm{Me}_{2} \mathrm{Si}-\mathrm{PPh}_{3}\right]^{+}\left[\mathrm{B}\left(\mathrm{C}_{6} \mathrm{~F}_{5}\right)_{4}\right]^{-}$. 


\section{$\left.\left[{ }^{i} \mathrm{Pr}_{3} \mathrm{Si}-\mathrm{PPh}\right]^{+}\right]^{+}\left[\mathrm{B}\left(\mathrm{C}_{6} \mathrm{~F}_{5}\right)_{4}\right]^{-}(\mathbf{1 m})$}

${ }^{1} \mathrm{H}$ NMR $\left(400 \mathrm{MHz}, 1: 5 \mathrm{DFB}: T o l u e n e-d_{8}, \delta\right) 1.36$ (qd, ${ }^{3} J_{H H}=7.5,{ }^{3} J_{P H} 4.4,3 \mathrm{H}, \mathrm{Si}-$ $\left.\mathrm{CHC}_{2} \mathrm{H}_{6}\right), 0.82\left(\mathrm{~d},{ }^{3} \mathrm{~J}_{\mathrm{HH}}=7.6 \mathrm{~Hz}, 18 \mathrm{H}, \mathrm{Si}-\mathrm{CHC}_{2} \mathrm{H}_{6}\right) .{ }^{31} \mathrm{P}\left\{{ }^{1} \mathrm{H}\right\} \mathrm{NMR}(162 \mathrm{MHz}, 1: 5$ DFB:Toluene-dz): $\delta$-1.6 (s). ${ }^{29} \mathrm{Si}$ NMR (99 MHz, 1:5 DFB:Toluene- $\left.d_{8}\right): \delta 25.9$ (s).

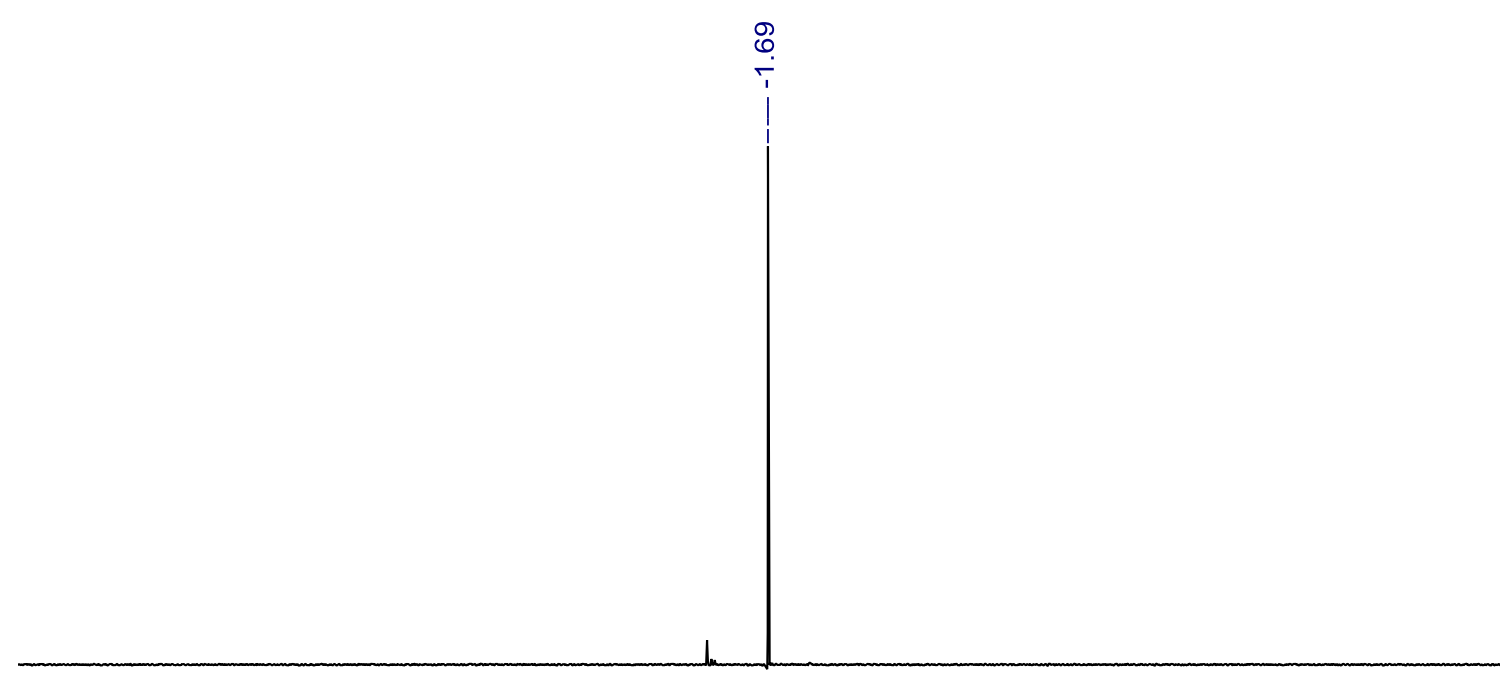

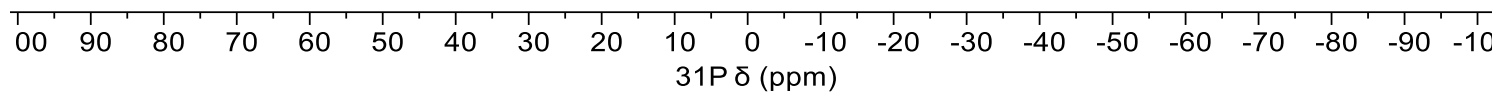

Figure S28. ${ }^{31} \mathrm{P}\{\mathrm{H}\}$ NMR spectrum of in situ [iPr $\left.3 \mathrm{Si}^{-} \mathrm{PPh}_{3}\right]^{+}\left[\mathrm{B}\left(\mathrm{C}_{6} \mathrm{~F}_{5}\right)_{4}\right]^{-}$.

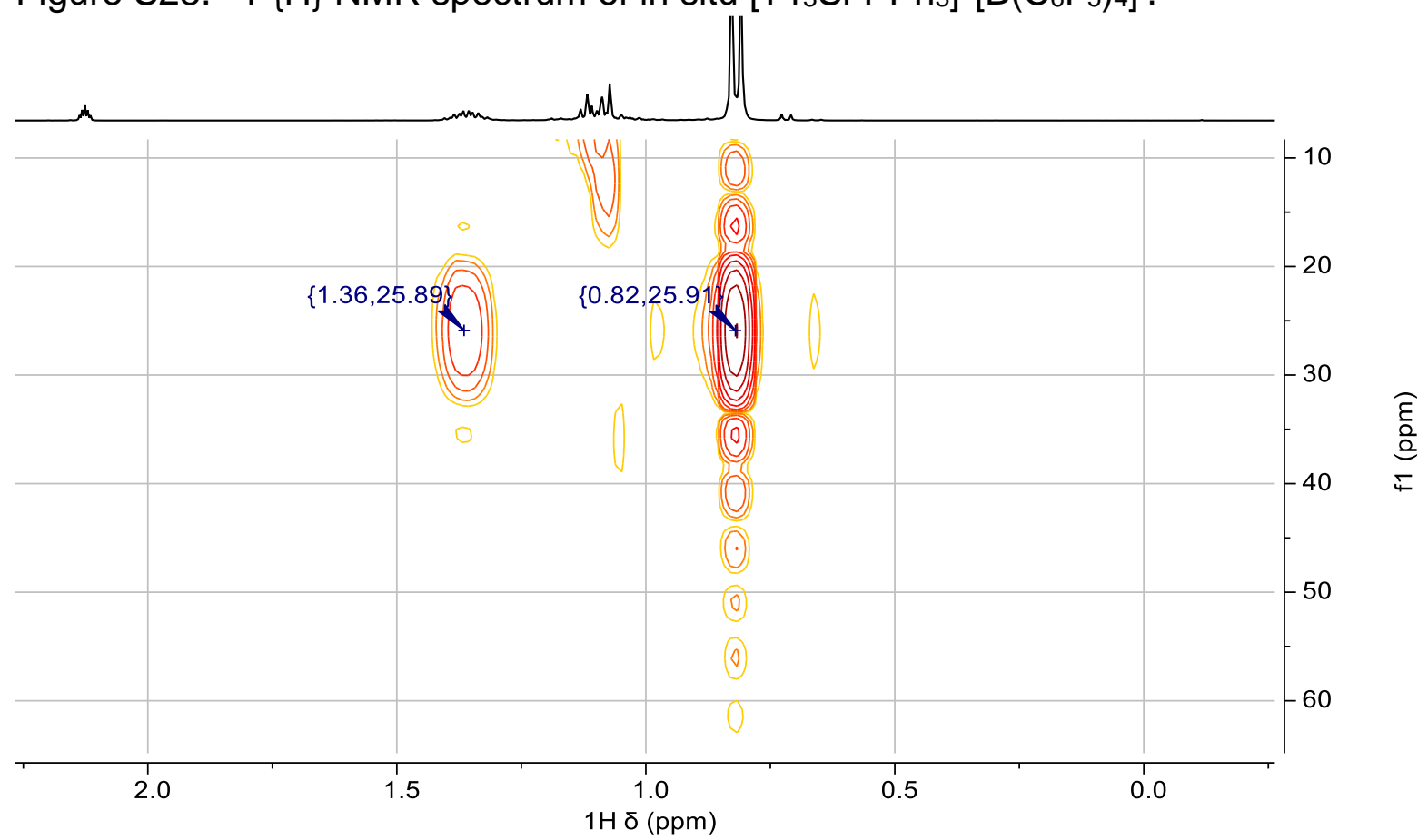

Figure S29. ${ }^{1} \mathrm{H},{ }^{29} \mathrm{Si}-\mathrm{HMBC} \mathrm{HNMR}$ spectrum of in situ $\left[{ }^{i} \mathrm{Pr}_{3} \mathrm{Si}-\mathrm{PPh}_{3}\right]^{+}\left[\mathrm{B}\left(\mathrm{C}_{6} \mathrm{~F}_{5}\right)_{4}\right]^{-}$. 
$\left.{ }^{[}{ }^{\mathrm{Bu}} \mathrm{u}_{3} \mathrm{P}-\mathrm{SiEt}_{3}\right]^{+}\left[\mathrm{B}\left(\mathrm{C}_{6} \mathrm{~F}_{5}\right)_{4}\right]^{-}$(further characterization can be found in the literature) ${ }^{2}$ ${ }^{31} \mathrm{P}\left\{{ }^{1} \mathrm{H}\right\}$ NMR (202 MHz, 1:5 DFB:Toluene- $\left.d 8\right): \delta 36.1(\mathrm{~s}) .{ }^{29} \mathrm{Si} N \mathrm{NR}(99 \mathrm{MHz}, 1: 5$ DFB:Toluene- $\left.d_{8}\right): \delta 21.8(\mathrm{~s})$.

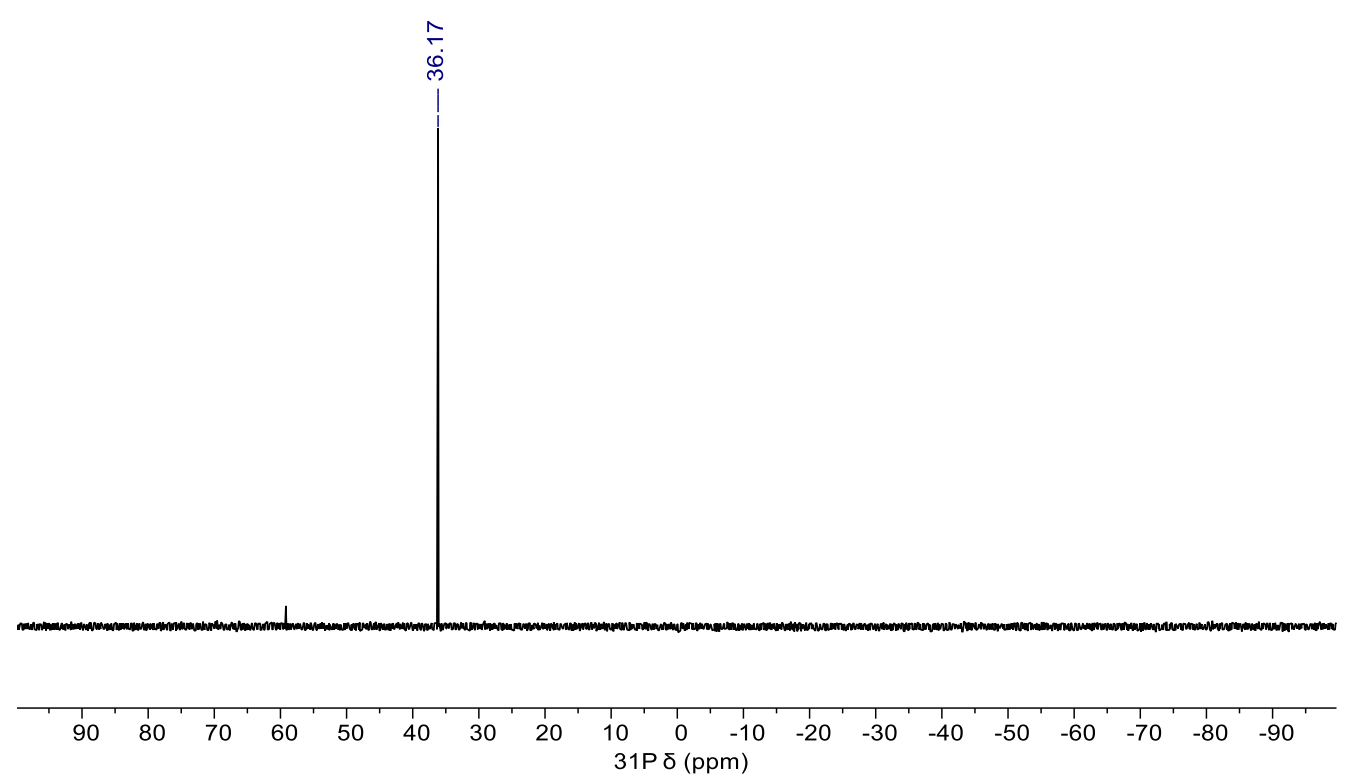

Figure S30. ${ }^{31} \mathrm{P}\{\mathrm{H}\}$ NMR spectrum of in situ $\left.\left[{ }^{\mathrm{t}} \mathrm{Bu} 3 \mathrm{P}-\mathrm{SiEt}\right]_{3}\right]^{+}\left[\mathrm{B}\left(\mathrm{C}_{6} \mathrm{~F}_{5}\right)_{4}\right]^{-}$.

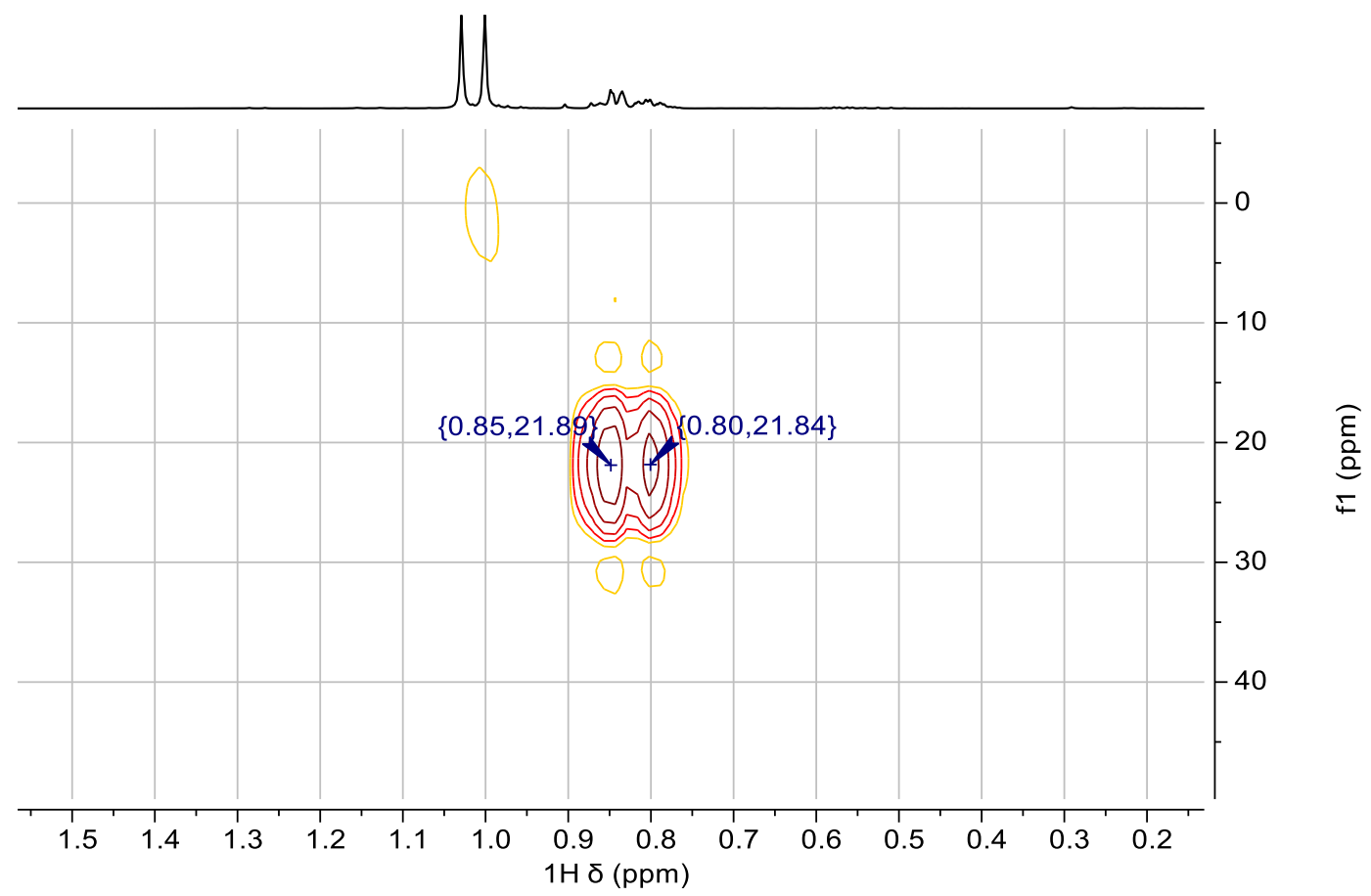

Figure $\mathrm{S} 31 .{ }^{1} \mathrm{H},{ }^{29} \mathrm{Si}-\mathrm{HMBC} \mathrm{HNMR}$ spectrum of in situ $\left[{ }^{\mathrm{t}} \mathrm{Bu} 33 \mathrm{P}-\mathrm{SiEt}\right]^{+}\left[\mathrm{B}\left(\mathrm{C}_{6} \mathrm{~F}_{5}\right)_{4}\right]^{-}$. 


\section{TRANSFER REACTIONS}

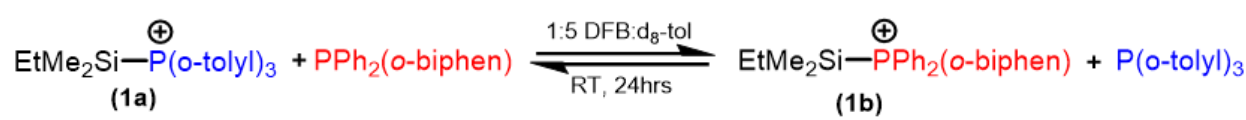
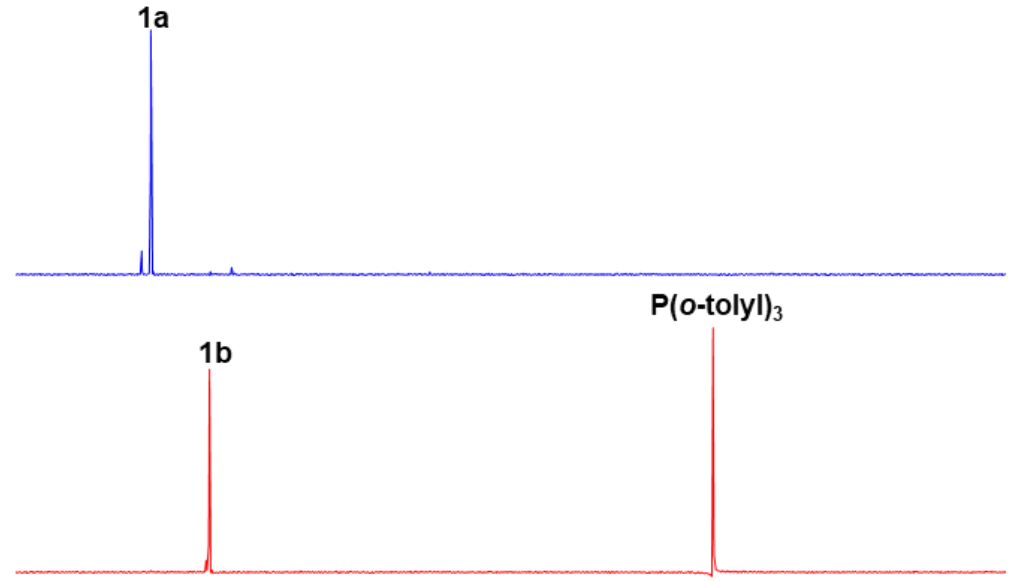

\begin{tabular}{lllllllllllllll}
\hline 10 & 5 & 0 & -5 & -10 & -15 & -20 & -25 & -30 & -35 & -40 & -45
\end{tabular}

Figure S32. ${ }^{31} \mathrm{P}\left\{{ }^{1} \mathrm{H}\right\}$ NMR spectra detailing the stoichiometric transfer of $\mathrm{Me}_{2} \mathrm{EtSi}^{+}$from $\left[\mathrm{Me}_{2} \mathrm{EtSi}-\right.$ $\left.\mathrm{P}(\text { o-tolyl })_{3}\right]^{+}\left(2.52 \mathrm{ppm}\right.$, blue spectra) to $\left[\mathrm{Me}_{2} \mathrm{EtSi}^{-} \mathrm{PPh}_{2} \text { (o-biphen) }\right]^{+}(-0.86 \mathrm{ppm}$, red spectra). At equilibrium no visible $\left[\mathrm{Me}_{2} \mathrm{EtSi}-\mathrm{P}(\mathrm{o} \text {-tolyl })_{3}\right]^{+}$is observed indicating a stoichiometric transfer. Assessment of detection limits indicate that the equilibrium for transfer is $>20$.
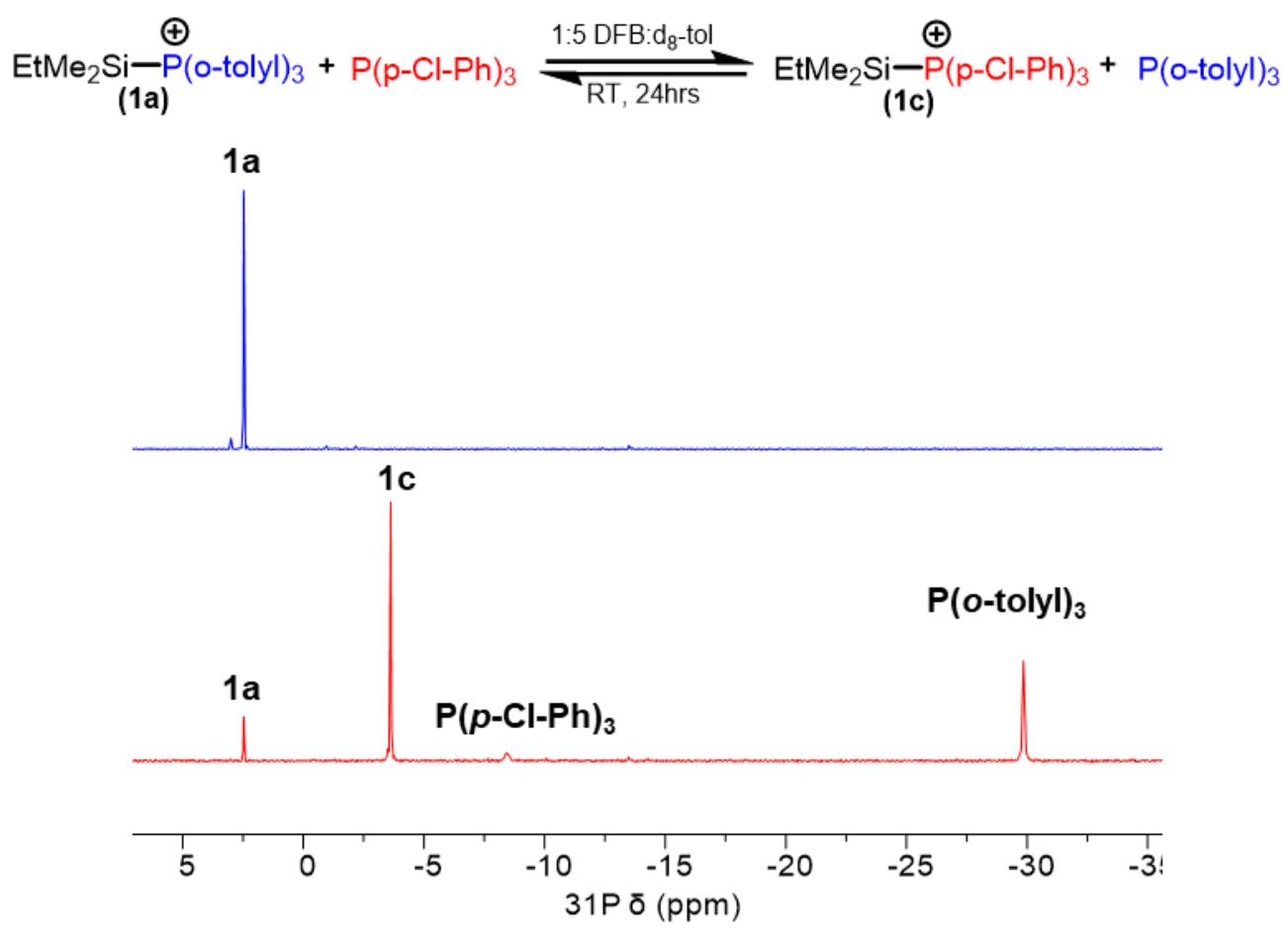

Figure S33. ${ }^{31} \mathrm{P}\left\{{ }^{1} \mathrm{H}\right\}$ NMR spectra detailing the stoichiometric transfer of $\mathrm{Me}_{2} \mathrm{EtSi}^{+}$from $\left[\mathrm{Me}_{2} \mathrm{EtSi}-\mathrm{P}(\text { o-tolyl })_{3}\right]^{+}\left(2.52 \mathrm{ppm}\right.$, blue spectra) to $\left[\mathrm{Me}_{2} \mathrm{EtSi}-\mathrm{P}(\mathrm{p}-\mathrm{Cl}-\mathrm{Ph})_{3}\right]^{+}(-3.61 \mathrm{ppm}$, red spectra). At equilibrium [Me $\left.\mathrm{MetSi}_{2} \mathrm{P}(0-\text { tolyl })_{3}\right]^{+}$is observed indicating a partial transfer. Assessment of detection limits indicate that the equilibrium for transfer is 9.0. 
$\oplus$ $\mathrm{EtMe}_{2} \mathrm{Si}-\mathrm{PPh}_{2}$ (o-biphen) $+\mathrm{P}(\mathrm{p}-\mathrm{Cl}-\mathrm{Ph})_{3}$

(1b)
1:5 DFB: $\mathrm{d}_{8}$-tol

RT, 24hrs

$\oplus$

$\mathrm{EtMe}_{2} \mathrm{Si}-\mathrm{P}(\mathrm{p}-\mathrm{Cl}-\mathrm{Ph})_{3}+\mathrm{PPh}_{2}$ (o-biphen)

(1c)

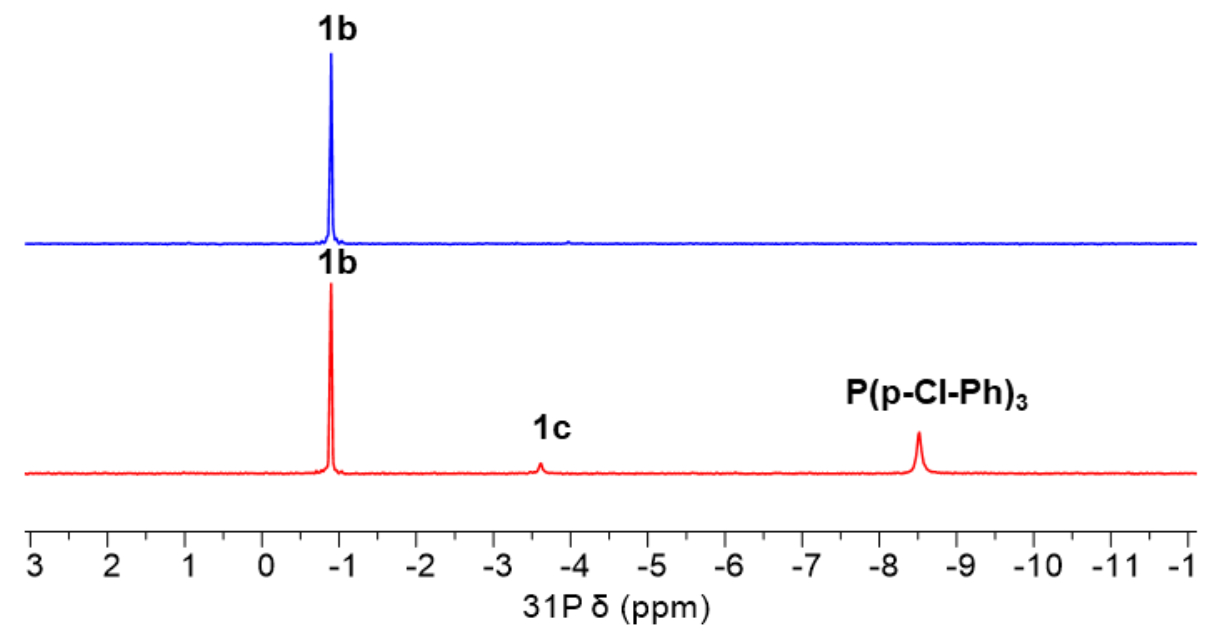

Figure S34. ${ }^{31} \mathrm{P}\left\{{ }^{1} \mathrm{H}\right\}$ NMR spectra detailing the stoichiometric transfer of $\mathrm{Me}_{2} \mathrm{EtSi}^{+}$from $\left[\mathrm{Me}_{2} \mathrm{EtSi}-\right.$ $\mathrm{PPh}_{2}$ (o-biphen) $]^{+}\left(-0.87 \mathrm{ppm}\right.$, blue spectra) to $\left[\mathrm{Me}_{2} \mathrm{EtSi}-\mathrm{P}(\mathrm{p}-\mathrm{Cl}-\mathrm{Ph})_{3}\right]^{+}(-3.61 \mathrm{ppm}$, red spectra). At equilibrium $\left[\mathrm{Me}_{2} \mathrm{EtSi}-\mathrm{PPh}_{2}\right.$ (o-biphen)] ${ }^{+}$is observed indicating a partial transfer. Assessment of detection limits indicate that the equilibrium for transfer is 0.15 .

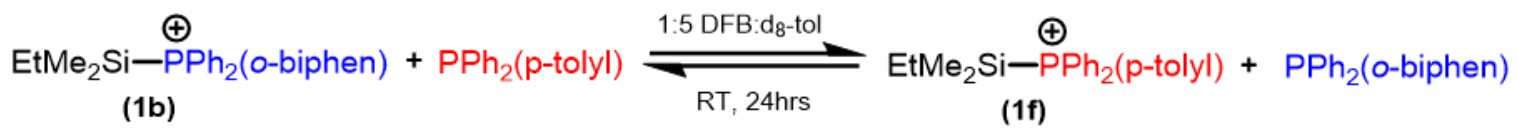

(1b)

1b

\section{(1f)}

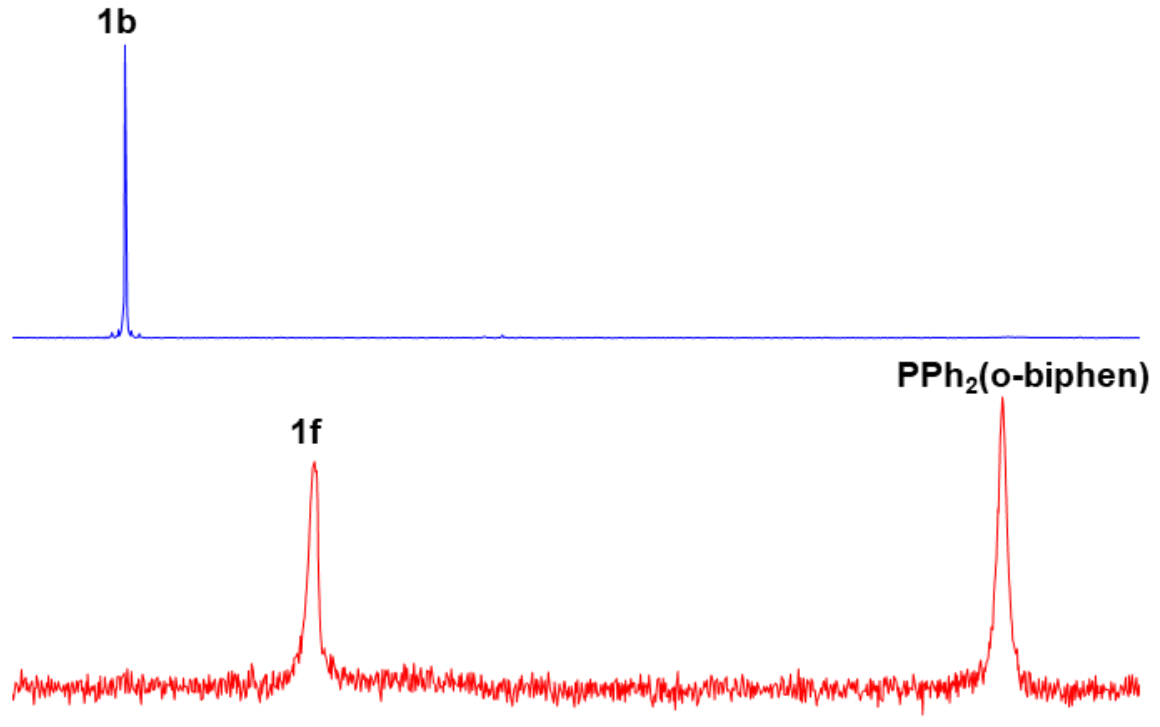

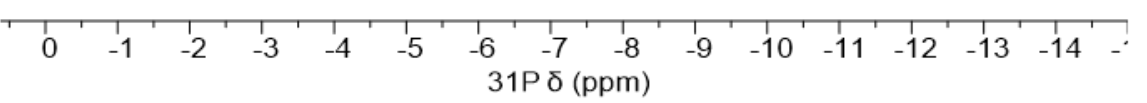

Figure S35. ${ }^{31} \mathrm{P}\left\{{ }^{1} \mathrm{H}\right\}$ NMR spectra detailing the stoichiometric transfer of $\mathrm{Me}_{2} \mathrm{EtSi}^{+}$from $\left[\mathrm{Me}_{2} \mathrm{EtSi}-\mathrm{P}(\mathrm{o}-\right.$ biphen) $\left.\mathrm{Ph}_{2}\right]^{+}\left(-0.86 \mathrm{ppm}\right.$, blue spectra) to $\left[\mathrm{Me}_{2} \mathrm{EtSi}-\mathrm{P}(\mathrm{p} \text {-tolyl }) \mathrm{Ph}_{2}\right]^{+}(-3.53 \mathrm{ppm}$, red spectra). At equilibrium no visible $\left[\mathrm{Me}_{2} \mathrm{EtSi}-\mathrm{P}(\mathrm{o}-\text { biphen }) \mathrm{Ph}_{2}\right]^{+}$is observed indicating stochiometric transfer. Assessment of detection limits indicate that the equilibrium for transfer is $>20$. 


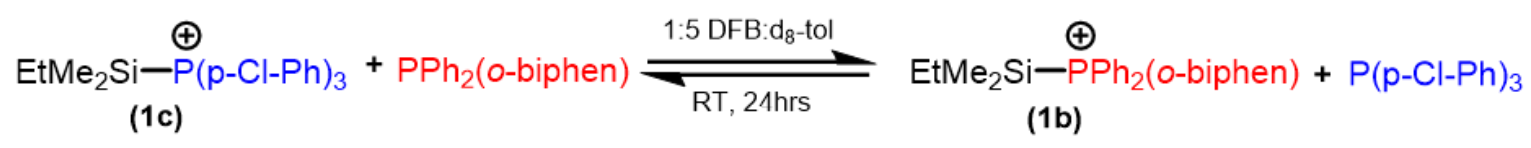

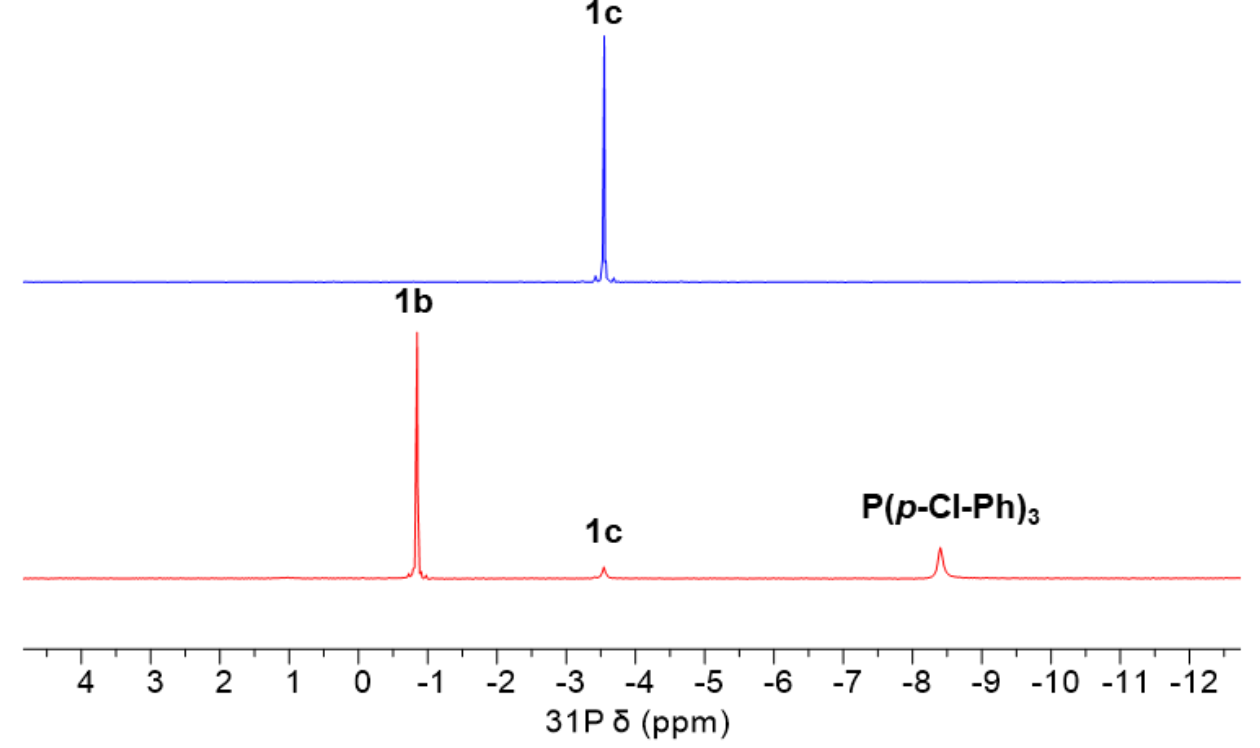

Figure S36. ${ }^{31} \mathrm{P}\left\{{ }^{1} \mathrm{H}\right\}$ NMR spectra detailing the stoichiometric transfer of $\mathrm{Me}_{2} \mathrm{EtSi}^{+}$from $\left[\mathrm{Me}_{2} \mathrm{EtSi}-\mathrm{P}(\mathrm{p}-\mathrm{Cl}-\mathrm{Ph})_{3}\right]^{+}\left(-3.61 \mathrm{ppm}\right.$, blue spectra) to $\left[\mathrm{Me}_{2} \mathrm{EtSi}^{-}-\mathrm{PPh}_{2} \text { (o-biphen) }\right]^{+}(-0.87 \mathrm{ppm}$, red spectra). At equilibrium $\left[\mathrm{Me}_{2} \mathrm{EtSi}-\mathrm{P}(p-\mathrm{Cl}-\mathrm{Ph})_{3}\right]^{+}$is observed indicating partial transfer. Assessment of detection limits indicate that the equilibrium for transfer is 6.6 .

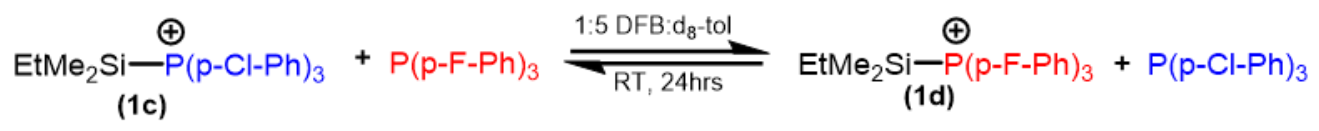
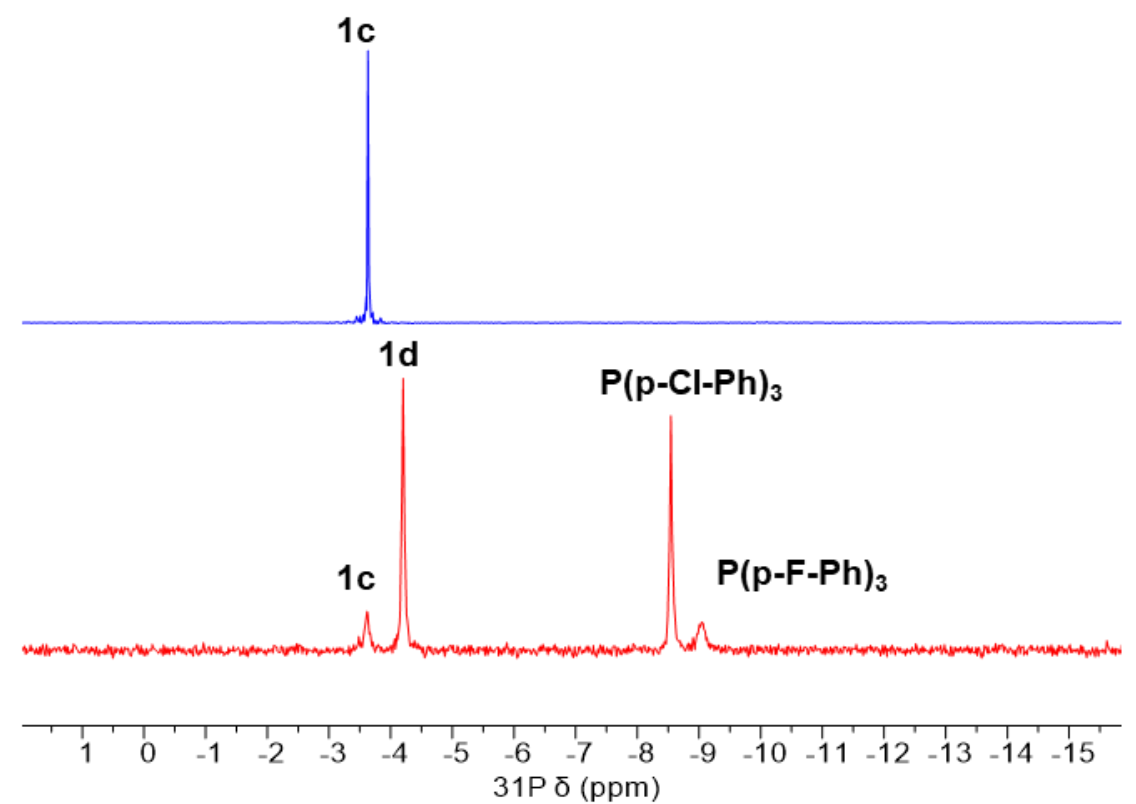

Figure S37. ${ }^{31} \mathrm{P}\left\{{ }^{1} \mathrm{H}\right\}$ NMR spectra detailing the stoichiometric transfer of $\mathrm{Me}_{2} \mathrm{EtSi}^{+}$from $\left[\mathrm{Me}_{2} \mathrm{EtSi}-\mathrm{P}(p-\mathrm{Cl}-\mathrm{Ph})_{3}\right]^{+}\left(-3.61 \mathrm{ppm}\right.$, blue spectra) to $\left[\mathrm{Me}_{2} \mathrm{EtSi}-\mathrm{P}(p-\mathrm{F}-\mathrm{Ph})_{3}\right]^{+}(-4.20 \mathrm{ppm}$, red spectra). At equilibrium $\left[\mathrm{Me}_{2} \mathrm{EtSi}-\mathrm{P}(p-\mathrm{Cl}-\mathrm{Ph})_{3}\right]^{+}$is observed indicating a partial transfer. Assessment of detection limits indicate that the equilibrium for transfer is 3.4 . 


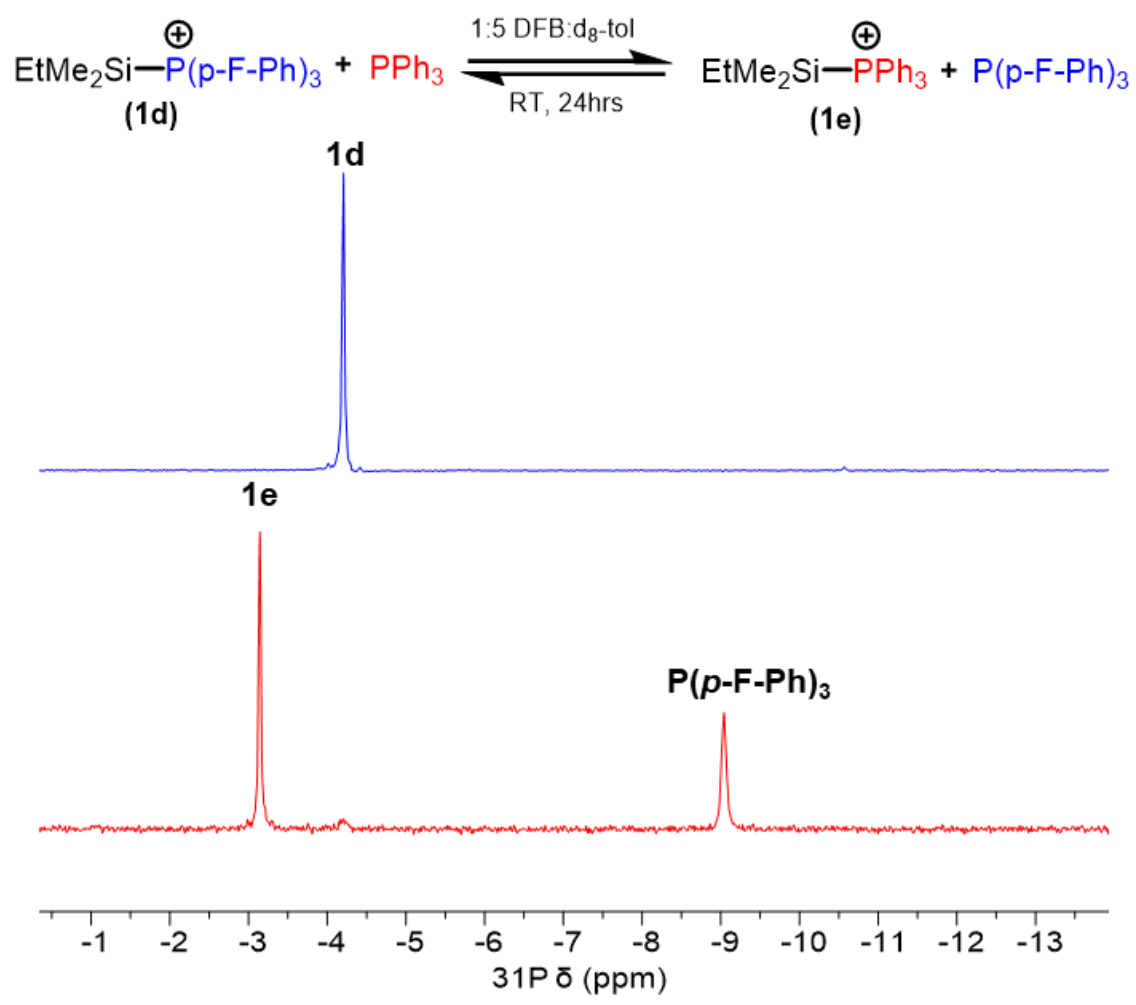

Figure S38. ${ }^{31} \mathrm{P}\left\{{ }^{1} \mathrm{H}\right\}$ NMR spectra detailing the stoichiometric transfer of $\mathrm{Me}_{2} \mathrm{EtSi}^{+}$from $\left[\mathrm{Me}_{2} \mathrm{EtSi}-\mathrm{P}(\mathrm{p}-\mathrm{F}-\mathrm{Ph})_{3}\right]^{+}\left(-4.20 \mathrm{ppm}\right.$, blue spectra) to $\left[\mathrm{Me}_{2} \mathrm{EtSi}-\mathrm{PPh}_{3}\right]^{+}(-3.14 \mathrm{ppm}$, red spectra). At equilibrium $\left[\mathrm{Me}_{2} \mathrm{EtSi}-\mathrm{P}(\mathrm{p}-\mathrm{F}-\mathrm{Ph})_{3}\right]^{+}$is observed indicating a partial transfer. Assessment of detection limits indicate that the equilibrium for transfer is 10 .

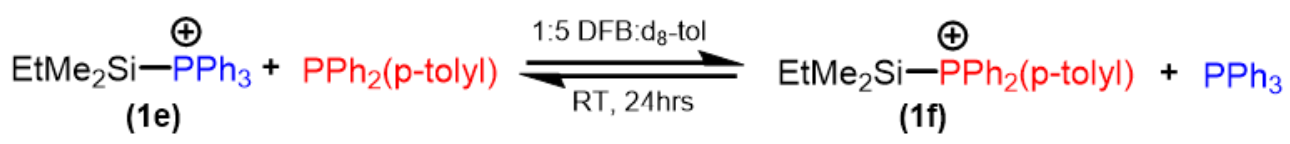

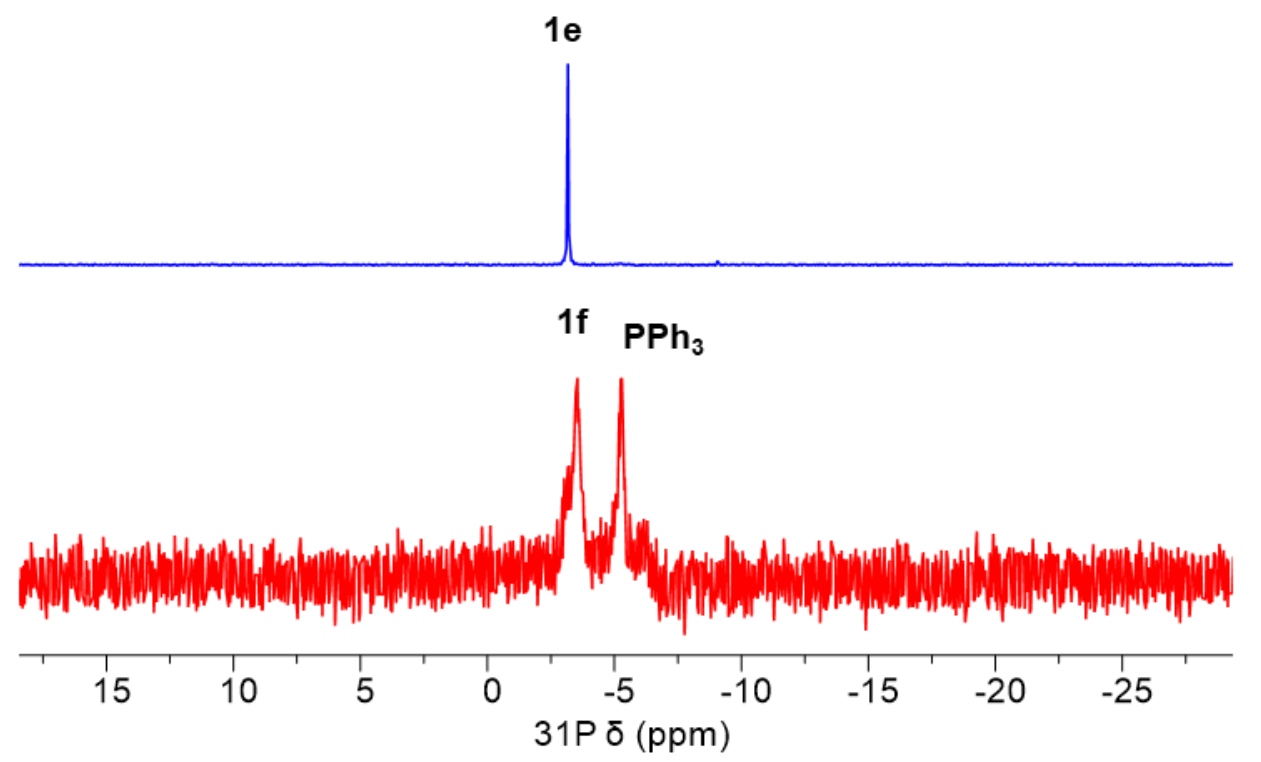

Figure S39. ${ }^{31} \mathrm{P}\left\{{ }^{1} \mathrm{H}\right\}$ NMR spectra detailing the stoichiometric transfer of $\mathrm{Me}_{2} \mathrm{EtSi}^{+}$from $\left[\mathrm{Me}_{2} \mathrm{EtSi}_{-\mathrm{PPh}}\right]^{+}\left(-3.14 \mathrm{ppm}\right.$, blue spectra) to $\left[\mathrm{Me}_{2} \mathrm{EtSi}_{-} \mathrm{PPh}_{2}\right.$ (p-tolyl) ${ }^{+}(-3.53 \mathrm{ppm}$, red spectra). At equilibrium no visible $\left[\mathrm{Me}_{2} \mathrm{EtSi}-\mathrm{PPh}_{3}\right]^{+}$is observed indicating a stoichiometric transfer. Assessment of detection limits indicate that the equilibrium for transfer is $>20$. 


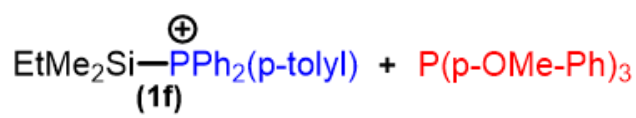
1 $\oplus$
1:5 DFB: $\mathrm{d}_{8}$-tol

RT, 24hrs
$\mathrm{EtMe}_{2} \mathrm{Si}-\mathrm{P}($

(1g)

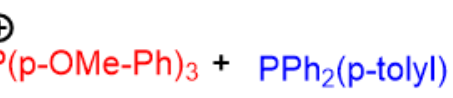

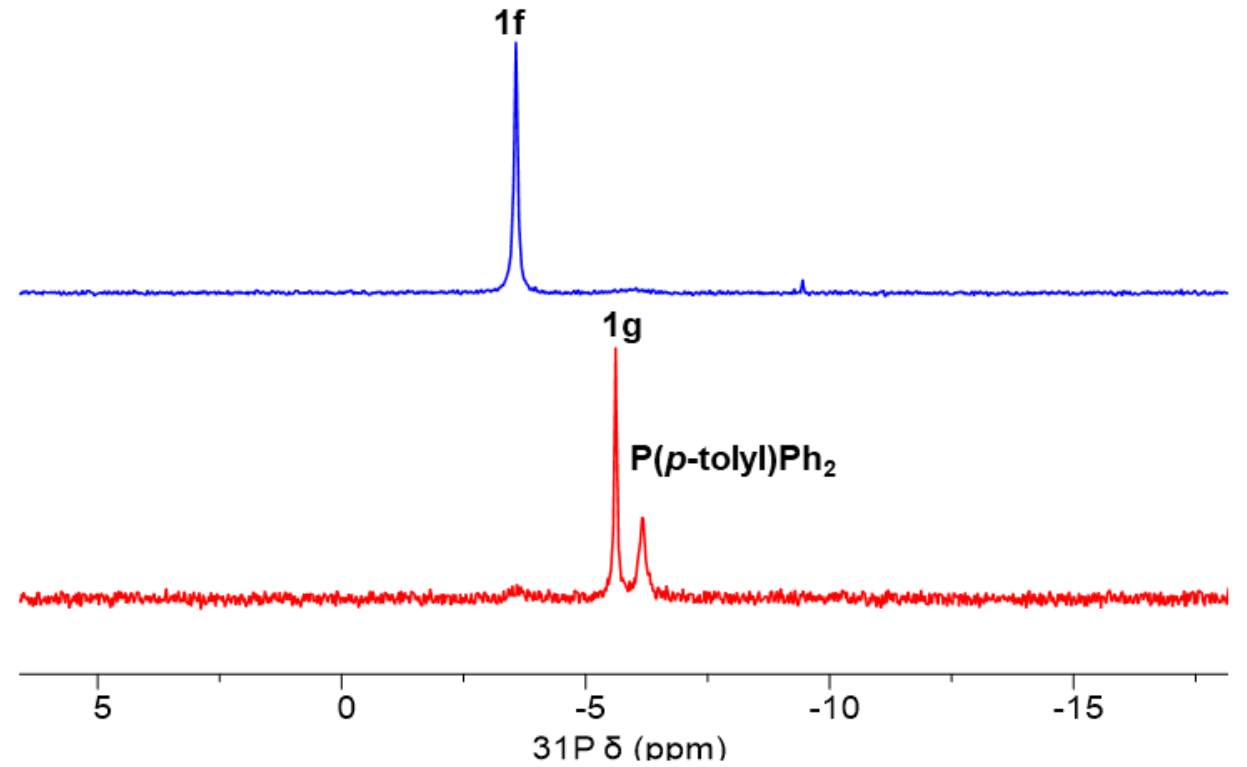

Figure S40. ${ }^{31} \mathrm{P}\left\{{ }^{1} \mathrm{H}\right\}$ NMR spectra detailing the stoichiometric transfer of $\mathrm{Me}_{2} \mathrm{EtSi}^{+}$from $\left[\mathrm{Me}_{2} \mathrm{EtSi}-\right.$ $\mathrm{PPh}_{2}(p$-tolyl $\left.)\right]^{+}\left(-3.53 \mathrm{ppm}\right.$, blue spectra) to $\left[\mathrm{Me}_{2} \mathrm{EtSi}-\mathrm{P}(p-\mathrm{OMe}-\mathrm{Ph})_{3}\right]^{+}(-5.61 \mathrm{ppm}$, red spectra). At equilibrium no visible $\left[\mathrm{Me}_{2} \mathrm{EtSi}_{\mathrm{SPPh}}(p \text {-tolyl) }]^{+}\right.$is observed indicating a stoichiometric transfer. Assessment of detection limits indicate that the equilibrium for transfer is $>20$.
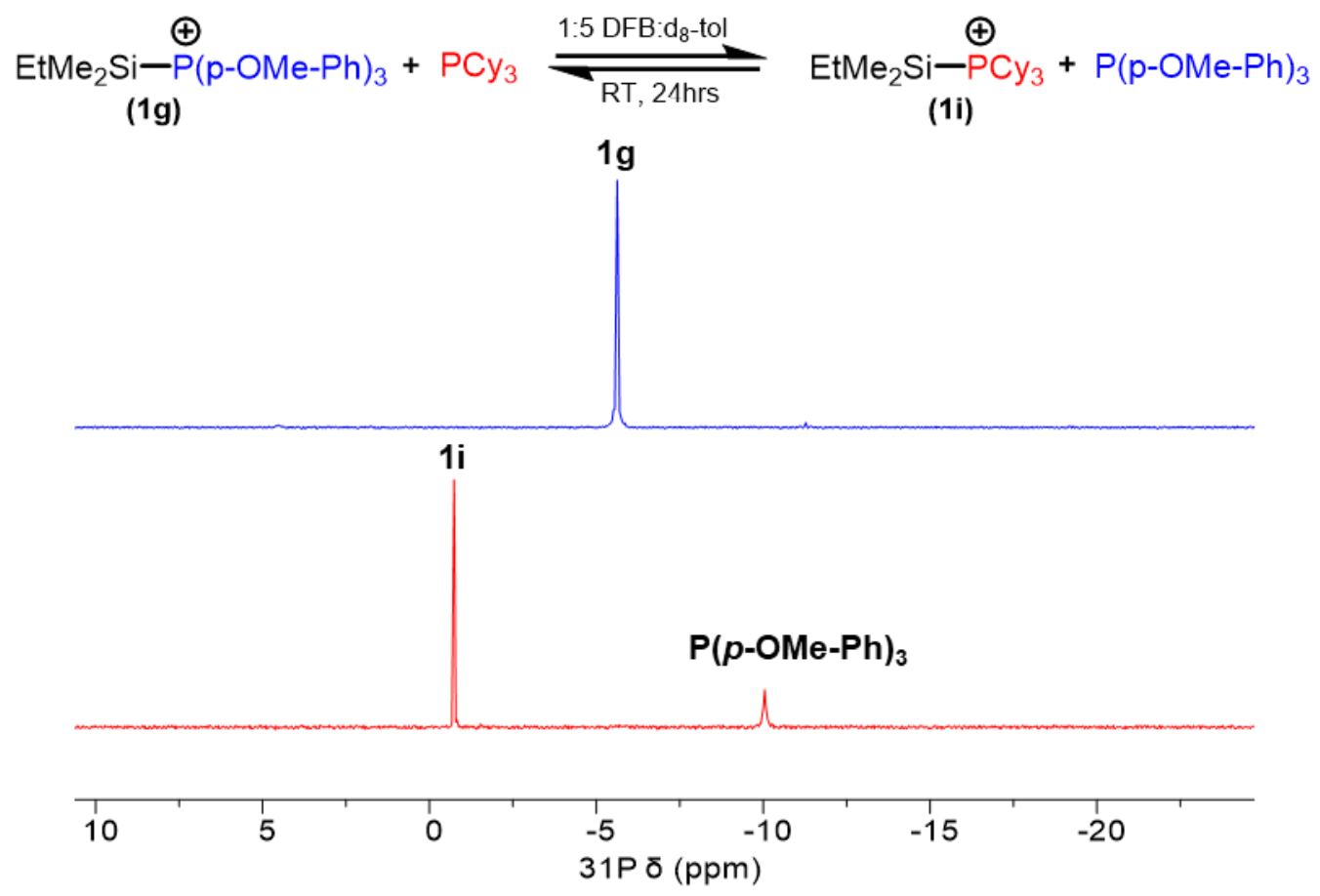

Figure S41. ${ }^{31} \mathrm{P}\left\{{ }^{1} \mathrm{H}\right\}$ NMR spectra detailing the stoichiometric transfer of $\mathrm{Me}_{2} \mathrm{EtSi}^{+}$from $\left[\mathrm{Me}_{2} \mathrm{EtSi}-\right.$ $\left.\mathrm{P}(\mathrm{p}-\mathrm{OMe}-\mathrm{Ph})_{3}\right]^{+}\left(-5.61 \mathrm{ppm}\right.$, blue spectra) to $\left[\mathrm{Me}_{2} \mathrm{EtSi}^{\left.-P C y_{3}\right]^{+}}(-0.74 \mathrm{ppm}\right.$, red spectra). At equilibrium no visible $\left[\mathrm{Me}_{2} \mathrm{EtSi}-\mathrm{P}(p-\mathrm{OMe}-\mathrm{Ph})_{3}\right]^{+}$is observed indicating a stoichiometric transfer. Assessment of detection limits indicate that the equilibrium for transfer is $>20$. 


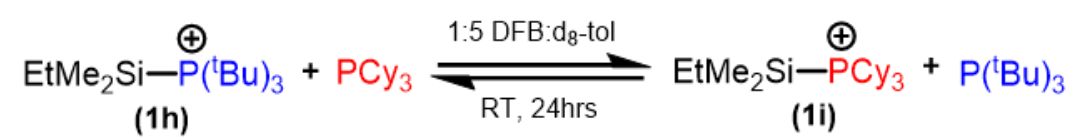

$1 \mathrm{~h}$

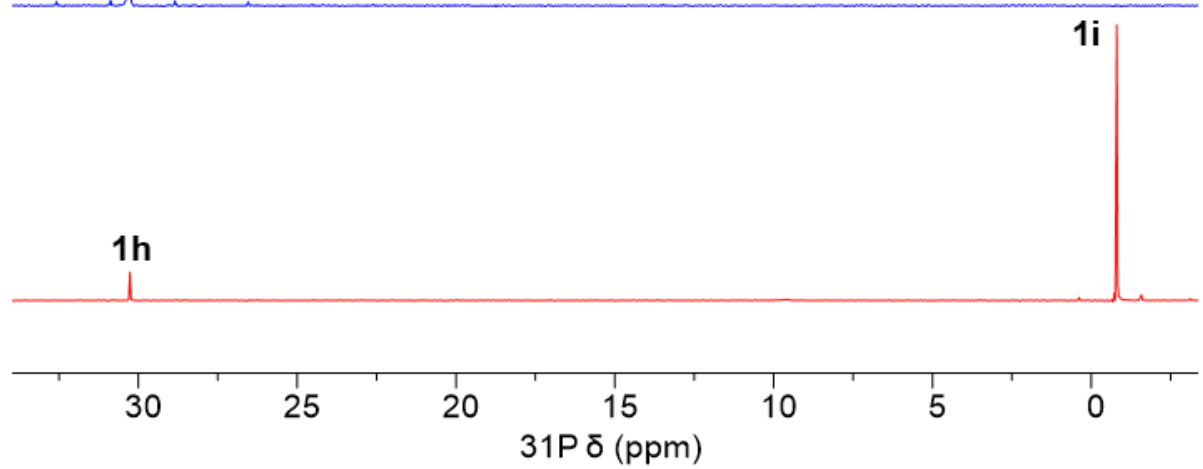

Figure S42. ${ }^{31} \mathrm{P}\left\{{ }^{1} \mathrm{H}\right\}$ NMR spectra detailing the stoichiometric transfer of $\mathrm{Me}_{2} \mathrm{EtSi}^{+}$from $\left[\mathrm{Me}_{2} \mathrm{EtSi}-\right.$ $\left.\mathrm{P}\left({ }^{\mathrm{t}} \mathrm{Bu}\right)_{3}\right]^{+}\left(30.5 \mathrm{ppm}\right.$, blue spectra) to $\left[\mathrm{Me}_{2} \mathrm{EtSi}^{-} \mathrm{PCy}_{3}\right]^{+}(-0.74 \mathrm{ppm}$, red spectra). At equilibrium $\left[\mathrm{Me}_{2} \mathrm{EtSi}-\mathrm{P}\left({ }^{\mathrm{t}} \mathrm{Bu}\right)_{3}\right]^{+}$is observed indicating a partial transfer. Assessment of detection limits indicate that the equilibrium for transfer is 8.1.
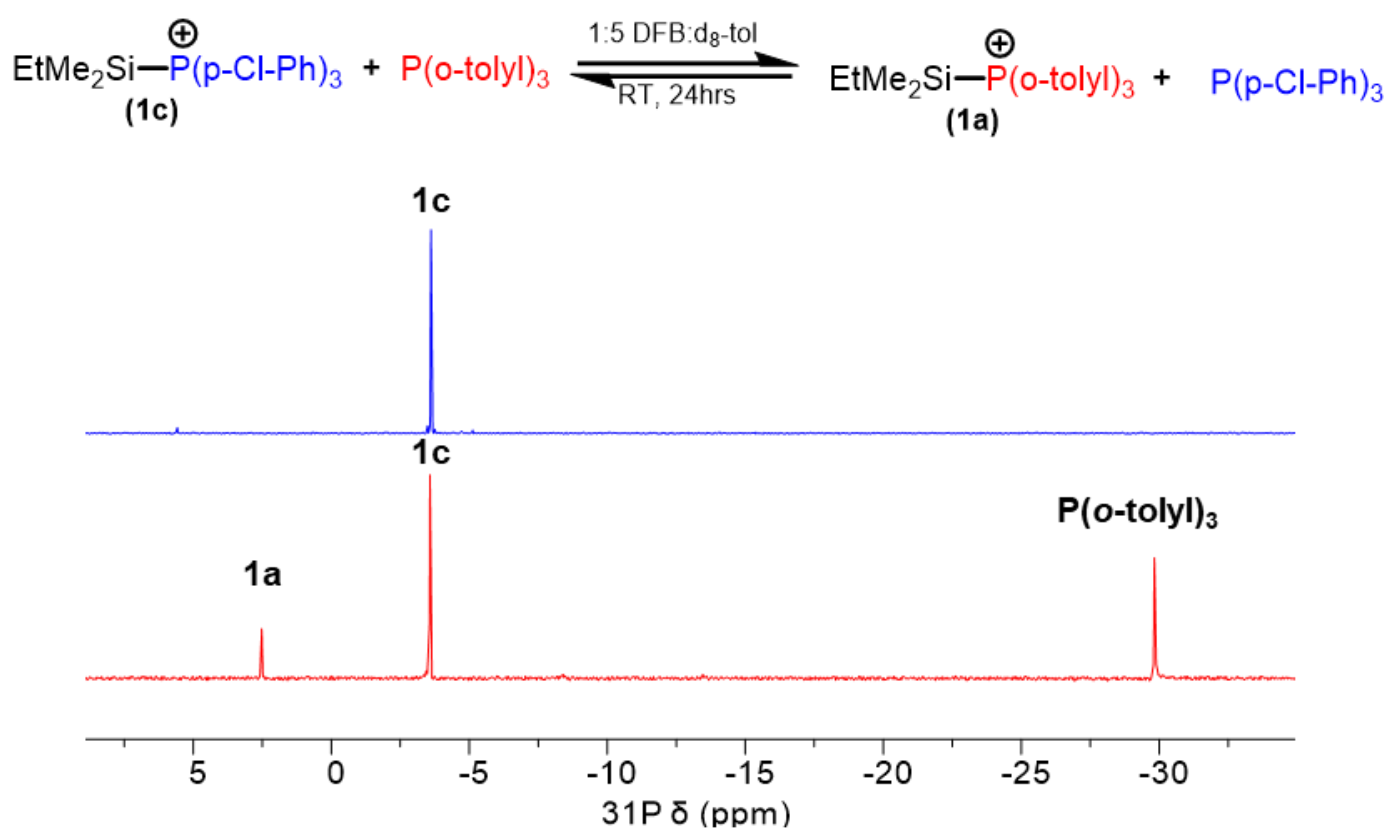

Figure S43. ${ }^{31} \mathrm{P}\left\{{ }^{1} \mathrm{H}\right\}$ NMR spectra detailing the stoichiometric transfer of $\mathrm{Me}_{2} \mathrm{EtSi}^{+}$from $\left[\mathrm{Me}_{2} \mathrm{EtSi}-\mathrm{P}(\mathrm{p}-\mathrm{Cl}-\mathrm{Ph})_{3}\right]^{+}\left(-3.61 \mathrm{ppm}\right.$, blue spectra) to $\left[\mathrm{Me}_{2} \mathrm{EtSi}-\mathrm{P}(0-\text { tolyl })_{3}\right]^{+}(2.52 \mathrm{ppm}$, red spectra). At equilibrium $\left[\mathrm{Me}_{2} \mathrm{EtSi}-\mathrm{P}(\mathrm{p}-\mathrm{Cl}-\mathrm{Ph})_{3}\right]^{+}$is observed indicating a partial transfer. Assessment of detection limits indicate that the equilibrium for transfer is 0.10 . 

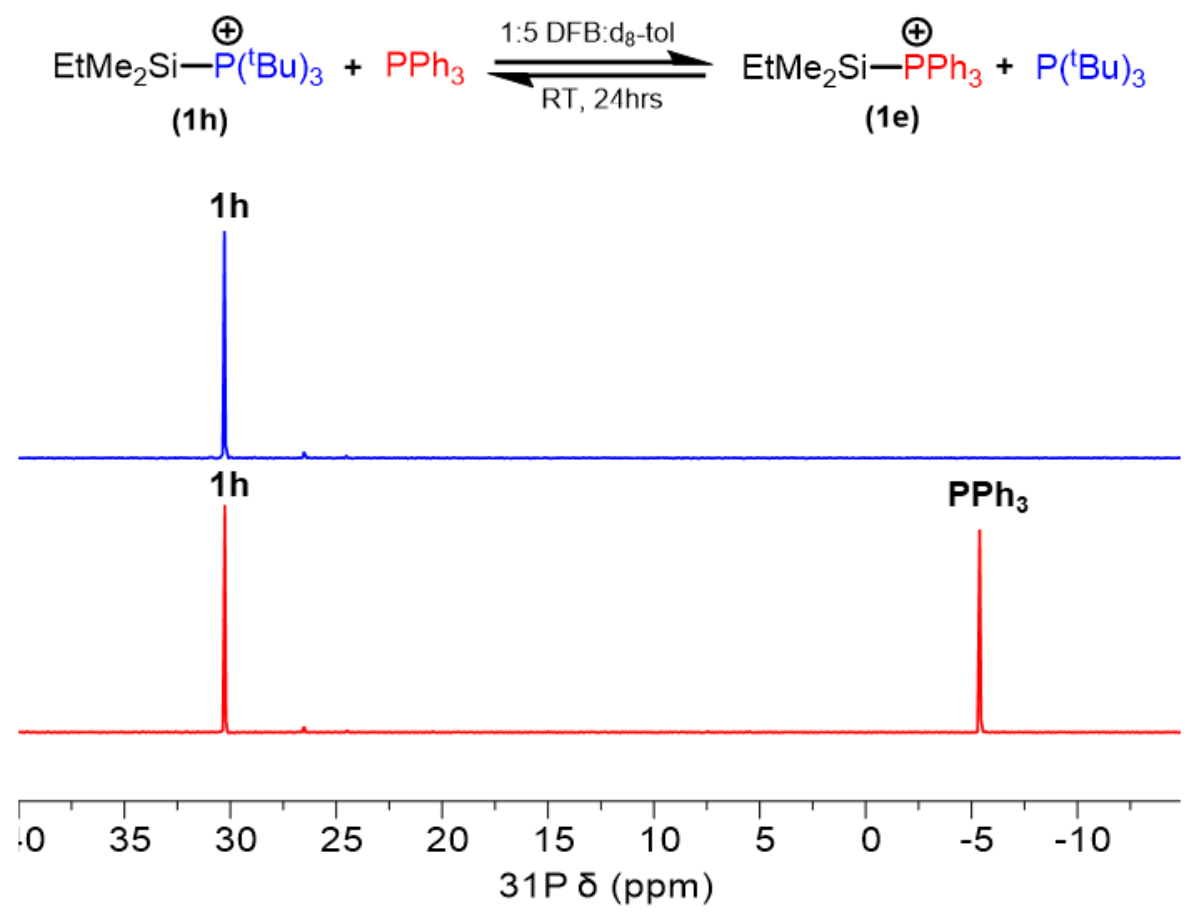

Figure S44. ${ }^{31} \mathrm{P}\left\{{ }^{1} \mathrm{H}\right\}$ NMR spectra detailing the stoichiometric transfer of $\mathrm{Me}_{2} \mathrm{EtSi}^{+}$from $\left[\mathrm{Me}_{2} \mathrm{EtSi}-\mathrm{P}\left({ }^{\mathrm{t} B u}\right)_{3}\right]^{+}\left(30.5 \mathrm{ppm}\right.$, blue spectra) to $\left[\mathrm{Me}_{2} \mathrm{EtSi}-\mathrm{PPh}_{3}\right]^{+}(-3.15 \mathrm{ppm}$, red spectra). At equilibrium only $\left[\mathrm{Me}_{2} \mathrm{EtSi}-\mathrm{P}\left({ }^{\mathrm{t}} \mathrm{Bu}\right)_{3}\right]^{+}$is observed indicating little to no transfer. Assessment of detection limits indicate that the equilibrium for transfer is $<0.10$.

Table S1. Spectroscopic, computational, and steric parameters for the studied $\mathrm{Me}_{2} \mathrm{EtSi}-\mathrm{PR}_{3}{ }^{+}$complexes

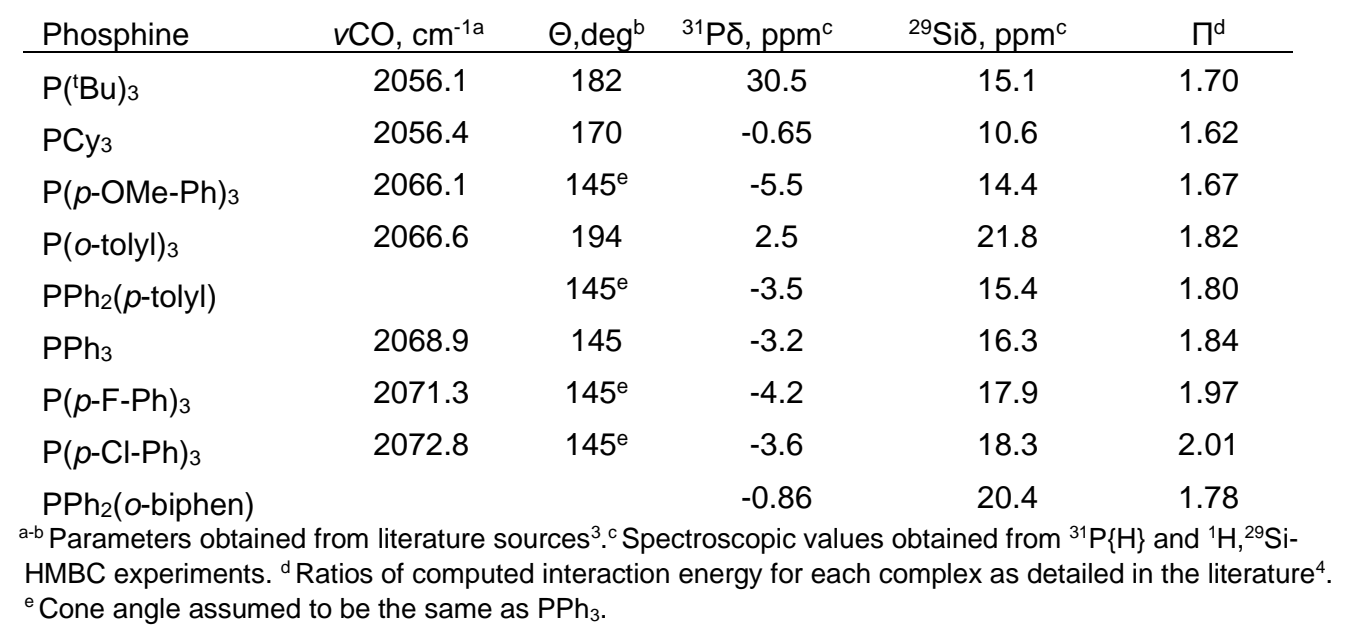


Table S2. Spectroscopic and Computational parameters for $\mathrm{R}_{3} \mathrm{Si}-\mathrm{PPh}_{3}{ }^{+}$Complexes

\begin{tabular}{lccc} 
Silane & ${ }^{31} \mathrm{P} \delta(\mathrm{ppm})^{\mathrm{a}}$ & ${ }^{29} \mathrm{Si}(\mathrm{ppm})^{\mathrm{a}}$ & $\Pi^{\mathrm{b}}$ \\
\hline $\mathrm{Me}_{2} \mathrm{EtSiH}$ & -3.2 & 16.4 & 1.87 \\
$\mathrm{Et}_{3} \mathrm{SiH}(\mathrm{TES})$ & -3.4 & 19.0 & 1.89 \\
$\left.{ }^{(} \mathrm{Pr}\right)_{3} \mathrm{SiH}(\mathrm{TIPS})$ & -1.7 & 25.9 & 1.93 \\
$\mathrm{MeEt}_{2} \mathrm{SiH}$ & -3.2 & 17.4 & 1.84 \\
${ }^{\mathrm{t} B u M e}{ }_{2} \mathrm{SiH}$ & -1.8 & 20.8 & 1.89
\end{tabular}

aspectroscopic values obtained from ${ }^{31} \mathrm{P}\{\mathrm{H}\}$ and ${ }^{1} \mathrm{H},{ }^{29} \mathrm{Si}-\mathrm{HMBC}$ experiments. ${ }^{\mathrm{b}}$ Ratios of computed interaction energy for each complex as detailed in the literature. ${ }^{3}$

Table S3. Computed Mulliken Charges for $\mathrm{R}_{3} \mathrm{Si}-\mathrm{PR}_{3}{ }^{+}$Complexes

\begin{tabular}{ccccc} 
entry & Lewis Base & $\mathrm{SiR}_{3}$ & Si Mulliken Charge & $\mathrm{P}$ Mulliken Charge \\
\hline 1 & $\mathrm{PPh}_{2}(o-$ biphen $)$ & $\mathrm{SiMe}_{2} \mathrm{Et}$ & 1.090 & 0.8822 \\
2 & $\mathrm{P}(o-\mathrm{Tol})_{3}$ & $\mathrm{SiMe}_{2} \mathrm{Et}$ & 1.265 & 0.6475 \\
3 & $\mathrm{PCy}_{3}$ & $\mathrm{SiMe}_{2} \mathrm{Et}$ & 1.035 & 0.8829 \\
4 & $\mathrm{P}(t-\mathrm{Bu})_{3}$ & $\mathrm{SiMe}_{2} \mathrm{Et}$ & 0.985 & 0.9359 \\
5 & $\mathrm{P}(p-\mathrm{OMe}-\mathrm{Ph})_{3}$ & $\mathrm{SiMe}_{2} \mathrm{Et}$ & 1.066 & 0.7985 \\
6 & $\mathrm{PPh}_{2}(p-\mathrm{Tol})$ & $\mathrm{SiMe}_{2} \mathrm{Et}$ & 1.070 & 0.8203 \\
7 & $\mathrm{PPh}_{3}$ & $\mathrm{SiMe}_{2} \mathrm{Et}$ & 1.070 & 0.8213 \\
8 & $\mathrm{P}(p-\mathrm{F}-\mathrm{Ph})_{3}$ & $\mathrm{SiMe}_{2} \mathrm{Et}$ & 1.071 & 0.8082 \\
9 & $\mathrm{P}(p-\mathrm{Cl} \mathrm{Ph})_{3}$ & $\mathrm{SiMe}_{2} \mathrm{Et}$ & 1.068 & 0.8123 \\
10 & $\mathrm{PPh}_{3}$ & $\mathrm{SiEt}_{2} \mathrm{Me}$ & 1.107 & 0.8074 \\
11 & $\mathrm{PPh}_{3}$ & $\mathrm{SiEt}_{3}$ & 1.130 & 0.8371 \\
12 & $\mathrm{PPh}_{3}$ & $\mathrm{SiBuMe}_{2}$ & 1.128 & 0.8154 \\
13 & $\mathrm{PPh}_{3}$ & $\mathrm{SiPr}_{3}$ & 1.217 & 0.7374
\end{tabular}


PARAMETER COMPARISONS

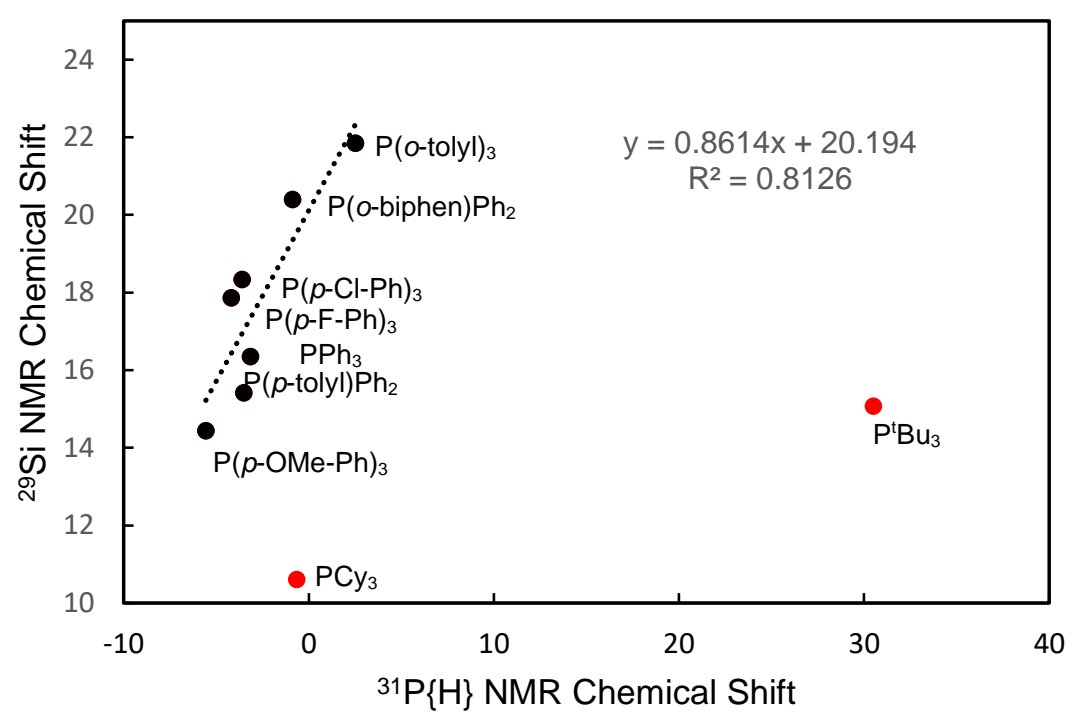

Figure S45. Plot of ${ }^{31} \mathrm{P}\{\mathrm{H}\}$ NMR versus ${ }^{29} \mathrm{Si}$ NMR for a family of $\mathrm{Me}_{2} \mathrm{EtSi}-\mathrm{PR}_{3}{ }^{+}$ complexes. Poor correlations are observed when the bulky trialkyl silylphosphoniums, $\mathrm{P}\left({ }^{\mathrm{t}} \mathrm{Bu}\right)_{3}$ and $\mathrm{PCy}_{3}$, are included in the fit.

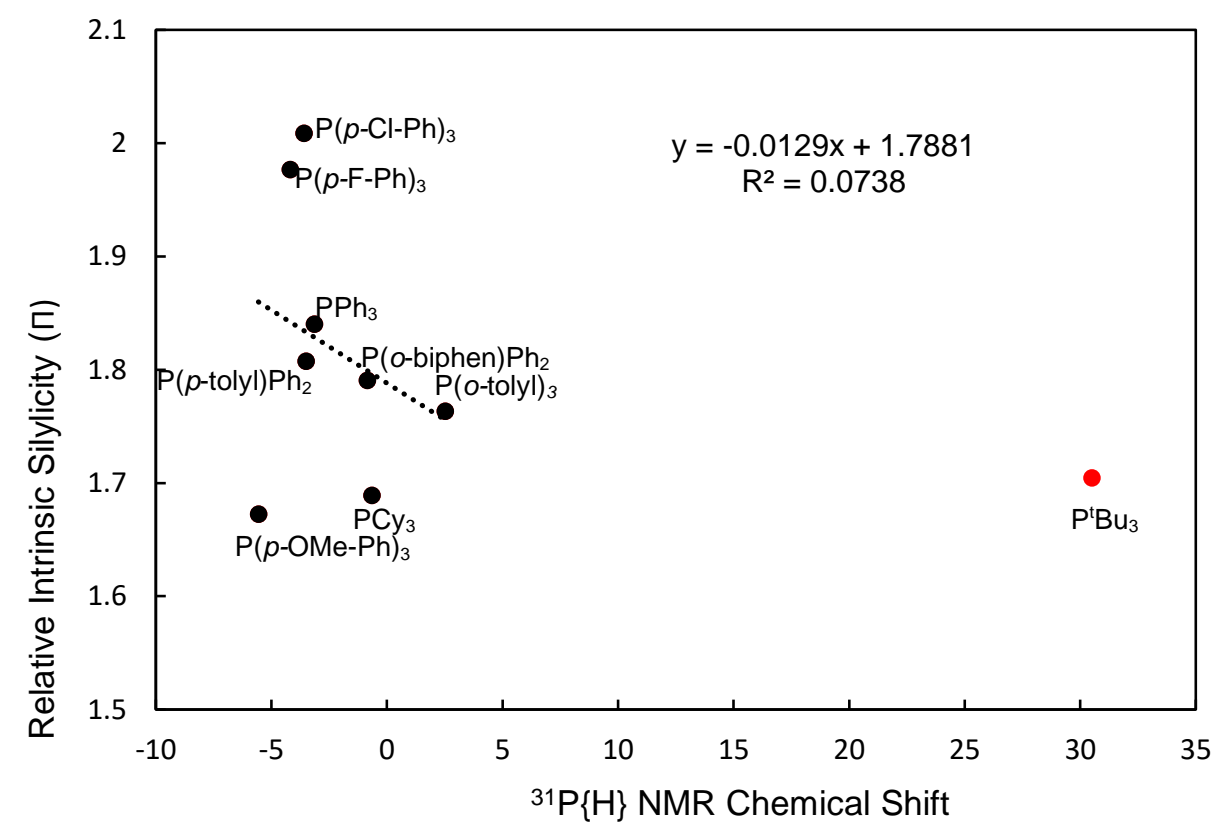

Figure S46. A plot of ${ }^{31} \mathrm{P}\{\mathrm{H}\} \mathrm{NMR}$ chemical shift versus relative intrinsic silylicity $(\Pi)$ of a family of $\mathrm{Me}_{2} \mathrm{EtSi}-\mathrm{PR}_{3}{ }^{+}$complexes show no correlation even when excluding the outlier, EtMe $2 \mathrm{Si}-\mathrm{P}\left({ }^{\mathrm{B}} \mathrm{Bu}\right)_{3}$. 


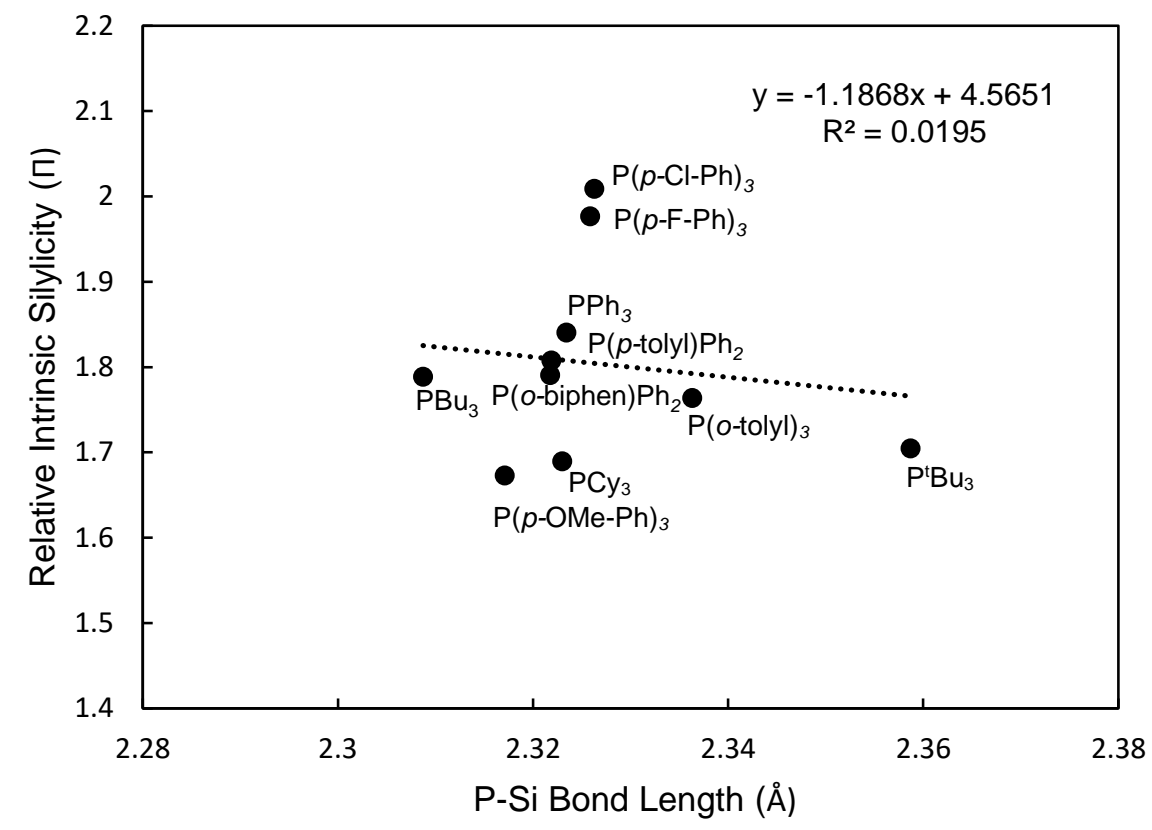

Figure S47. A plot of computed P-Si bond length versus relative intrinsic silylicity $(\Pi)$ of a family of $\mathrm{Me}_{2} \mathrm{EtSi}-\mathrm{PR}_{3}{ }^{+}$complexes show no correlation.

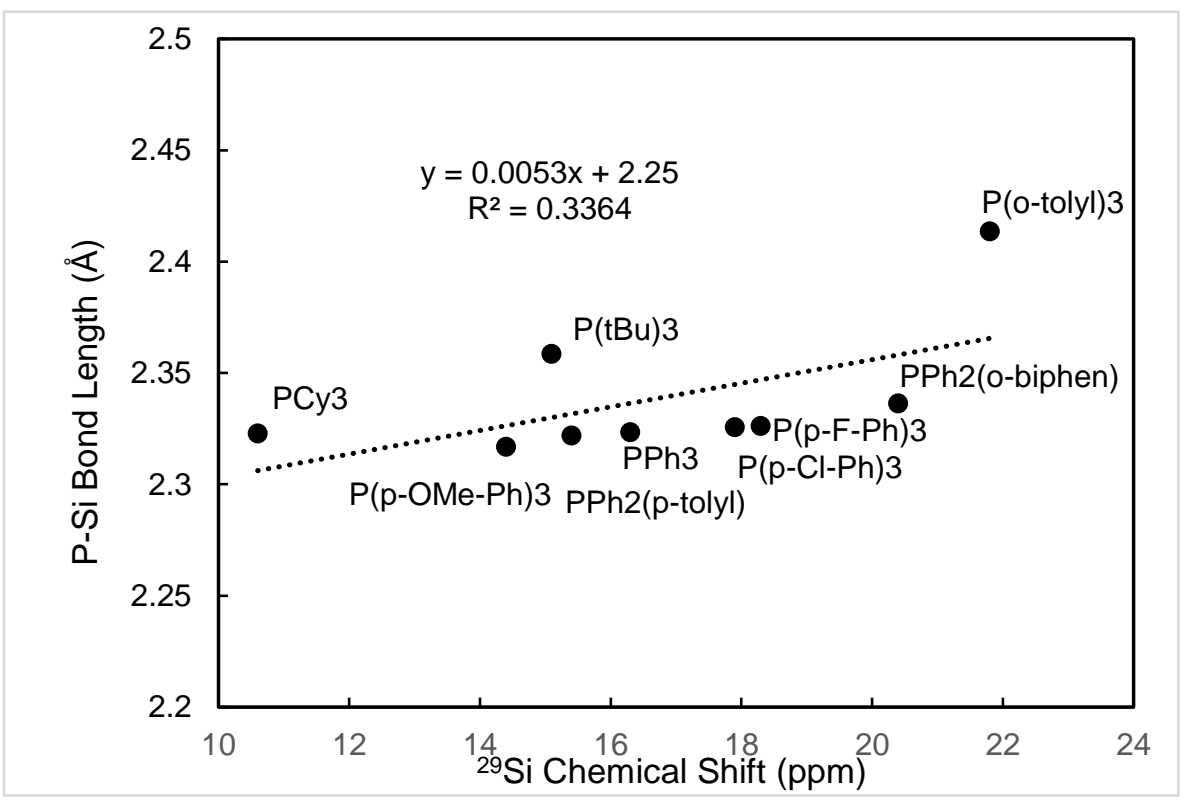

Figure S48. A plot of experimental ${ }^{29} \mathrm{Si}$ NMR chemical shift versus computed $\mathrm{P}-$ $\mathrm{Si}$ bond length of a family of $\mathrm{Me}_{2} \mathrm{EtSi}-\mathrm{PR}_{3}{ }^{+}$complexes show poor correlation. 


\section{COMPUTATIONAL DETAILS}

Computational Methods: Calculations were carried out with the Gaussian 09 program package, revision E.01. Geometry optimizations and frequency calculations were performed in the gas phase with no counteranion present using the dispersion corrected (GD3BJ) PBE1PBE function with the following basis sets: $6-311 \mathrm{G}^{*}(\mathrm{C}, \mathrm{H}, \mathrm{Si})$ and $6-311+\mathrm{G}^{*}(\mathrm{O}, \mathrm{F}, \mathrm{Cl}, \mathrm{S}, \mathrm{P})$. Basis set superposition error (BSSE) corrected interaction energies (complexation energies) were obtained by computing the counterpoise corrections of the Lewis base-silylium adducts, with the silylium moiety assigned a charge of +1 and the Lewis base assigned a charge of $q-1$, where $q$ is the overall charge of the adduct.

Table S4: Selected Computational Parameters

\begin{tabular}{|c|c|c|c|c|c|}
\hline Lewis Base & $\mathrm{SiR}_{3}{ }^{+}$ & $\begin{array}{l}\text { Lowest } \\
\text { frequency } \\
\left(\mathrm{cm}^{-1}\right)\end{array}$ & $\Delta \mathbf{G}$ (Hartree) & $\begin{array}{l}\text { Corrected } E_{\text {int }} \\
\quad(\mathrm{kcal} / \mathrm{mol})\end{array}$ & $\Pi^{\mathrm{a}}$ \\
\hline $\mathrm{PPh}_{3}$ & $\mathrm{Et}_{2} \mathrm{Me}$ & 15.2126 & -1522.529611 & -92.29 & 1.84 \\
\hline $\mathrm{PPh}_{3}$ & ${ }^{t} \mathrm{BuMe}_{2}$ & 16.4508 & -1561.776878 & -89.87 & 1.89 \\
\hline $\mathrm{PPh}_{3}$ & $\mathrm{Et}_{3}$ & 12.7584 & -1561.772093 & -89.39 & 1.89 \\
\hline $\mathrm{PPh}_{3}$ & $\mathrm{Si}^{i} \mathrm{Pr}_{3}$ & 26.2885 & -1679.504028 & -84.9 & 1.93 \\
\hline $\mathrm{PPh}_{3}$ & $\mathrm{EtMe}_{2}$ & 14.4506 & -1483.284396 & -93.84 & 1.84 \\
\hline $\mathrm{PPh}_{3}$ & $\mathrm{EtMe}_{2}$ & 18.91 & -1483.285926 & -92.33 & 1.87 \\
\hline OTf & $\mathrm{Et}_{2} \mathrm{Me}$ & 14.8915 & -1448.396258 & -170.25 & \\
\hline OTf & $\mathrm{EtMe}_{2}$ & 29.3571 & -1409.151603 & -172.42 & \\
\hline OTf & ${ }^{t} \mathrm{BuMe}_{2}$ & 25.7364 & -1487.649275 & -170.04 & \\
\hline OTf & $\mathrm{Et}_{3}$ & 15.3963 & -1487.641116 & -168.65 & \\
\hline OTf & $\mathrm{Si}^{i} \mathrm{Pr}_{3}$ & 17.4533 & -1605.37665 & -163.98 & \\
\hline $\mathrm{P}(4-\mathrm{Cl}-\mathrm{Ph})_{3}$ & $\mathrm{EtMe}_{2}$ & 11.8029 & -2861.709056 & -85.96 & 2.01 \\
\hline $\mathrm{P}(4-\mathrm{F}-\mathrm{Ph})_{3}$ & $\mathrm{EtMe}_{2}$ & 11.9076 & -1780.847004 & -87.36 & 1.97 \\
\hline $\begin{array}{c}\mathrm{P}(4-\mathrm{OMe}- \\
\mathrm{Ph})_{3}\end{array}$ & $\mathrm{EtMe}_{2}$ & 12.9459 & -1826.503649 & -103.24 & 1.67 \\
\hline $\begin{array}{c}\mathrm{PPh}_{2}(4-\mathrm{Me}- \\
\mathrm{Ph})\end{array}$ & $\mathrm{EtMe}_{2}$ & 14.9005 & -1522.541646 & -95.54 & 1.80 \\
\hline $\mathrm{PCy}_{3}$ & $\mathrm{EtMe}_{2}$ & 38.9801 & -1493.953204 & -106.14 & 1.62 \\
\hline $\mathrm{P}^{t} \mathrm{Bu}_{3}$ & $\mathrm{EtMe}_{2}$ & 56.138 & -1261.960157 & -101.3 & 1.70 \\
\hline $\begin{array}{l}\mathrm{PPh}_{2}\left(\mathrm{O}^{-}\right. \\
\text {Biphen })\end{array}$ & $\mathrm{EtMe}_{2}$ & 29.0071 & -1714.05163 & -96.69 & 1.78 \\
\hline $\mathrm{P}(2-\mathrm{Me}-\mathrm{Ph})_{3}$ & $\mathrm{EtMe}_{2}$ & 31.8037 & -1602.424891 & -94.78 & 1.82 \\
\hline
\end{tabular}

${ }^{a} \Pi=E_{\text {int }} \operatorname{corrected}\left(\mathrm{TfO}-\mathrm{SiR}_{3}\right) / \mathrm{E}_{\text {int }} \operatorname{corrected}\left(\mathrm{R}_{3} \mathrm{P}-\mathrm{SiR}_{3}{ }^{{ }^{+}}\right)$ 


\section{X-Ray Crystallography Information-rds932}

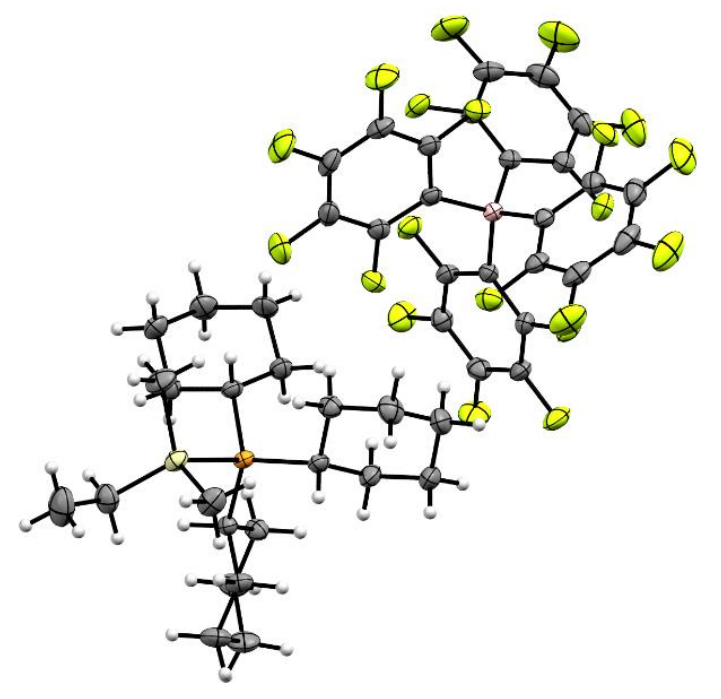

A colorless plate-like specimen of $\mathrm{C}_{46} \mathrm{H}_{44} \mathrm{BF}_{20} \mathrm{PSi}$, approximate dimensions $0.128 \mathrm{~mm} x$ $0.344 \mathrm{~mm} \times 0.415 \mathrm{~mm}$, was used for the X-ray crystallographic analysis. The X-ray intensity data were measured on a Bruker-Nonius X8 Kappa APEX II system equipped with a fine-focus sealed tube (MoKa, $\lambda=0.71073 \AA$ ) and a graphite monochromator.

The total exposure time was 9.79 hours. The frames were integrated with the Bruker SAINT software package using a narrow-frame algorithm. The integration of the data using a monoclinic unit cell yielded a total of 56416 reflections to a maximum $\theta$ angle of $27.56^{\circ}(0.77 \AA$ resolution), of which 10439 were independent (average redundancy 5.404 , completeness $\left.=99.6 \%, R_{\text {int }}=3.54 \%, R_{\text {sig }}=3.07 \%\right)$ and $8120(77.79 \%)$ were greater than $2 \sigma\left(F^{2}\right)$. The final cell constants of $\underline{a}=11.3597(6) \AA ⿻ 上 丨=33.848(2) \AA$, $\underline{c}=$ 11.7856(8) $\AA, \beta=91.517(2)^{\circ}$, volume $=4530.0(5) \AA^{3}$, are based upon the refinement of the $\mathrm{XYZ}$-centroids of 7169 reflections above $20 \sigma(\mathrm{I})$ with $3.623^{\circ}<2 \theta<56.38^{\circ}$. Data were corrected for absorption effects using the Multi-Scan method (SADABS). The ratio of minimum to maximum apparent transmission was 0.921 . The calculated minimum and maximum transmission coefficients (based on crystal size) are 0.6864 and 0.7456 .

The final anisotropic full-matrix least-squares refinement on $\mathrm{F}^{2}$ with 625 variables converged at $\mathrm{R} 1=4.99 \%$, for the observed data and $\mathrm{wR} 2=11.41 \%$ for all data. The goodness-of-fit was 1.118. The largest peak in the final difference electron density synthesis was $0.409 \mathrm{e}^{-/} / \AA^{3}$ and the largest hole was $-0.271 \mathrm{e}^{\mathrm{e}} / \AA^{3}$ with an RMS deviation of $0.052 \mathrm{e}^{-} / \AA^{3}$. On the basis of the final model, the calculated density was $1.535 \mathrm{~g} / \mathrm{cm}^{3}$ and $F(000), 2136 e^{-}$. 


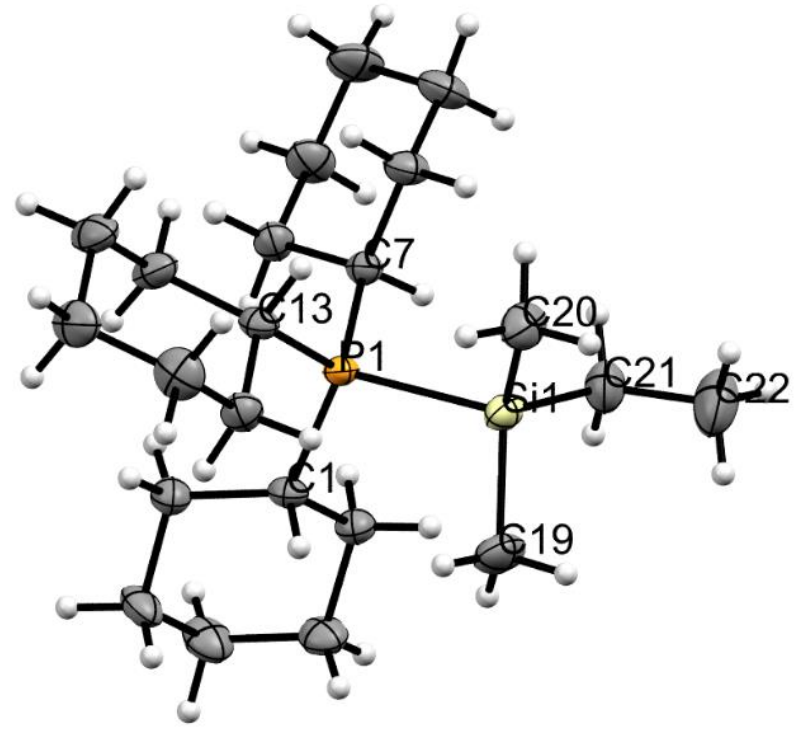

Figure S49. Significant atoms labeled for $\left[\mathrm{EtMe}_{2} \mathrm{Si}_{-}-\mathrm{PCy}_{3}\right]^{+} \mathrm{X}$-Ray Crystal Structure Table S5. Sample and crystal data for rds932.

$\begin{array}{lll}\text { Identification code } & \text { rds932 } & \\ \text { Chemical formula } & \mathrm{C}_{46} \mathrm{H}_{44} \mathrm{BF}_{20} \mathrm{PSi} & \\ \text { Formula weight } & 1046.68 \mathrm{~g} / \mathrm{mol} & \\ \text { Temperature } & 210(2) \mathrm{K} \\ \text { Wavelength } & 0.71073 \AA \\ \text { Crystal size } & 0.128 \times 0.344 \times 0.415 \mathrm{~mm} \\ \text { Crystal habit } & \text { colorless plate } & \\ \text { Crystal system } & \text { monoclinic } & \\ \text { Space group } & \mathrm{P} 121 / \mathrm{c} 1 & \\ \text { Unit cell dimensions } & \mathrm{a}=11.3597(6) \AA & \alpha=90^{\circ} \\ & \mathrm{b}=33.848(2) \AA & \beta=91.517(2)^{\circ} \\ & \mathrm{C}=11.7856(8) \AA & \mathrm{V}=90^{\circ} \\ \text { Volume } & 4530.0(5) \AA^{3} & \\ \text { Z } & 4 & \\ \text { Density (calculated) } & 1.535 \mathrm{~g} / \mathrm{cm}^{3} & \\ \text { Absorption coefficient } & 0.204 \mathrm{~mm}^{-1} & \\ \text { F(000) } & 2136 & \end{array}$


Table S6. Data collection and structure refinement for rds932.

\begin{tabular}{|c|c|}
\hline Diffractometer & Bruker-Nonius X8 Kappa APEX II \\
\hline Radiation source & fine-focus sealed tube (MoK $\alpha, \lambda=0.71073 \AA$ ) \\
\hline Theta range for data collection & 1.79 to $27.56^{\circ}$ \\
\hline Index ranges & $-14<=h<=13,-44<=k<=43,-15<=\mid<=13$ \\
\hline Reflections collected & 56416 \\
\hline Independent reflections & $10439[\mathrm{R}(\mathrm{int})=0.0354]$ \\
\hline Coverage of independent reflections & $99.60 \%$ \\
\hline Absorption correction & Multi-Scan \\
\hline Max. and min. transmission & 0.7456 and 0.6864 \\
\hline Refinement method & Full-matrix least-squares on $\mathrm{F}^{2}$ \\
\hline Refinement program & SHELXL-2018/3 (Sheldrick, 2018) \\
\hline Function minimized & $\Sigma w\left(F_{o}^{2}-F_{c}^{2}\right)^{2}$ \\
\hline Data / restraints / parameters & 10439 / 0 / 625 \\
\hline Goodness-of-fit on $F^{2}$ & 1.118 \\
\hline$\Delta / \sigma_{\max }$ & 0.001 \\
\hline Final $\mathbf{R}$ indices & $\begin{array}{ll}8120 \text { data; } I>2 \sigma(I) & R 1=0.0499, w R 2=0.1070 \\
\text { all data } & R 1=0.0696, w R 2=0.1141\end{array}$ \\
\hline Weighting scheme & $\begin{array}{l}\mathrm{w}=1 /\left[\sigma^{2}\left(\mathrm{~F}_{\mathrm{o}}^{2}\right)+(0.0329 \mathrm{P})^{2}+4.3519 \mathrm{P}\right] \\
\text { where } \mathrm{P}=\left(\mathrm{F}_{\mathrm{o}}^{2}+2 \mathrm{~F}_{\mathrm{c}}^{2}\right) / 3\end{array}$ \\
\hline Largest diff. peak and hole & 0.409 and $-0.271 \mathrm{e}^{-3}$ \\
\hline R.M.S. deviation from mean & $0.052 \mathrm{e}^{-3}$ \\
\hline
\end{tabular}

Table S7. Atomic coordinates and equivalent isotropic atomic displacement parameters $\left(\AA^{2}\right)$ for rds932.

$\mathrm{U}(\mathrm{eq})$ is defined as one third of the trace of the orthogonalized $\mathrm{U}_{\mathrm{ij}}$ tensor.

$\begin{array}{lllll} & \mathrm{x} / \mathrm{a} & \mathrm{y} / \mathrm{b} & \mathrm{z} / \mathrm{c} & \mathrm{U}(\mathrm{eq}) \\ \text { P1 } & 0.62255(4) & 0.37246(2) & 0.43974(5) & 0.01930(12) \\ \text { Si1 } & 0.62408(5) & 0.32063(2) & 0.31139(5) & 0.02642(14) \\ \text { C1 } & 0.48403(18) & 0.39936(6) & 0.41415(18) & 0.0231(4) \\ \text { C2 } & 0.48416(19) & 0.41898(7) & 0.29670(19) & 0.0269(5) \\ \text { C3 } & 0.3627(2) & 0.43613(8) & 0.2663(2) & 0.0347(6) \\ \text { C4 } & 0.3230(2) & 0.46450(8) & 0.3560(2) & 0.0400(6) \\ \text { C5 } & 0.3225(2) & 0.44482(8) & 0.4720(2) & 0.0360(6) \\ \text { C6 } & 0.4439(2) & 0.42820(7) & 0.5054(2) & 0.0293(5) \\ \text { C7 } & 0.75266(17) & 0.40262(6) & 0.41155(18) & 0.0216(4)\end{array}$




\begin{tabular}{|c|c|c|c|c|}
\hline C8 & $0.7605(2)$ & $0.44347(6)$ & $0.4674(2)$ & $0.0273(5)$ \\
\hline C9 & $0.8691(2)$ & $0.46518(7)$ & $0.4269(2)$ & $0.0362(6)$ \\
\hline C10 & $0.9813(2)$ & $0.44158(8)$ & $0.4482(3)$ & $0.0417(6)$ \\
\hline C11 & $0.9725(2)$ & $0.40075(8)$ & $0.3950(2)$ & $0.0366(6)$ \\
\hline C12 & $0.86544(18)$ & $0.37854(7)$ & $0.4367(2)$ & $0.0293(5)$ \\
\hline C13 & $0.63002(18)$ & $0.35081(6)$ & $0.58209(18)$ & $0.0236(4)$ \\
\hline C14 & $0.5189(2)$ & $0.32597(7)$ & $0.6010(2)$ & $0.0283(5)$ \\
\hline C15 & $0.5250(2)$ & $0.30539(8)$ & $0.7157(2)$ & $0.0390(6)$ \\
\hline C16 & $0.5475(2)$ & $0.33472(9)$ & $0.8113(2)$ & $0.0399(6)$ \\
\hline C17 & $0.6593(2)$ & $0.35786(9)$ & $0.7935(2)$ & $0.0378(6)$ \\
\hline C18 & $0.6545(2)$ & $0.37975(7)$ & $0.6802(2)$ & $0.0319(5)$ \\
\hline C19 & $0.4680(2)$ & $0.30745(8)$ & $0.2788(2)$ & $0.0408(6)$ \\
\hline C20 & $0.7056(2)$ & $0.27909(7)$ & $0.3812(2)$ & $0.0380(6)$ \\
\hline $\mathrm{C} 21$ & $0.6998(3)$ & $0.33809(8)$ & $0.1818(2)$ & $0.0396(6)$ \\
\hline $\mathrm{C} 22$ & $0.7150(3)$ & $0.30503(10)$ & $0.0961(3)$ & $0.0605(9)$ \\
\hline $\mathrm{F} 1$ & $0.27226(12)$ & $0.36868(4)$ & $0.67642(12)$ & $0.0370(3)$ \\
\hline $\mathrm{F} 2$ & $0.20758(14)$ & $0.32706(5)$ & $0.49699(13)$ & $0.0500(4)$ \\
\hline F3 & $0.00569(15)$ & $0.28313(5)$ & $0.49597(14)$ & $0.0539(4)$ \\
\hline $\mathrm{F} 4$ & $0.87056(13)$ & $0.28281(5)$ & $0.68268(14)$ & $0.0489(4)$ \\
\hline F5 & $0.93359(11)$ & $0.32505(4)$ & $0.86522(12)$ & $0.0357(3)$ \\
\hline F6 & $0.30788(12)$ & $0.31311(4)$ & $0.89702(12)$ & $0.0355(3)$ \\
\hline F7 & $0.34774(15)$ & $0.25557(4)$ & $0.04680(15)$ & $0.0532(4)$ \\
\hline F8 & $0.21304(17)$ & $0.24888(5)$ & $0.23329(15)$ & $0.0606(5)$ \\
\hline F9 & $0.02610(17)$ & $0.29864(6)$ & $0.25627(14)$ & $0.0623(5)$ \\
\hline F10 & $0.98207(13)$ & $0.35488(5)$ & $0.10806(13)$ & $0.0431(4)$ \\
\hline F11 & $0.36682(12)$ & $0.37437(4)$ & $0.04895(11)$ & $0.0342(3)$ \\
\hline F12 & $0.54174(12)$ & $0.42444(5)$ & $0.03063(13)$ & $0.0421(4)$ \\
\hline F13 & $0.53240(13)$ & $0.48375(5)$ & $0.87481(14)$ & $0.0469(4)$ \\
\hline F14 & $0.34174(14)$ & $0.49006(4)$ & $0.73244(13)$ & $0.0456(4)$ \\
\hline F15 & $0.16591(12)$ & $0.43835(4)$ & $0.74232(12)$ & $0.0353(3)$ \\
\hline F16 & $0.12568(13)$ & $0.41995(5)$ & $0.10697(12)$ & $0.0394(3)$ \\
\hline F17 & $0.97029(17)$ & $0.47500(5)$ & $0.16232(14)$ & $0.0557(5)$ \\
\hline F18 & $0.79052(16)$ & $0.49453(5)$ & $0.01562(17)$ & $0.0635(5)$ \\
\hline F19 & $0.77418(14)$ & $0.45849(5)$ & $0.80906(16)$ & $0.0546(5)$ \\
\hline F20 & $0.92683(12)$ & $0.40446(4)$ & $0.75166(12)$ & $0.0366(3)$ \\
\hline $\mathrm{C} 23$ & $0.10479(18)$ & $0.35003(6)$ & $0.78124(19)$ & $0.0236(4)$ \\
\hline $\mathrm{C} 24$ & $0.17009(19)$ & $0.34853(7)$ & $0.6838(2)$ & $0.0269(5)$ \\
\hline C25 & $0.1387(2)$ & $0.32678(8)$ & $0.5884(2)$ & $0.0333(5)$ \\
\hline C26 & $0.0379(2)$ & $0.30464(7)$ & $0.5874(2)$ & $0.0349(6)$ \\
\hline $\mathrm{C} 27$ & $0.9704(2)$ & $0.30439(7)$ & $0.6822(2)$ & $0.0322(5)$ \\
\hline C28 & $0.00530(19)$ & $0.32646(7)$ & $0.7754(2)$ & $0.0277(5)$ \\
\hline C29 & $0.14762(18)$ & $0.33906(6)$ & $0.99673(19)$ & $0.0250(5)$ \\
\hline C30 & $0.23566(19)$ & $0.31089(7)$ & $0.9855(2)$ & $0.0277(5)$ \\
\hline
\end{tabular}




$\begin{array}{lllll}\text { C31 } & 0.2578(2) & 0.28072(7) & 0.0618(2) & 0.0353(6) \\ \text { C32 } & 0.1898(2) & 0.27722(7) & 0.1551(2) & 0.0407(7) \\ \text { C33 } & 0.0973(2) & 0.30265(8) & 0.1678(2) & 0.0390(6) \\ \text { C34 } & 0.0781(2) & 0.33219(7) & 0.0887(2) & 0.0321(5) \\ \text { C35 } & 0.25549(18) & 0.40259(6) & 0.89418(18) & 0.0222(4) \\ \text { C36 } & 0.35465(19) & 0.40180(6) & 0.96588(18) & 0.0235(4) \\ \text { C37 } & 0.44741(19) & 0.42787(7) & 0.95952(19) & 0.0278(5) \\ \text { C38 } & 0.4444(2) & 0.45756(7) & 0.8811(2) & 0.0300(5) \\ \text { C39 } & 0.3474(2) & 0.46043(7) & 0.8083(2) & 0.0287(5) \\ \text { C40 } & 0.25763(18) & 0.43346(7) & 0.81657(19) & 0.0259(5) \\ \text { C41 } & 0.03214(18) & 0.40718(6) & 0.92918(19) & 0.0240(4) \\ \text { C42 } & 0.0375(2) & 0.42798(7) & 0.0307(2) & 0.0290(5) \\ \text { C43 } & 0.9585(2) & 0.45644(8) & 0.0615(2) & 0.0374(6) \\ \text { C44 } & 0.8677(2) & 0.46641(8) & 0.9876(3) & 0.0406(6) \\ \text { C45 } & 0.8596(2) & 0.44806(7) & 0.8849(2) & 0.0353(6) \\ \text { C46 } & 0.94085(19) & 0.41962(7) & 0.8571(2) & 0.0277(5) \\ \text { B1 } & 0.1354(2) & 0.37456(7) & 0.9002(2) & 0.0222(5)\end{array}$

Table S8. Bond lengths ( $(\AA)$ for rds 932 .

$\begin{array}{llll}\text { P1-C13 } & 1.831(2) & \text { P1-C7 } & 1.834(2) \\ \text { P1-C1 } & 1.836(2) & \text { P1-Si1 } & 2.3169(8) \\ \text { Si1-C19 } & 1.858(2) & \text { Si1-C20 } & 1.863(2) \\ \text { Si1-C21 } & 1.868(3) & \text { C1-C6 } & 1.531(3) \\ \text { C1-C2 } & 1.535(3) & \text { C1-H1 } & 0.99 \\ \text { C2-C3 } & 1.530(3) & \text { C2-H2A } & 0.98 \\ \text { C2-H2B } & 0.98 & \text { C3-C4 } & 1.506(4) \\ \text { C3-H3A } & 0.98 & \text { C3-H3B } & 0.98 \\ \text { C4-C5 } & 1.521(4) & \text { C4-H4A } & 0.98 \\ \text { C4-H4B } & 0.98 & \text { C5-C6 } & 1.531(3) \\ \text { C5-H5A } & 0.98 & \text { C5-H5B } & 0.98 \\ \text { C6-H6A } & 0.98 & \text { C6-H6B } & 0.98 \\ \text { C7-C8 } & 1.533(3) & \text { C7-C12 } & 1.540(3) \\ \text { C7-H7 } & 0.99 & \text { C8-C9 } & 1.523(3) \\ \text { C8-H8A } & 0.98 & \text { C8-H8B } & 0.98 \\ \text { C9-C10 } & 1.520(4) & \text { C9-H9A } & 0.98 \\ \text { C9-H9B } & 0.98 & \text { C10-C11 } & 1.519(4) \\ \text { C10-H10A } & 0.98 & \text { C10-H10B } & 0.98 \\ \text { C11-C12 } & 1.523(3) & \text { C11-H11A } & 0.98 \\ \text { C11-H11B } & 0.98 & \text { C12-H12A } & 0.98 \\ \text { C12-H12B } & 0.98 & \text { C13-C18 } & 1.536(3) \\ \text { C13-C14 } & 1.538(3) & \text { C13-H13 } & 0.99 \\ \text { C14-C15 } & 1.520(3) & \text { C14-H14A } & 0.98 \\ & & & \end{array}$




$\begin{array}{llll}\text { C14-H14B } & 0.98 & \text { C15-C16 } & 1.519(4) \\ \text { C15-H15A } & 0.98 & \text { C15-H15B } & 0.98 \\ \text { C16-C17 } & 1.511(4) & \text { C16-H16A } & 0.98 \\ \text { C16-H16B } & 0.98 & \text { C17-C18 } & 1.527(3) \\ \text { C17-H17A } & 0.98 & \text { C17-H17B } & 0.98 \\ \text { C18-H18A } & 0.98 & \text { C18-H18B } & 0.98 \\ \text { C19-H19A } & 0.97 & \text { C19-H19B } & 0.97 \\ \text { C19-H19C } & 0.97 & \text { C20-H20A } & 0.97 \\ \text { C20-H20B } & 0.97 & \text { C20-H20C } & 0.97 \\ \text { C21-C22 } & 1.521(4) & \text { C21-H21A } & 0.98 \\ \text { C21-H21B } & 0.98 & \text { C22-H22A } & 0.97 \\ \text { C22-H22B } & 0.97 & \text { C22-H22C } & 0.97 \\ \text { F1-C24 } & 1.351(3) & \text { F2-C25 } & 1.348(3) \\ \text { F3-C26 } & 1.344(3) & \text { F4-C27 } & 1.349(3) \\ \text { F5-C28 } & 1.354(3) & \text { F6-C30 } & 1.346(3) \\ \text { F7-C31 } & 1.345(3) & \text { F8-C32 } & 1.351(3) \\ \text { F9-C33 } & 1.344(3) & \text { F10-C34 } & 1.358(3) \\ \text { F11-C36 } & 1.354(2) & \text { F12-C37 } & 1.348(2) \\ \text { F13-C38 } & 1.340(3) & \text { F14-C39 } & 1.344(3) \\ \text { F15-C40 } & 1.353(2) & \text { F16-C42 } & 1.355(3) \\ \text { F17-C43 } & 1.348(3) & \text { F18-C44 } & 1.341(3) \\ \text { F19-C45 } & 1.348(3) & \text { F20-C46 } & 1.350(3) \\ \text { C23-C28 } & 1.384(3) & \text { C23-C24 } & 1.384(3) \\ \text { C23-B1 } & 1.658(3) & \text { C24-C25 } & 1.383(3) \\ \text { C25-C26 } & 1.368(4) & \text { C26-C27 } & 1.372(4) \\ \text { C27-C28 } & 1.378(3) & \text { C29-C34 } & 1.378(3) \\ \text { C29-C30 } & 1.390(3) & \text { C29-B1 } & 1.658(3) \\ \text { C30-C31 } & 1.380(3) & \text { C31-C32 } & 1.366(4) \\ \text { C32-C33 } & 1.370(4) & \text { C33-C34 } & 1.380(4) \\ \text { C35-C40 } & 1.389(3) & \text { C35-C36 } & 1.390(3) \\ \text { C35-B1 } & 1.665(3) & \text { C36-C37 } & 1.378(3) \\ \text { C37-C38 } & 1.365(3) & \text { C38-C39 } & 1.381(3) \\ \text { C39-C40 } & 1.374(3) & \text { C41-C42 } & 1.388(3) \\ \text { C41-C46 } & 1.389(3) & \text { C41-B1 } & 1.653(3) \\ \text { C42-C43 } & 1.372(3) & \text { C43-C44 } & 1.375(4) \\ \text { C44-C45 } & 1.362(4) & \text { C45-C46 } & 1.379(3) \\ & & & \end{array}$

Table S9. Bond angles $\left({ }^{\circ}\right)$ for rds932.

$\begin{array}{llll}\text { C13-P1-C7 } & 111.77(10) & \text { C13-P1-C1 } & 111.58(10) \\ \text { C7-P1-C1 } & 112.68(10) & \text { C13-P1-Si1 } & 107.11(8) \\ \text { C7-P1-Si1 } & 106.45(7) & \text { C1-P1-Si1 } & 106.83(7) \\ \text { C19-Si1-C20 } & 111.74(13) & \text { C19-Si1-C21 } & 111.36(13)\end{array}$




\begin{tabular}{|c|c|c|c|}
\hline C20-Si1-C21 & $111.44(13)$ & C19-Si1-P1 & 107.03(9) \\
\hline C20-Si1-P1 & $107.20(9)$ & C21-Si1-P1 & $107.80(9)$ \\
\hline C6-C1-C2 & $111.40(18)$ & C6-C1-P1 & $118.08(15)$ \\
\hline C2-C1-P1 & $109.96(15)$ & $\mathrm{C} 6-\mathrm{C} 1-\mathrm{H} 1$ & 105.5 \\
\hline $\mathrm{C} 2-\mathrm{C} 1-\mathrm{H} 1$ & 105.5 & $\mathrm{P} 1-\mathrm{C} 1-\mathrm{H} 1$ & 105.5 \\
\hline C3-C2-C1 & $110.66(19)$ & $\mathrm{C} 3-\mathrm{C} 2-\mathrm{H} 2 \mathrm{~A}$ & 109.5 \\
\hline $\mathrm{C} 1-\mathrm{C} 2-\mathrm{H} 2 \mathrm{~A}$ & 109.5 & C3-C2-H2B & 109.5 \\
\hline C1-C2-H2B & 109.5 & $\mathrm{H} 2 \mathrm{~A}-\mathrm{C} 2-\mathrm{H} 2 \mathrm{~B}$ & 108.1 \\
\hline C4-C3-C2 & $111.2(2)$ & C4-C3-H3A & 109.4 \\
\hline C2-C3-H3A & 109.4 & C4-C3-H3B & 109.4 \\
\hline C2-C3-H3B & 109.4 & H3A-C3-H3B & 108 \\
\hline C3-C4-C5 & $111.1(2)$ & C3-C4-H4A & 109.4 \\
\hline C5-C4-H4A & 109.4 & C3-C4-H4B & 109.4 \\
\hline C5-C4-H4B & 109.4 & $\mathrm{H} 4 \mathrm{~A}-\mathrm{C} 4-\mathrm{H} 4 \mathrm{~B}$ & 108 \\
\hline C4-C5-C6 & $111.5(2)$ & C4-C5-H5A & 109.3 \\
\hline C6-C5-H5A & 109.3 & C4-C5-H5B & 109.3 \\
\hline C6-C5-H5B & 109.3 & H5A-C5-H5B & 108 \\
\hline C1-C6-C5 & $109.67(19)$ & C1-C6-H6A & 109.7 \\
\hline C5-C6-H6A & 109.7 & C1-C6-H6B & 109.7 \\
\hline C5-C6-H6B & 109.7 & H6A-C6-H6B & 108.2 \\
\hline C8-C7-C12 & $110.87(17)$ & C8-C7-P1 & $117.54(15)$ \\
\hline C12-C7-P1 & $109.93(15)$ & $\mathrm{C} 8-\mathrm{C} 7-\mathrm{H} 7$ & 105.9 \\
\hline $\mathrm{C} 12-\mathrm{C} 7-\mathrm{H} 7$ & 105.9 & $\mathrm{P} 1-\mathrm{C} 7-\mathrm{H} 7$ & 105.9 \\
\hline C9-C8-C7 & $109.81(19)$ & C9-C8-H8A & 109.7 \\
\hline C7-C8-H8A & 109.7 & C9-C8-H8B & 109.7 \\
\hline C7-C8-H8B & 109.7 & H8A-C8-H8B & 108.2 \\
\hline C10-C9-C8 & $112.2(2)$ & C10-C9-H9A & 109.2 \\
\hline C8-C9-H9A & 109.2 & C10-C9-H9B & 109.2 \\
\hline C8-C9-H9B & 109.2 & H9A-C9-H9B & 107.9 \\
\hline C11-C10-C9 & $111.4(2)$ & C11-C10-H10A & 109.4 \\
\hline $\mathrm{C} 9-\mathrm{C} 10-\mathrm{H} 10 \mathrm{~A}$ & 109.4 & C11-C10-H10B & 109.4 \\
\hline C9-C10-H10B & 109.4 & $\mathrm{H} 10 \mathrm{~A}-\mathrm{C} 10-\mathrm{H} 10 \mathrm{~B}$ & 108 \\
\hline C10-C11-C12 & $111.1(2)$ & C10-C11-H11A & 109.4 \\
\hline $\mathrm{C} 12-\mathrm{C} 11-\mathrm{H} 11 \mathrm{~A}$ & 109.4 & C10-C11-H11B & 109.4 \\
\hline $\mathrm{C} 12-\mathrm{C} 11-\mathrm{H} 11 \mathrm{~B}$ & 109.4 & $\mathrm{H} 11 \mathrm{~A}-\mathrm{C} 11-\mathrm{H} 11 \mathrm{~B}$ & 108 \\
\hline C11-C12-C7 & $110.15(19)$ & C11-C12-H12A & 109.6 \\
\hline $\mathrm{C} 7-\mathrm{C} 12-\mathrm{H} 12 \mathrm{~A}$ & 109.6 & C11-C12-H12B & 109.6 \\
\hline $\mathrm{C} 7-\mathrm{C} 12-\mathrm{H} 12 \mathrm{~B}$ & 109.6 & $\mathrm{H} 12 \mathrm{~A}-\mathrm{C} 12-\mathrm{H} 12 \mathrm{~B}$ & 108.1 \\
\hline C18-C13-C14 & $111.86(18)$ & C18-C13-P1 & $115.98(16)$ \\
\hline C14-C13-P1 & $109.48(15)$ & $\mathrm{C} 18-\mathrm{C} 13-\mathrm{H} 13$ & 106.3 \\
\hline $\mathrm{C} 14-\mathrm{C} 13-\mathrm{H} 13$ & 106.3 & P1-C13-H13 & 106.3 \\
\hline C15-C14-C13 & 111.16(19) & C15-C14-H14A & 109.4 \\
\hline C13-C14-H14A & 109.4 & C15-C14-H14B & 109.4 \\
\hline
\end{tabular}




$\begin{array}{llll}\text { C13-C14-H14B } & 109.4 & \text { H14A-C14-H14B } & 108 \\ \text { C16-C15-C14 } & 111.3(2) & \text { C16-C15-H15A } & 109.4 \\ \text { C14-C15-H15A } & 109.4 & \text { C16-C15-H15B } & 109.4 \\ \text { C14-C15-H15B } & 109.4 & \text { H15A-C15-H15B } & 108 \\ \text { C17-C16-C15 } & 111.2(2) & \text { C17-C16-H16A } & 109.4 \\ \text { C15-C16-H16A } & 109.4 & \text { C17-C16-H16B } & 109.4 \\ \text { C15-C16-H16B } & 109.4 & \text { H16A-C16-H16B } & 108 \\ \text { C16-C17-C18 } & 111.2(2) & \text { C16-C17-H17A } & 109.4 \\ \text { C18-C17-H17A } & 109.4 & \text { C16-C17-H17B } & 109.4 \\ \text { C18-C17-H17B } & 109.4 & \text { H17A-C17-H17B } & 108 \\ \text { C17-C18-C13 } & 110.5(2) & \text { C17-C18-H18A } & 109.5 \\ \text { C13-C18-H18A } & 109.5 & \text { C17-C18-H18B } & 109.5 \\ \text { C13-C18-H18B } & 109.5 & \text { H18A-C18-H18B } & 108.1 \\ \text { Si1-C19-H19A } & 109.5 & \text { Si1-C19-H19B } & 109.5 \\ \text { H19A-C19-H19B } & 109.5 & \text { Si1-C19-H19C } & 109.5 \\ \text { H19A-C19-H19C } & 109.5 & \text { H19B-C19-H19C } & 109.5 \\ \text { Si1-C20-H20A } & 109.5 & \text { Si1-C20-H20B } & 109.5 \\ \text { H20A-C20-H20B } & 109.5 & \text { Si1-C20-H20C } & 109.5 \\ \text { H20A-C20-H20C } & 109.5 & \text { H20B-C20-H20C } & 109.5 \\ \text { C22-C21-Si1 } & 111.9(2) & \text { C22-C21-H21A } & 109.2 \\ \text { Si1-C21-H21A } & 109.2 & \text { C22-C21-H21B } & 109.2 \\ \text { Si1-C21-H21B } & 109.2 & \text { H21A-C21-H21B } & 107.9 \\ \text { C21-C22-H22A } & 109.5 & \text { C21-C22-H22B } & 109.5 \\ \text { H22A-C22-H22B } & 109.5 & \text { C21-C22-H22C } & 109.5 \\ \text { H22A-C22-H22C } & 109.5 & \text { H22B-C22-H22C } & 109.5 \\ \text { C28-C23-C24 } & 113.1(2) & \text { C28-C23-B1 } & 118.92(19) \\ \text { C24-C23-B1 } & 127.91(19) & \text { F1-C24-C25 } & 114.8(2) \\ \text { F1-C24-C23 } & 121.0(2) & \text { C25-C24-C23 } & 124.2(2) \\ \text { F2-C25-C26 } & 120.0(2) & \text { F2-C25-C24 } & 120.2(2) \\ \text { C26-C25-C24 } & 119.7(2) & \text { F3-C26-C25 } & 120.9(2) \\ \text { F3-C26-C27 } & 120.2(2) & \text { C25-C26-C27 } & 118.9(2) \\ \text { F4-C27-C26 } & 119.7(2) & \text { F4-C27-C28 } & 120.9(2) \\ \text { C26-C27-C28 } & 119.4(2) & \text { F5-C28-C27 } & 116.0(2) \\ \text { F5-C28-C23 } & 119.3(2) & \text { C27-C28-C23 } & 124.7(2) \\ \text { C34-C29-C30 } & 112.8(2) & \text { C34-C29-B1 } & 128.5(2) \\ \text { C30-C29-B1 } & 118.61(19) & \text { F6-C30-C31 } & 116.2(2) \\ \text { F6-C30-C29 } & 119.4(2) & \text { C31-C30-C29 } & 124.4(2) \\ \text { F7-C31-C32 } & 119.9(2) & \text { F7-C31-C30 } & 120.6(2) \\ \text { C32-C31-C30 } & 119.5(2) & \text { F8-C32-C31 } & 120.4(3) \\ \text { F8-C32-C33 } & 120.6(3) & \text { C31-C32-C33 } & 119.0(2) \\ \text { F9-C33-C32 } & 120.0(2) & \text { F9-C33-C34 } & 120.6(3) \\ \text { C32-C33-C34 } & 119.3(2) & \text { F10-C34-C29 } & 121.1(2) \\ \text { F10-C34-C33 } & 114.2(2) & \text { C29-C34-C33 } & 124.7(2)\end{array}$




$\begin{array}{llll}\text { C40-C35-C36 } & 112.73(19) & \text { C40-C35-B1 } & 119.02(18) \\ \text { C36-C35-B1 } & 127.95(19) & \text { F11-C36-C37 } & 114.51(18) \\ \text { F11-C36-C35 } & 121.21(19) & \text { C37-C36-C35 } & 124.3(2) \\ \text { F12-C37-C38 } & 119.3(2) & \text { F12-C37-C36 } & 120.5(2) \\ \text { C38-C37-C36 } & 120.2(2) & \text { F13-C38-C37 } & 121.3(2) \\ \text { F13-C38-C39 } & 120.2(2) & \text { C37-C38-C39 } & 118.6(2) \\ \text { F14-C39-C40 } & 121.3(2) & \text { F14-C39-C38 } & 119.3(2) \\ \text { C40-C39-C38 } & 119.4(2) & \text { F15-C40-C39 } & 115.7(2) \\ \text { F15-C40-C35 } & 119.42(19) & \text { C39-C40-C35 } & 124.9(2) \\ \text { C42-C41-C46 } & 112.9(2) & \text { C42-C41-B1 } & 120.11(19) \\ \text { C46-C41-B1 } & 126.7(2) & \text { F16-C42-C43 } & 116.2(2) \\ \text { F16-C42-C41 } & 119.0(2) & \text { C43-C42-C41 } & 124.7(2) \\ \text { F17-C43-C42 } & 120.7(2) & \text { F17-C43-C44 } & 120.0(2) \\ \text { C42-C43-C44 } & 119.3(2) & \text { F18-C44-C45 } & 120.9(3) \\ \text { F18-C44-C43 } & 120.2(3) & \text { C45-C44-C43 } & 118.9(2) \\ \text { F19-C45-C44 } & 120.0(2) & \text { F19-C45-C46 } & 120.0(2) \\ \text { C44-C45-C46 } & 120.0(2) & \text { F20-C46-C45 } & 114.9(2) \\ \text { F20-C46-C41 } & 121.1(2) & \text { C45-C46-C41 } & 124.1(2) \\ \text { C41-B1-C23 } & 112.04(17) & \text { C41-B1-C29 } & 112.89(18) \\ \text { C23-B1-C29 } & 103.24(17) & \text { C41-B1-C35 } & 102.41(17) \\ \text { C23-B1-C35 } & 113.79(18) & \text { C29-B1-C35 } & 112.84(17) \\ & & & \end{array}$

Table S10. Torsion angles $\left({ }^{\circ}\right)$ for rds932.

$\begin{array}{llll}\text { C13-P1-C1-C6 } & 47.5(2) & \text { C7-P1-C1-C6 } & -79.18(19) \\ \text { Si1-P1-C1-C6 } & 164.27(15) & \text { C13-P1-C1-C2 } & 176.84(15) \\ \text { C7-P1-C1-C2 } & 50.15(18) & \text { Si1-P1-C1-C2 } & -66.41(15) \\ \text { C6-C1-C2-C3 } & -55.8(2) & \text { P1-C1-C2-C3 } & 171.32(16) \\ \text { C1-C2-C3-C4 } & 55.6(3) & \text { C2-C3-C4-C5 } & -56.3(3) \\ \text { C3-C4-C5-C6 } & 57.2(3) & \text { C2-C1-C6-C5 } & 55.9(2) \\ \text { P1-C1-C6-C5 } & -175.42(16) & \text { C4-C5-C6-C1 } & -56.5(3) \\ \text { C13-P1-C7-C8 } & -75.63(18) & \text { C1-P1-C7-C8 } & 50.96(19) \\ \text { Si1-P1-C7-C8 } & 167.74(15) & \text { C13-P1-C7-C12 } & 52.43(18) \\ \text { C1-P1-C7-C12 } & 179.02(15) & \text { Si1-P1-C7-C12 } & -64.20(16) \\ \text { C12-C7-C8-C9 } & 56.8(3) & \text { P1-C7-C8-C9 } & -175.61(16) \\ \text { C7-C8-C9-C10 } & -55.6(3) & \text { C8-C9-C10-C11 } & 55.2(3) \\ \text { C9-C10-C11-C12 } & -55.5(3) & \text { C10-C11-C12-C7 } & 56.7(3) \\ \text { C8-C7-C12-C11 } & -57.8(3) & \text { P1-C7-C12-C11 } & 170.56(17) \\ \text { C7-P1-C13-C18 } & 52.20(19) & \text { C1-P1-C13-C18 } & -74.99(18) \\ \text { Si1-P1-C13-C18 } & 168.43(14) & \text { C7-P1-C13-C14 } & 179.91(15) \\ \text { C1-P1-C13-C14 } & 52.72(18) & \text { Si1-P1-C13-C14 } & -63.86(16) \\ \text { C18-C13-C14-C15 } & -53.5(3) & \text { P1-C13-C14-C15 } & 176.52(17) \\ \text { C13-C14-C15-C16 } & 54.6(3) & \text { C14-C15-C16-C17 } & -57.0(3)\end{array}$




\begin{tabular}{|c|c|c|c|}
\hline C15-C16-C17-C18 & $57.7(3)$ & C16-C17-C18-C13 & $-55.9(3)$ \\
\hline C14-C13-C18-C17 & $53.9(2)$ & P1-C13-C18-C17 & $-179.61(16)$ \\
\hline C19-Si1-C21-C22 & $-67.9(2)$ & C20-Si1-C21-C22 & $57.6(2)$ \\
\hline P1-Si1-C21-C22 & $175.0(2)$ & C28-C23-C24-F1 & $-177.68(19)$ \\
\hline B1-C23-C24-F1 & $-0.3(3)$ & C28-C23-C24-C25 & $1.7(3)$ \\
\hline B1-C23-C24-C25 & $179.1(2)$ & F1-C24-C25-F2 & $-0.6(3)$ \\
\hline C23-C24-C25-F2 & $179.9(2)$ & F1-C24-C25-C26 & $178.8(2)$ \\
\hline C23-C24-C25-C26 & $-0.7(4)$ & F2-C25-C26-F3 & $-0.3(4)$ \\
\hline C24-C25-C26-F3 & $-179.8(2)$ & F2-C25-C26-C27 & $179.0(2)$ \\
\hline C24-C25-C26-C27 & $-0.4(4)$ & F3-C26-C27-F4 & $-0.8(4)$ \\
\hline C25-C26-C27-F4 & $179.9(2)$ & F3-C26-C27-C28 & $179.6(2)$ \\
\hline C25-C26-C27-C28 & $0.2(4)$ & F4-C27-C28-F5 & $-0.2(3)$ \\
\hline C26-C27-C28-F5 & $179.4(2)$ & F4-C27-C28-C23 & $-178.6(2)$ \\
\hline C26-C27-C28-C23 & $1.0(4)$ & C24-C23-C28-F5 & 179.78(19) \\
\hline B1-C23-C28-F5 & $2.2(3)$ & C24-C23-C28-C27 & $-1.9(3)$ \\
\hline B1-C23-C28-C27 & $-179.5(2)$ & C34-C29-C30-F6 & 177.74(19) \\
\hline B1-C29-C30-F6 & $-0.2(3)$ & C34-C29-C30-C31 & $-4.4(3)$ \\
\hline B1-C29-C30-C31 & $177.7(2)$ & F6-C30-C31-F7 & $0.4(3)$ \\
\hline C29-C30-C31-F7 & $-177.5(2)$ & F6-C30-C31-C32 & $178.5(2)$ \\
\hline C29-C30-C31-C32 & $0.6(4)$ & F7-C31-C32-F8 & $0.8(4)$ \\
\hline C30-C31-C32-F8 & $-177.3(2)$ & F7-C31-C32-C33 & $-178.8(2)$ \\
\hline C30-C31-C32-C33 & $3.1(4)$ & F8-C32-C33-F9 & $-2.5(4)$ \\
\hline C31-C32-C33-F9 & $177.1(2)$ & F8-C32-C33-C34 & $177.7(2)$ \\
\hline C31-C32-C33-C34 & $-2.7(4)$ & C30-C29-C34-F10 & $-174.9(2)$ \\
\hline B1-C29-C34-F10 & $2.8(4)$ & C30-C29-C34-C33 & $4.9(3)$ \\
\hline B1-C29-C34-C33 & $-177.4(2)$ & F9-C33-C34-F10 & $-1.6(3)$ \\
\hline C32-C33-C34-F10 & $178.2(2)$ & F9-C33-C34-C29 & $178.6(2)$ \\
\hline C32-C33-C34-C29 & $-1.6(4)$ & C40-C35-C36-F11 & 178.37(19) \\
\hline B1-C35-C36-F11 & $4.7(3)$ & C40-C35-C36-C37 & $-1.9(3)$ \\
\hline B1-C35-C36-C37 & $-175.5(2)$ & F11-C36-C37-F12 & $1.4(3)$ \\
\hline C35-C36-C37-F12 & $-178.3(2)$ & F11-C36-C37-C38 & $-178.3(2)$ \\
\hline C35-C36-C37-C38 & $1.9(4)$ & F12-C37-C38-F13 & $-1.1(4)$ \\
\hline C36-C37-C38-F13 & $178.6(2)$ & F12-C37-C38-C39 & $179.5(2)$ \\
\hline C36-C37-C38-C39 & $-0.8(4)$ & F13-C38-C39-F14 & $-1.0(4)$ \\
\hline C37-C38-C39-F14 & $178.4(2)$ & F13-C38-C39-C40 & $-179.6(2)$ \\
\hline C37-C38-C39-C40 & $-0.1(4)$ & F14-C39-C40-F15 & $1.4(3)$ \\
\hline C38-C39-C40-F15 & $179.9(2)$ & F14-C39-C40-C35 & $-178.5(2)$ \\
\hline C38-C39-C40-C35 & $0.0(4)$ & C36-C35-C40-F15 & $-178.97(19)$ \\
\hline B1-C35-C40-F15 & $-4.7(3)$ & C36-C35-C40-C39 & $0.9(3)$ \\
\hline B1-C35-C40-C39 & $175.2(2)$ & C46-C41-C42-F16 & $-177.3(2)$ \\
\hline B1-C41-C42-F16 & $-2.7(3)$ & C46-C41-C42-C43 & $3.4(3)$ \\
\hline B1-C41-C42-C43 & $178.0(2)$ & F16-C42-C43-F17 & $0.2(3)$ \\
\hline C41-C42-C43-F17 & $179.6(2)$ & F16-C42-C43-C44 & $179.2(2)$ \\
\hline
\end{tabular}




$\begin{array}{llll}\text { C41-C42-C43-C44 } & -1.4(4) & \text { F17-C43-C44-F18 } & -0.1(4) \\ \text { C42-C43-C44-F18 } & -179.1(2) & \text { F17-C43-C44-C45 } & 178.1(2) \\ \text { C42-C43-C44-C45 } & -0.9(4) & \text { F18-C44-C45-F19 } & 1.0(4) \\ \text { C43-C44-C45-F19 } & -177.3(2) & \text { F18-C44-C45-C46 } & 179.1(2) \\ \text { C43-C44-C45-C46 } & 0.8(4) & \text { F19-C45-C46-F20 } & 0.4(3) \\ \text { C44-C45-C46-F20 } & -177.7(2) & \text { F19-C45-C46-C41 } & 179.6(2) \\ \text { C44-C45-C46-C41 } & 1.5(4) & \text { C42-C41-C46-F20 } & 175.7(2) \\ \text { B1-C41-C46-F20 } & 1.5(4) & \text { C42-C41-C46-C45 } & -3.4(3) \\ \text { B1-C41-C46-C45 } & -177.6(2) & \text { C42-C41-B1-C23 } & 174.3(2) \\ \text { C46-C41-B1-C23 } & -12.0(3) & \text { C42-C41-B1-C29 } & 58.2(3) \\ \text { C46-C41-B1-C29 } & -128.0(2) & \text { C42-C41-B1-C35 } & -63.4(2) \\ \text { C46-C41-B1-C35 } & 110.4(2) & \text { C28-C23-B1-C41 } & -62.9(3) \\ \text { C24-C23-B1-C41 } & 119.9(2) & \text { C28-C23-B1-C29 } & 58.8(2) \\ \text { C24-C23-B1-C29 } & -118.4(2) & \text { C28-C23-B1-C35 } & -178.51(19) \\ \text { C24-C23-B1-C35 } & 4.3(3) & \text { C34-C29-B1-C41 } & 5.8(3) \\ \text { C30-C29-B1-C41 } & -176.59(19) & \text { C34-C29-B1-C23 } & -115.3(2) \\ \text { C30-C29-B1-C23 } & 62.2(2) & \text { C34-C29-B1-C35 } & 121.4(2) \\ \text { C30-C29-B1-C35 } & -61.1(3) & \text { C40-C35-B1-C41 } & -58.6(2) \\ \text { C36-C35-B1-C41 } & 114.8(2) & \text { C40-C35-B1-C23 } & 62.6(3) \\ \text { C36-C35-B1-C23 } & -124.1(2) & \text { C40-C35-B1-C29 } & 179.79(19)\end{array}$

Table S11. Anisotropic atomic displacement parameters $\left(\AA^{2}\right)$ for $\mathbf{r d s} 932$.

The anisotropic atomic displacement factor exponent takes the form: $-2 \pi^{2}\left[h^{2} a^{* 2} U_{11}+\ldots+2 h k a^{*} b^{*} U_{12}\right]$

$\begin{array}{lllllll} & U_{11} & U_{22} & U_{33} & U_{23} & U_{13} & \bigcup_{12} \\ \text { P1 } & 0.0157(2) & 0.0204(3) & 0.0216(3) & -0.0003(2) & -0.00305(19) & 0.0000(2) \\ \text { Si1 } & 0.0269(3) & 0.0244(3) & 0.0277(3) & -0.0045(3) & -0.0055(2) & 0.0006(2) \\ \text { C1 } & 0.0178(10) & 0.0259(11) & 0.0253(11) & 0.0021(9) & -0.0031(8) & 0.0019(8) \\ \text { C2 } & 0.0251(11) & 0.0299(11) & 0.0254(12) & -0.0001(9) & -0.0031(9) & 0.0027(9) \\ \text { C3 } & 0.0303(12) & 0.0416(14) & 0.0319(13) & 0.0075(11) & -0.0064(10) & 0.0069(11) \\ \text { C4 } & 0.0354(14) & 0.0422(15) & 0.0422(15) & 0.0089(12) & 0.0001(11) & 0.0160(11) \\ \text { C5 } & 0.0272(12) & 0.0464(15) & 0.0346(14) & 0.0022(11) & 0.0043(10) & 0.0166(11) \\ \text { C6 } & 0.0262(11) & 0.0345(13) & 0.0270(12) & -0.0010(10) & -0.0020(9) & 0.0062(9) \\ \text { C7 } & 0.0189(10) & 0.0242(10) & 0.0217(11) & 0.0010(9) & -0.0003(8) & -0.0007(8) \\ \text { C8 } & 0.0259(11) & 0.0259(11) & 0.0302(13) & -0.0025(9) & 0.0023(9) & -0.0037(9) \\ \text { C9 } & 0.0301(12) & 0.0288(12) & 0.0497(16) & -0.0041(11) & 0.0045(11) & -0.0084(10) \\ \text { C10 } & 0.0251(12) & 0.0432(15) & 0.0567(18) & -0.0006(13) & -0.0015(11) & -0.0118(11) \\ \text { C11 } & 0.0191(11) & 0.0389(14) & 0.0521(16) & 0.0024(12) & 0.0027(10) & 0.0017(10) \\ \text { C12 } & 0.0196(10) & 0.0276(12) & 0.0405(14) & -0.0003(10) & -0.0033(9) & 0.0024(9) \\ \text { C13 } & 0.0184(10) & 0.0279(11) & 0.0243(11) & 0.0039(9) & -0.0019(8) & 0.0027(8) \\ \text { C14 } & 0.0268(11) & 0.0272(11) & 0.0308(13) & -0.0007(10) & 0.0009(9) & -0.0034(9) \\ \text { C15 } & 0.0432(15) & 0.0360(14) & 0.0381(15) & 0.0109(11) & 0.0064(11) & -0.0041(11) \\ \text { C16 } & 0.0355(13) & 0.0571(17) & 0.0273(13) & 0.0087(12) & 0.0042(10) & -0.0024(12) \\ \text { C17 } & 0.0294(12) & 0.0591(17) & 0.0245(13) & 0.0050(12) & -0.0048(10) & -0.0024(12)\end{array}$




\begin{tabular}{|c|c|c|c|c|c|c|}
\hline C18 & $0.0278(12)$ & $0.0409(14)$ & $0.0267(12)$ & $0.0016(10)$ & $-0.0056(9)$ & $-0.0069(10)$ \\
\hline C19 & $0.0352(14)$ & $0.0376(14)$ & $0.0487(16)$ & $-0.0098(12)$ & $-0.0127(12)$ & $-0.0051(11)$ \\
\hline $\mathrm{C} 20$ & $0.0436(15)$ & $0.0282(12)$ & $0.0417(15)$ & $-0.0050(11)$ & $-0.0080(12)$ & $0.0071(11)$ \\
\hline C21 & $0.0472(15)$ & $0.0400(14)$ & $0.0318(14)$ & $-0.0059(11)$ & $0.0053(11)$ & $0.0048(12)$ \\
\hline $\mathrm{C} 22$ & $0.085(2)$ & $0.061(2)$ & $0.0360(17)$ & $-0.0098(15)$ & $0.0020(16)$ & $0.0144(18)$ \\
\hline $\mathrm{F} 1$ & $0.0268(7)$ & $0.0508(9)$ & $0.0339(8)$ & $-0.0058(7)$ & $0.0082(6)$ & $-0.0037(6)$ \\
\hline $\mathrm{F} 2$ & $0.0521(10)$ & $0.0705(11)$ & $0.0277(8)$ & $-0.0089(8)$ & $0.0080(7)$ & $0.0075(8)$ \\
\hline F3 & $0.0611(11)$ & $0.0624(11)$ & $0.0371(9)$ & $-0.0217(8)$ & $-0.0168(8)$ & $0.0023(9)$ \\
\hline $\mathrm{F} 4$ & $0.0376(8)$ & $0.0489(9)$ & $0.0595(11)$ & $-0.0130(8)$ & $-0.0108(7)$ & $-0.0139(7)$ \\
\hline F5 & $0.0231(7)$ & $0.0471(8)$ & $0.0370(8)$ & $-0.0035(7)$ & $0.0034(6)$ & $-0.0071(6)$ \\
\hline F6 & $0.0279(7)$ & $0.0379(8)$ & $0.0408(8)$ & $-0.0021(6)$ & $0.0034(6)$ & $0.0047(6)$ \\
\hline F7 & $0.0528(10)$ & $0.0335(8)$ & $0.0720(12)$ & $0.0025(8)$ & $-0.0200(9)$ & $0.0121(7)$ \\
\hline F8 & $0.0822(13)$ & $0.0438(9)$ & $0.0544(11)$ & $0.0268(8)$ & $-0.0273(9)$ & $-0.0169(9)$ \\
\hline F9 & $0.0725(12)$ & $0.0757(13)$ & $0.0395(10)$ & $0.0201(9)$ & $0.0129(9)$ & $-0.0179(10)$ \\
\hline F10 & $0.0356(8)$ & $0.0525(9)$ & $0.0419(9)$ & $0.0070(7)$ & $0.0166(7)$ & $0.0012(7)$ \\
\hline $\mathrm{F} 11$ & $0.0337(7)$ & $0.0370(7)$ & $0.0312(8)$ & $0.0099(6)$ & $-0.0132(6)$ & $-0.0077(6)$ \\
\hline $\mathrm{F} 12$ & $0.0304(7)$ & $0.0547(9)$ & $0.0404(9)$ & $0.0052(7)$ & $-0.0153(6)$ & $-0.0112(7)$ \\
\hline $\mathrm{F} 13$ & $0.0366(8)$ & $0.0491(9)$ & $0.0548(10)$ & $0.0064(8)$ & $-0.0009(7)$ & $-0.0212(7)$ \\
\hline F14 & $0.0487(9)$ & $0.0418(9)$ & $0.0462(9)$ & $0.0200(7)$ & $-0.0033(7)$ & $-0.0103(7)$ \\
\hline F15 & $0.0285(7)$ & $0.0438(8)$ & $0.0331(8)$ & $0.0116(6)$ & $-0.0084(6)$ & $0.0014(6)$ \\
\hline F16 & $0.0397(8)$ & $0.0521(9)$ & $0.0259(8)$ & $-0.0057(7)$ & $-0.0067(6)$ & $-0.0014(7)$ \\
\hline F17 & $0.0764(12)$ & $0.0514(10)$ & $0.0401(9)$ & $-0.0197(8)$ & $0.0169(8)$ & $-0.0009(9)$ \\
\hline F18 & $0.0531(11)$ & $0.0489(10)$ & $0.0895(14)$ & $-0.0200(10)$ & $0.0194(10)$ & $0.0214(8)$ \\
\hline F19 & $0.0358(8)$ & $0.0520(10)$ & $0.0751(12)$ & $-0.0061(9)$ & $-0.0166(8)$ & $0.0208(7)$ \\
\hline F20 & $0.0312(7)$ & $0.0421(8)$ & $0.0357(8)$ & $-0.0080(6)$ & $-0.0140(6)$ & $0.0092(6)$ \\
\hline C23 & $0.0187(10)$ & $0.0261(11)$ & $0.0259(12)$ & $-0.0009(9)$ & $-0.0040(8)$ & $0.0053(8)$ \\
\hline C24 & $0.0229(11)$ & $0.0307(12)$ & $0.0270(12)$ & $-0.0008(9)$ & $-0.0012(9)$ & $0.0042(9)$ \\
\hline $\mathrm{C} 25$ & $0.0344(13)$ & $0.0419(14)$ & $0.0234(12)$ & $-0.0019(10)$ & $-0.0017(10)$ & $0.0110(11)$ \\
\hline C26 & $0.0420(14)$ & $0.0367(13)$ & $0.0253(13)$ & $-0.0095(10)$ & $-0.0131(10)$ & $0.0086(11)$ \\
\hline $\mathrm{C} 27$ & $0.0262(11)$ & $0.0311(12)$ & $0.0388(14)$ & $-0.0045(10)$ & $-0.0106(10)$ & $-0.0003(10)$ \\
\hline C28 & $0.0221(10)$ & $0.0323(12)$ & $0.0285(12)$ & $-0.0021(10)$ & $-0.0023(9)$ & $0.0029(9)$ \\
\hline C29 & $0.0212(10)$ & $0.0284(11)$ & $0.0253(12)$ & $0.0012(9)$ & $-0.0035(9)$ & $-0.0033(9)$ \\
\hline C30 & $0.0226(11)$ & $0.0289(11)$ & $0.0313(12)$ & $0.0003(10)$ & $-0.0062(9)$ & $-0.0042(9)$ \\
\hline C31 & $0.0349(13)$ & $0.0269(12)$ & $0.0432(15)$ & $0.0011(11)$ & $-0.0161(11)$ & $-0.0035(10)$ \\
\hline C32 & $0.0497(16)$ & $0.0322(13)$ & $0.0389(15)$ & $0.0146(11)$ & $-0.0199(12)$ & $-0.0157(12)$ \\
\hline C33 & $0.0426(15)$ & $0.0455(15)$ & $0.0288(14)$ & $0.0084(11)$ & $-0.0018(11)$ & $-0.0147(12)$ \\
\hline C34 & $0.0289(12)$ & $0.0376(13)$ & $0.0298(13)$ & $0.0015(10)$ & $-0.0003(10)$ & $-0.0045(10)$ \\
\hline C35 & $0.0196(10)$ & $0.0255(11)$ & $0.0215(11)$ & $-0.0024(9)$ & $0.0009(8)$ & $0.0011(8)$ \\
\hline C36 & $0.0258(11)$ & $0.0255(11)$ & $0.0192(11)$ & $0.0014(9)$ & $-0.0012(8)$ & $0.0003(9)$ \\
\hline C37 & $0.0219(11)$ & $0.0352(13)$ & $0.0261(12)$ & $-0.0045(10)$ & $-0.0054(9)$ & $-0.0014(9)$ \\
\hline C38 & $0.0273(11)$ & $0.0311(12)$ & $0.0319(13)$ & $-0.0018(10)$ & $0.0044(9)$ & $-0.0073(9)$ \\
\hline C39 & $0.0326(12)$ & $0.0269(11)$ & $0.0268(12)$ & $0.0044(9)$ & $0.0026(9)$ & $-0.0001(9)$ \\
\hline C40 & $0.0206(10)$ & $0.0307(11)$ & $0.0262(12)$ & $0.0003(9)$ & $-0.0028(9)$ & $0.0027(9)$ \\
\hline C41 & $0.0191(10)$ & $0.0271(11)$ & $0.0260(12)$ & $-0.0004(9)$ & $0.0012(8)$ & $-0.0010(8)$ \\
\hline C42 & $0.0265(11)$ & $0.0345(13)$ & $0.0261(12)$ & $-0.0011(10)$ & $0.0014(9)$ & $-0.0032(9)$ \\
\hline
\end{tabular}




$\begin{array}{lllllll}\text { C43 } & 0.0428(14) & 0.0355(13) & 0.0345(14) & -0.0100(11) & 0.0149(11) & -0.0059(11) \\ \text { C44 } & 0.0338(13) & 0.0310(13) & 0.0575(18) & -0.0072(12) & 0.0134(12) & 0.0065(11) \\ \text { C45 } & 0.0227(11) & 0.0323(13) & 0.0507(16) & -0.0007(12) & -0.0013(10) & 0.0038(10) \\ \text { C46 } & 0.0223(11) & 0.0290(11) & 0.0316(13) & -0.0029(10) & -0.0025(9) & 0.0011(9) \\ \text { B1 } & 0.0176(11) & 0.0294(12) & 0.0194(12) & -0.0010(10) & -0.0009(9) & -0.0002(9)\end{array}$

Table S12. Hydrogen atomic coordinates and isotropic atomic displacement parameters $\left(\AA^{2}\right)$ for rds932.

\begin{tabular}{|c|c|c|c|c|}
\hline & & & & \\
\hline $\mathrm{H} 1$ & 0.4221 & 0.3788 & 0.409 & 0.028 \\
\hline $\mathrm{H} 2 \mathrm{~A}$ & 0.5431 & 0.4401 & 0.2966 & 0.032 \\
\hline $\mathrm{H} 2 \mathrm{~B}$ & 0.5056 & 0.3994 & 0.2395 & 0.032 \\
\hline H3A & 0.3659 & 0.4499 & 0.1933 & 0.042 \\
\hline H3B & 0.3055 & 0.4146 & 0.2583 & 0.042 \\
\hline $\mathrm{H} 4 \mathrm{~A}$ & 0.2436 & 0.474 & 0.3362 & 0.048 \\
\hline $\mathrm{H} 4 \mathrm{~B}$ & 0.376 & 0.4874 & 0.3588 & 0.048 \\
\hline $\mathrm{H} 5 \mathrm{~A}$ & 0.2646 & 0.4234 & 0.4708 & 0.043 \\
\hline H5B & 0.2988 & 0.4642 & 0.5289 & 0.043 \\
\hline $\mathrm{H} 6 \mathrm{~A}$ & 0.5008 & 0.4498 & 0.5135 & 0.035 \\
\hline $\mathrm{H} 6 \mathrm{~B}$ & 0.44 & 0.4145 & 0.5785 & 0.035 \\
\hline $\mathrm{H} 7$ & 0.7507 & 0.4075 & 0.3287 & 0.026 \\
\hline H8A & 0.6897 & 0.4588 & 0.4476 & 0.033 \\
\hline H8B & 0.7652 & 0.4406 & 0.5501 & 0.033 \\
\hline H9A & 0.8597 & 0.4706 & 0.3454 & 0.043 \\
\hline H9B & 0.8758 & 0.4906 & 0.4663 & 0.043 \\
\hline $\mathrm{H} 10 \mathrm{~A}$ & 1.0479 & 0.4558 & 0.4163 & 0.05 \\
\hline H10B & 0.9961 & 0.4389 & 0.5301 & 0.05 \\
\hline $\mathrm{H} 11 \mathrm{~A}$ & 1.044 & 0.3857 & 0.4143 & 0.044 \\
\hline $\mathrm{H} 11 \mathrm{~B}$ & 0.9668 & 0.4033 & 0.3122 & 0.044 \\
\hline $\mathrm{H} 12 \mathrm{~A}$ & 0.8742 & 0.3739 & 0.5185 & 0.035 \\
\hline $\mathrm{H} 12 \mathrm{~B}$ & 0.8596 & 0.3528 & 0.3986 & 0.035 \\
\hline $\mathrm{H} 13$ & 0.6969 & 0.332 & 0.5827 & 0.028 \\
\hline $\mathrm{H} 14 \mathrm{~A}$ & 0.4494 & 0.3431 & 0.597 & 0.034 \\
\hline H14B & 0.5106 & 0.3062 & 0.5407 & 0.034 \\
\hline $\mathrm{H} 15 \mathrm{~A}$ & 0.4507 & 0.2915 & 0.728 & 0.047 \\
\hline H15B & 0.5883 & 0.2857 & 0.716 & 0.047 \\
\hline $\mathrm{H} 16 \mathrm{~A}$ & 0.4808 & 0.353 & 0.8151 & 0.048 \\
\hline $\mathrm{H} 16 \mathrm{~B}$ & 0.5538 & 0.3205 & 0.8837 & 0.048 \\
\hline $\mathrm{H} 17 \mathrm{~A}$ & 0.7266 & 0.3398 & 0.7953 & 0.045 \\
\hline H17B & 0.6705 & 0.3769 & 0.8554 & 0.045 \\
\hline $\mathrm{H} 18 \mathrm{~A}$ & 0.7296 & 0.3932 & 0.6689 & 0.038 \\
\hline H18B & 0.5923 & 0.3998 & 0.6812 & 0.038 \\
\hline
\end{tabular}




$\begin{array}{lrrrr}\text { H19A } & 0.4279 & 0.3299 & 0.2444 & 0.061 \\ \text { H19B } & 0.4296 & 0.3003 & 0.3484 & 0.061 \\ \text { H19C } & 0.465 & 0.2853 & 0.2266 & 0.061 \\ \text { H20A } & 0.6683 & 0.2723 & 0.4516 & 0.057 \\ \text { H20B } & 0.7864 & 0.287 & 0.397 & 0.057 \\ \text { H20C } & 0.7045 & 0.2563 & 0.3311 & 0.057 \\ \text { H21A } & 0.6536 & 0.3595 & 0.1464 & 0.048 \\ \text { H21B } & 0.7772 & 0.3488 & 0.2038 & 0.048 \\ \text { H22A } & 0.7642 & 0.2845 & 0.1295 & 0.091 \\ \text { H22B } & 0.752 & 0.3154 & 0.0291 & 0.091 \\ \text { H22C } & 0.6386 & 0.2941 & 0.075 & 0.091\end{array}$




\section{References}

(1) Lambert, J. B.; Zhang, S.; Ciro, S. M. Silyl Cations in the Solid and in Solution. Organometallics 1994, 13, 2430-2443.

(2) Holthausen, M. H.; Bayne, J. M.; Mallov, I.; Dobrovetsky, R.; Stephan, D. W. 1,2Diphosphonium Dication: A Strong P-Based Lewis Acid in Frustrated Lewis Pair (FLP)-Activations of B-H, Si-H, C-H, and H-H Bonds. J. Am. Chem. Soc. 2015, 137, 7298-7301.

(3) Tolman, C. A. Steric Effects of Phosphorus Ligands in Organometallic Chemistry and Homogeneous Catalysis. Chem. Rev. 1977, 77, 313-348.

(4) Binh, D. H.; Milovanović, M.; Puertes-Mico, J.; Hamdaoui, M.; Zarić, S. D.; Djukic, J.-P. Is the R3 Si Moiety in Metal-Silyl Complexes a Z Ligand? An Answer from the Interaction Energy. Chem. - A Eur. J. 2017, 23, 17058-17069. 